\title{
Fatigue Crack Propagation Along Polymer-Metal Interfaces in Microelectronic Packages
}

by

John S. Guzek

S.B., Materials Science and Engineering Massachusetts Institute of Technology

(June 1995)

Submitted to the Department of Materials Science and Engineering in Partial Fulfillment of the Requirements for the Degree of

MASTER OF SCIENCE IN MATERIALS SCIENCE AND ENGINEERING

at the

Massachusetts Institute of Technology

June 1996

(C) 1996 Massachusetts Institute of Technology

All rights reserved

Signature of author Department of Materials Science and Engineering
May 10, 1996

Certified by Subra Suresh R. P. Simmons Professor of Materials Science and Engineering Thesis Supervisor Accepted by 


\title{
Fatigue Crack Propagation Along Polymer-Metal Interfaces in Microelectronic Packages
}

by

\author{
John S. Guzek
}

Submitted to the Department of Materials Science and Engineering on May 10, 1996, in partial fulfillment of the requirements for the degree of

Master of Science in Materials Science and Engineering

\begin{abstract}
Delamination and cracking at polymer-metal interfaces is a significant problem in microelectronic packaging structures. In service, these materials are generally subjected to cyclic thermo-mechanical loadings. Therefore, the integrity of these polymer-metal interfaces under cyclic loads is of critical importance. Although much work has been done to characterize the static fracture toughness of these interfaces, little has been done to investigate the fatigue crack propagation (FCP) resistance of polymer-metal interfaces commonly found in microelectronic packages.
\end{abstract}

In this study, a fracture mechanics-based technique was used for characterizing fatigue crack propagation at polymer-metal interfaces. Sandwich double-cantilever beam (DCB) specimens were fabricated using nickel and copper-coated copper substrates bonded with a thin layer of silica-filled polymer encapsulant. Under cyclic loading, crack propagation was found to occur at the polymer-metal interface. The interfacial failure mode was verified by SEM analysis of the fatigue fracture surfaces. The crack growth rate was found to have a power-law dependence on the strain energy release rate range, and exhibited a crack growth threshold, much like the fatigue crack growth threshold stress intensity factor range for monolithic bulk metals, polymers, and ceramics. Interfacial FCP data for three candidate encapsulants predicted cracking resistances that were well correlated with package-level reliability tests. By varying the surface roughness of the copper and nickel plating, it was shown that interfacial FCP resistance increased with increasing roughness. The observed increases in FCP resistance were attributed to a reduction in the effective driving force for fatigue fracture along the rougher interfaces.

Thesis Supervisor: Subra Suresh

Title: R. P. Simmons Professor of Materials Science and Engineering 


\section{Acknowledgments}

A project of this magnitude cannot be completed in six months without a good deal of support from others. Therefore, I would like to take a moment to thank all of the people who helped to make my co-op a success.

I wish to express my sincere thanks to my M.I.T. advisor, Professor Subra Suresh. Even though my experimental work was carried out at Intel, Arizona, Prof. Suresh was available for me to contact and provided critical information for defining my experiments. I also appreciated all of the help he gave me upon my return to M.I.T., both with data analysis and the "technical fine-tuning" of my thesis.

Next, I wish to thank my supervisor, Dr. Hamid Azimi, for giving me the opportunity to work on this project. He provided excellent technical insight and guidance, but also gave me freedom in my experimentation and responsibility for providing results, so that I was able to learn on my own, in a very "hands-on" manner. I would also like to thank Dr. Dan Belton for getting management approval and funding for our work.

I would also like to thank the Polymer Core Competency engineers: Peter Brandenburger, Dr. Brad Factor, Amol Kirtikar, Dr. Joan Vrtis, and Dr. Vijay Wakharkar. They all helped to provide a dynamic intellectual environment and were always willing to offer advice and support. All of the technicians whom I worked with deserve a lot of thanks; without them, experiments would never get done! Thanks to Larry, Sue, Martha, Greg, Patrick, and Lars. Special thanks to Cheryl for all her help and hard work with the SEM. I would like to thank my fellow co-ops, Morgan Slade, Kevin Nix, Russell Aoki, and Walter Grayeski, for making my stay at Intel both educational and fun.

Finally, I wish to thank my parents, John and Kathy, and my fiancé, Amy Singer, for their loving support during the pursuit of my college studies.

The experimental work for this thesis was carried out in the summer and fall semester of 1995 at Intel Corporation, Chandler, Arizona, as part of the III-B internship program at MIT. Further analysis of the data was performed and this document was written during the spring term of 1996 at MIT. 


\section{Contents}

1 Introduction 10

1.1 Microelectronic Packages and Interfaces . . . . . . . . . . . 10

1.2 Adhesion . . . . . . . . . . . . . . . 12

1.3 Interfacial Fatigue Crack Propagation $\ldots \ldots \ldots 13$

1.4 Outline of Thesis . . . . . . . . . . . . . . . . . . 14

2 Theoretical Background 15

2.1 Surface Science and Adhesion $\ldots \ldots \ldots \ldots \ldots \ldots$

2.2 Fracture Mechanics . . . . . . . . . . . . . . . . . 18

2.2.1 Energy Balance Approach . . . . . . . . . . . . . 19

2.2.2 Stress Intensity Factor Approach . . . . . . . . . . . . . 20

2.2.3 Modes of Loading . . . . . . . . . . . . . . . . 21

2.2.4 Applications to Adhesive Joints and Interfaces . . . . . . .23

2.3 Fatigue Crack Propagation . . . . . . . . . . . . . . 26

2.3.1 Analysis of Fatigue Crack Propagation Data . . . . . . . . .26

2.3.2 Crack-tip Shielding Mechanisms . . . . . . . . . . . . 28

2.4 Interfacial Fatigue Crack Propagation . . . . . . . . . . . 30

2.4.1 Fatigue Crack Propagation in Adhesive Joints . . . . . . . . 30

2.4.2 Fatigue Crack Propagation at Interfaces . . . . . . . . . . 32

2.4.3 Purpose of this Investigation . . . . . . . . . . . . 33

3 Experimental 35

3.1 Material Systems . . . . . . . . . . . . . . . . . 35

3.1 .1 Substrates . . . . . . . . . . . . . . 35

3.1 .2 Polymer Encapsulants . . . . . . . . . . . . . 36

3.2 Substrate/Polymer Surface Characterization . . . . . . . . . . 37

3.2.1 Contact Angle Goniometry . . . . . . . . . . . . . . . 37

3.2.2 Laser Profilometry . . . . . . . . . . . . . . . 38

3.2 .3 SEM . . . . . . . . . . . . . . . . . 39

3.3 Specimen Preparation . . . . . . . . . . . . . . . . . . . 39

3.4 Fatigue Crack Propagation Experiments . . . . . . . . . . . 42

3.4 .1 Precracking . . . . . . . . . . . . . 42

3.4.2 Fatigue Crack Growth Testing . . . . . . . . . . . . . . . . . . . .

3.4.3 Data analysis . . . . . . . . . . . . . . . . 44

4 Results and Discussion 46

4.1 Surface Energy / Thermodynamic Work of Adhesion . . . . . . . . 46

4.2 Substrate Surface Roughness . . . . . . . . . . . . . . . 49

4.3 Package Level Reliability . . . . . . . . . . . . . . . . . . 56 . . . . . . .

4.4 Fatigue Crack Propagation Experiments . . . . . . . . . . 56

4.4.1 Failure Mode . . . . . . . . . . . . . . . . 57

4.4.2 Encapsulant Comparison . . . . . . . . . . . . . . 59 
4.4.3 Effect of Substrate Plating Metal and Surface Roughness . . 64

4.4.4 Fractography . . . . . . . . . . . . . . . . 67 . . . . . . . . .

4.5 Shielding Mechanisms . . . . . . . . . . . . . 70

4.5.1 Interfacial Crack Deflection . . . . . . . . . . . . . 70

4.5.2 Roughness-induced Closure . . . . . . . . . . . . . . 75

4.5 .3 Asperity Bridging . . . . . . . . . . . . . . . 77

5 Conclusions 79

5.1 Contact Angle Goniometry . . . . . . . . . . . . . . . . . .79

5.2 Interfacial Fatigue Crack Propagation Testing . . . . . . . . . . . 79

5.3 Interfacial Crack-tip Shielding Mechanisms . . . . . . . . . 80

5.4 Recommendations for Future Work . . . . . . . . . . . . . 81

$\begin{array}{lr}\text { Appendix } & 83\end{array}$

$\begin{array}{lr}\text { Bibliography } & 84\end{array}$ 


\section{List of Figures}

Figure 1.1 - Schematic drawing showing the components of a plastic pin grid array (PPGA) package . . . . . . . . . . . . . 11

Figure 2.1 - Separation of two elastic bodies in the calculation of the thermodynamic work of adhesion . . . . . . . . . . 16

Figure 2.2 - The contact angle of a liquid on a solid resulting from the balance of surface and interfacial forces . . . . . . . . . . 16

Figure 2.3 - a) A crack of length $2 a$ in a uniformly stressed, infinite slab,

b) The stresses around a crack tip . . . . . . . . . . . 21

Figure 2.4 - The three modes of loading; a) mode I or pure tensile, b) mode II or in-plane shear, c) mode III or anti-plane shear . . . . . 22

Figure 2.5 - Schematic representation of adhesive and cohesive failure $\ldots \ldots .23$

Figure 2.6 - Schematic drawing of the DCB geometry $\ldots \ldots \ldots \ldots$

Figure 2.7 - A schematic drawing of a typical, logarithmic scaled da/dN vs. $\Delta \mathrm{G}$ plot with three distinct regimes . . . . . . . . . . 27

Figure 2.8 - Illustration of crack tip shielding mechanisms: a) crack deflection; b) roughness induced crack closure; c) crack-bridging by particles; and d) crack-bridging by fibers . . . . . . . . . . . . . . 29

Figure 3.1 - Illustration of the double cantilever beam (DCB) substrate geometry, indicating critical dimensions . . . . . . . . 36

Figure 3.2 - Illustration of the mold used for fabricating DCB specimens . . . . 41

Figure 3.3 - Illustration of typical cure shrinkage, a) without a fillet and

(b) with a fillet . . . . . . . . . . . . . . 41

Figure 3.4 - a) Side view of DCB specimen showing Krak-Gage ${ }^{\circledR}$ location

b) Top-down "cut-away" view of DCB specimen showing the precrack and gage regions . . . . . . . . . . . . . 42

Figure 4.1 - SEM micrographs (1000x) of the nickel plated substrates in the a) as plated and b) blast-treated conditions . . . . . . . . . 51 
Figure 4.2 - SEM micrographs (1000x) of the copper plated substrates in the a) as plated and b) blast-treated conditions . . . . . . . . 5 52

Figure 4.3 - SEM micrographs (1000x) of the encapsulant/nickel interface in specimens made from a) as plated and b) blast-treated substrates . . 53

Figure 4.4 - SEM micrographs (1000x) of the encapsulant/copper interface in specimens made from a) as plated and b) blast-treated substrates . 54

Figure 4.5 - SEM micrographs (150x) of the bonding region of specimens made from a) as plated and b) blast-treated copper substrates . . . . . 55

Figure 4.6 - Graph of crack length vs. time showing that no crack growth occurred during a 30 minute hold at maximum load . . . . . . 57

Figure 4.7 - SEM micrograph (150x) showing the crack path along the top encapsulant/copper interface . . . . . . . . . . . 58

Figure 4.8 - Photograph of typical DCB fracture surfaces $\ldots \ldots \ldots \ldots$. . . 58

Figure 4.9 - Interfacial FCP data for three candidate encapsulants bonded to as-plated nickel . . . . . . . . . . . . . 60

Figure 4.10 - FCP trends for encapsualnt/metal interfaces, bulk encapsulant [3], and bulk metals $[38] \ldots \ldots \ldots$. . . . . . . . . . . 63

Figure 4.11 - Interfacial FCP data for Material B encapsulant bonded to as-plated and blast-treated nickel . . . . . . . . . . . . 65

Figure 4.12 - Interfacial FCP data for Material B encapsulant bonded to as-plated and blast-treated copper . . . . . . . . . . . 66

Figure 4.13 - SEM micrograph (1250x) showing metal fatigue-fracture surface for material B on blasted nickel substrate . . . . . . . . 67

Figure 4.14 - SEM micrograph (1250x) showing encapsulant fatigue-fracture surface for material B on as-plated copper substrate . . . . . . 68

Figure 4.15 - SEM micrograph (1000x) showing encapsulant fatigue-fracture surface for material B on blasted nickel substrate . . . . . . . . 69

Figure 4.16 - SEM micrograph (500x) showing encapsulant fatigue-fracture surface for material $A$ on as-plated nickel substrate . . . . . . .70 
Figure 4.17 - a) Schematic drawing of a crack with periodic tilts, b) Schematic drawing of a deflected crack in fully opened condition at the peak load of the fatigue cycle (on the left) and relative mismatch between the fracture surfaces at the point of first contact during unloading (on the right) . . . . . 72

Figure 4.18 - Predicted FCP rates for deflected cracks as functions of the closure factor $\chi$ for $\theta=45^{\circ}$ and $D / D+S$ values of (a) 0.5 and (b) $0.75 \ldots \ldots .74$

Figure 4.19 - Predicted FCP rates for deflected cracks as functions of the closure factor $\chi$ for $\theta=45^{\circ}$ and $D / D+S=0.75$ along with data for the as-plated and blasttreated copper-encapsulant interfaces . . . . . . . . . . 75

Figure 4.20 - Three conditions for a crack along a faceted interface: a) open everywhere, b) contact with sliding, c) frictional locking . . . . . . . . 76

Figure 4.21 - Illustration of the proposed asperity bridging process $\ldots \ldots \ldots 78$

Figure A.1 - Percent error between $\Delta G$ calculations using the homogenous solution (equation 3.11) and the exact solution (equation 3.12) as a function of dimensionless crack length $(a / W) \ldots \ldots \ldots . \ldots . \ldots 8$ 


\section{List of Tables}

Table 3.1 - Encapsulant chemistry, filler loading, elastic modulus, and recommended cure schedules . . . . . . . . . . . . . . 36

Table 3.2 - Surface energy parameters for the three liquids used in this study [6] .38

Table 4.1 - Pseudo-equilibrium advancing contact angles of the three liquids on metallic substrates and adhesives . . . . . . . . . . 46

Table 4.2 - Total surface energies with dispersive and polar components $\left(\mathrm{mJ} / \mathrm{m}^{2}\right) .48$

Table 4.3 - Work of adhesion $\left(\mathrm{mJ} / \mathrm{m}^{2}\right)$ for adhesives on various substrates . . .49

Table 4.4 - Substrate surface roughness parameters . . . . . . . . . . . 49

Table 4.5 - Delamination results from package-level reliability testing . . . . . 56

Table 4.6 - Summary of key interfacial FCP parameters for candidate encapsulants on asplated nickel . . . . . . . . . . . . . . . . 59

Table 4.7 - Summary of key interfacial FCP parameters for Material B encapsulant on various substrates . . . . . . . . . . . . . . 64 


\section{Chapter 1}

\section{Introduction}

As the products of microelectronics technology move toward greater levels of integration, increasing functionality, and enhanced performance, the demands on microelectronic packaging grow in direct proportion. Once an afterthought in the design process, packaging now plays a fundamental role in allowing products to achieve the levels of performance and reliability that are necessary to take full advantage of today's microelectronics technology. High-performance microprocessor packaging is shifting from the established ceramic-based technology to newer, organic-based designs. This necessary transition brings with it an array of materials related challenges that are critical to the delivery of the next generation of microelectronic products.

\subsection{Microelectronic Packages and Interfaces}

The microelectronic package serves three main functions. First, it provides the means for the integrated circuit (IC) to establish an electrical connection with the rest of the system. Second, it allows the device to function properly by protecting it from the environment. Third, it dissipates the heat generated by the IC while in use. To meet these challenges, modern packages have evolved into complex structures, composed of a wide variety of materials. A schematic cross-sectional view of a typical cavity-down plastic pin-grid array (PPGA) package is shown in Figure 1.1.

The silicon die (IC) is bonded to the heat slug with an organic die-attach adhesive. The bulk of the heat generated by the IC is dissipated through the heat slug. Gold wires connect the die to metal bonding pads on the "shelves" within the cavity. Electrical 
routing layers within the organic laminate package body provide the pathway for the device to communicate with the outside world via the connection pins. A thermoset polymer-based encapsulant is used to fill the cavity and protect the device and wires from the environment.

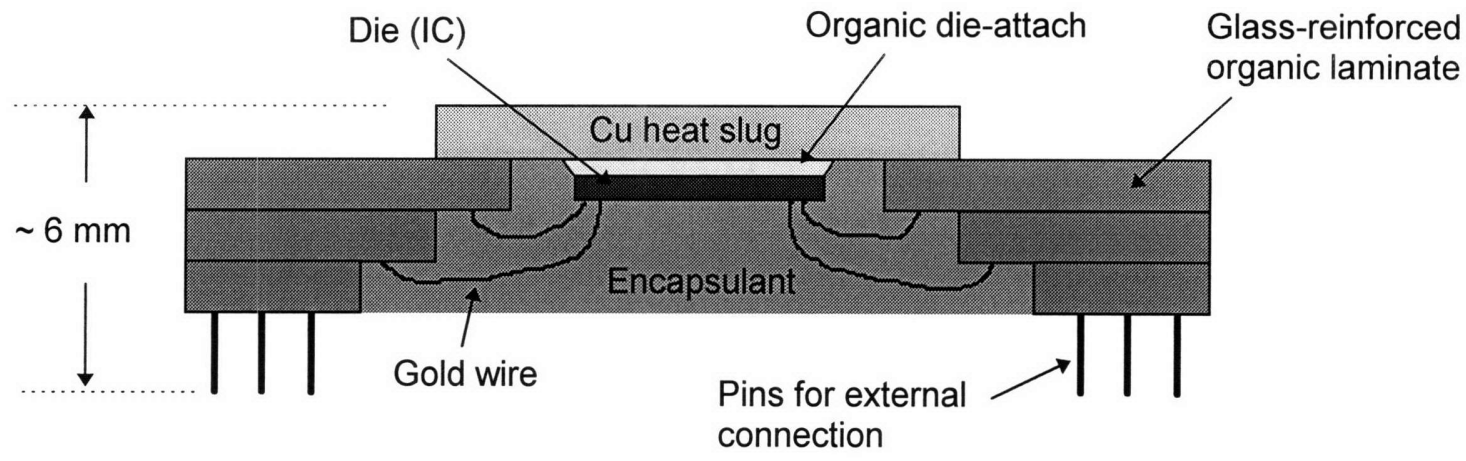

Figure 1.1 - Schematic drawing showing the components of a plastic pin grid array (PPGA) package (note: not drawn to scale)

Before a package can be certified for use, it must pass rigorous environmental (moisture, high temperature) reliability tests ${ }^{1}$. One of the most common types of failures observed after such testing is delamination at an interface. Delamination is the result of the formation and propagation of a crack at an interface, and may originate from internal stresses due to different thermal expansion coefficients between materials, moisture uptake, or defects at the interface. Delamination can occur at any interface, but it is most commonly observed at the encapsulant/heat slug, encapsulant/laminate shelf, and dieattach/heat slug interfaces.

During the development of a new package, it is very costly and time-consuming to build actual packages, subject them to environmental stresses, and then perform failure analysis to determine the extent of delamination. Considering the number of modifications that can be made to either interface material, such full-loop experiments quickly become impractical. Short-loop experiments that mimic the package structure are much more feasible and can significantly aid the development process. Therefore,

\footnotetext{
${ }^{1}$ A typical reliability test called " $85 / 85$ " subjects the package to an environment with a temperature of 85 ${ }^{\circ} \mathrm{C}$ with a relative humidity of $85 \%$.
} 
characterization techniques that quantify interfacial adhesion and correlate to the inservice delamination characteristics of the materials are greatly needed.

\subsection{Adhesion}

Adhesion is a complex phenomenon, and there are several theories regarding its origin. Four main theories have been proposed: mechanical, electronic, adsorption, and diffusion [1]. The mechanical theory states that adhesion arises from the mechanical interlocking of the adhesive material around pores and irregularities in the substrate. According to the electronic theory, materials adhere to one another because of electrostatic forces. In the adsorption theory, the molecules of the adhesive are adsorbed on the substrate and are held in place by secondary bonding (e.g. van der Waals forces, hydrogen bonding, etc.). Finally, the diffusion theory maintains that the adhesive molecules diffuse into the substrate, creating an interfacial zone.

Each theory has its own merits, but no single one has proven to be universally applicable [1]. Even if one mechanism seems to apply to a given situation, the others may also contribute to the adhesive forces acting across the interface. Further confusion can also result from the test methods used to measure adhesion. Typical mechanical tests are not well suited for determining intrinsic adhesive forces. These tests often introduce complicated geometric and loading factors, as well as rheological energy losses in the adhesive and substrates. Some examples of mechanical adhesion tests include the peel test, stud pull, and lap shear [2]. These tests measure a failure load or stress specific to that test and material system. Therefore, comparisons between test methods are difficult, and the adhesive forces are often obscured by other factors inherent to the test method. An adhesive test method is only useful if it can detect changes in the intrinsic interfacial adhesive forces or material properties. Fracture mechanics based test methods that measure fracture toughness $\left(K_{c}\right)$ or fracture energy $\left(G_{c}\right)$ have proven much more useful in this regard.

Typical fracture mechanics based adhesion tests usually involve loading an adhesive specimen until failure occurs. This type of test represents the service environment of a load bearing adhesive joint. However, adhesive interfaces in 
microelectronic packages typically experience cyclic thermomechanical stresses during operation rather than static stresses. Therefore, adhesion under cyclic loading conditions is critical to the microelectronic package performance.

\subsection{Interfacial Fatigue Crack Propagation}

In a recent study on microelectronic package encapsulant materials, it was shown that fatigue crack propagation (FCP) data properly represent the in-service behavior of these materials [3]. FCP testing captures the cracking resistance of the material under cyclic loading conditions. However, besides cracking in encapsulants, delamination and cracking at interfaces is also a critical issue. Although many studies, both experimental and numerical modeling, have addressed adhesion, delamination, and cracking at polymer/metal interfaces found in microelectronic packages [4, 5], we are not aware of any study regarding the interfacial crack growth resistance under cyclic loading conditions. It should, however, be mentioned that quantitative analysis of FCP resistance of adhesive joints for large structural components have been done successfully within the last 10 years, mostly for composite materials $[6,7,8]$ and large scale metal/adhesive bonds $[9,10,11,12]$ for automotive and aerospace applications. Yet, none of these studies focused on interfacial crack growth.

The intention of this study, therefore, is to apply the concepts of FCP testing to polymer/metal interfaces commonly found in microelectronic packaging. The objectives of this study are two-fold. The first objective is to evaluate the feasibility of interfacial fatigue testing as a tool for fatigue life estimation of bi-material interfaces in microelectronic packages. The key result will be the correlation of interfacial FCP resistance observed in laboratory specimens with cracking and delamination in actual package structures. The second objective is to determine the relationships between FCP resistance and the fundamental properties of the constituent materials in the package.

In order to accomplish the first objective, interfacial FCP data on three candidate encapsulant materials were collected, and the results were compared with full-scale, package-level delamination experimental results. The substrates used to simulate the heat slug were manufactured and processed in an identical manner to actual production 
material. The candidate encapsulants differed in basic chemical composition, but were similar in inorganic filler content. Fulfilling the first objective demonstrated the technique's usefulness in materials screening and development.

The second objective was aimed at understanding the mechanisms of cracking and delamination at interfaces. In order to gain insight into the adhesion mechanisms present, both the chemical (type of metal) and physical (surface roughness) properties of the metallic substrate were varied. The interfacial thermodynamic work of adhesion was also determined from contact angle measurements and was compared to the FCP threshold data. The fulfillment of this objective was of interest so that a mechanistic understanding of crack propagation could be applied to the selection and design of future interface materials.

\subsection{Outline of Thesis}

Chapter 2 of this thesis will begin with a discussion of the theoretical aspects of surface science and adhesion. Then, it will examine the theory of fracture mechanics and its applications to the study of adhesive joints and fatigue crack propagation. It will conclude with a discussion of fatigue crack propagation in adhesive joints and other interfacial systems. Chapter 3 will discuss the materials, characterization techniques, and fatigue testing procedure used in this study. The results obtained in this study will be presented and discussed in Chapter 4. Finally, the conclusions which can be drawn from this thesis will be given in Chapter 5 . 


\section{Chapter 2}

\section{Theoretical Background}

\subsection{Surface Science and Adhesion}

The term adhesion generally refers to the physical attraction between two materials. An adhesive system comprises two substrates joined together by an adhesive. Several conditions are necessary to obtain good adhesion [1]. First, the adhesive must wet the substrate in order to provide intimate, interfacial molecular contact. This intimate contact is necessary for the formation of intrinsic adhesive forces between the substrate and adhesive. Second, the adhesive must solidify to withstand the stresses and strains applied to the system and to transmit applied loads. Third, the adhesive must be able to plastically deform in order to dissipate energy at a crack tip. Finally, good adhesion can only be obtained by understanding the joint geometry, the modes in which the loads are applied, and the response of the system to environmental stresses.

One way to examine adhesion is through the surface science approach. This method examines the intrinsic adhesion forces between two surfaces through surface tension measurements. The surface tension is a direct measurement of intermolecular forces, and can be used to determine the thermodynamic work of adhesion, $W^{a}$. The thermodynamic work of adhesion is defined as the Helmholtz free energy change per unit area when separating two elastic bodies (see Figure 2.1). It can be related to the surface and interfacial free energies by the Dupre equation [13]:

$$
W_{12}^{a}=\gamma_{1}+\gamma_{2}-\gamma_{12}
$$


where $\gamma_{1}$ and $\gamma_{2}$ are the surface free energies of the adhesive and the substrate, respectively, and $\gamma_{12}$ is the free energy associated with the interface between the adhesive and the substrate.

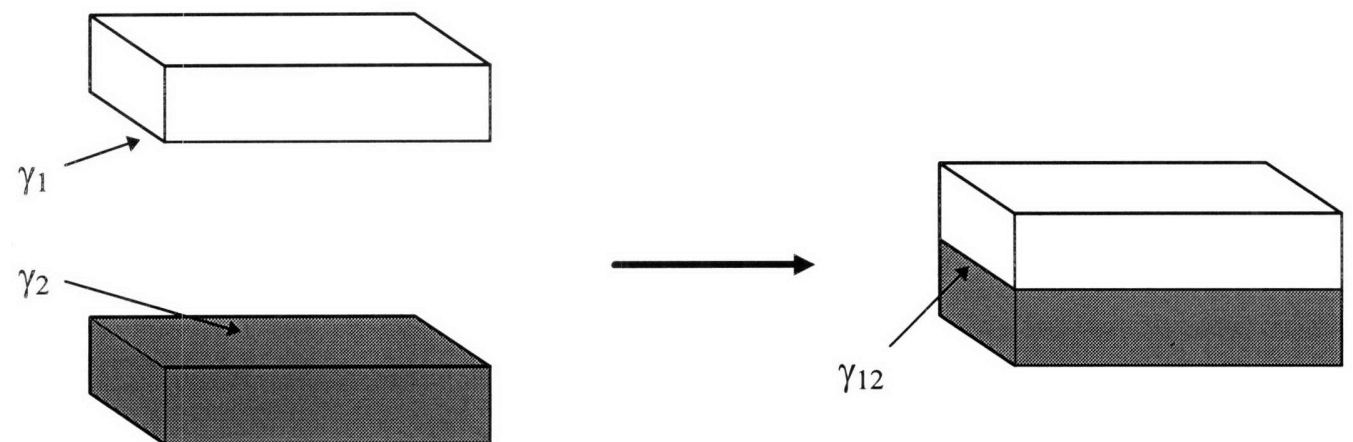

Figure 2.1 - Separation of two elastic bodies in the calculation of the thermodynamic work of adhesion

Surface energy is commonly determined via contact angle measurement techniques. The Young-Dupre equation [13] forms the basis for calculating surface energies from contact angle $(\theta)$ measurements.

$$
\gamma_{L V} \cos (\theta)=\gamma_{S V}-\gamma_{S L}
$$

It represents the balance of surface tension $(\gamma)$ for a liquid drop on a solid substrate, as illustrated in Figure 2.2. The subscripts LV, SV, and SL denote the liquid-vapor, solidvapor, and solid-liquid components of surface tension.

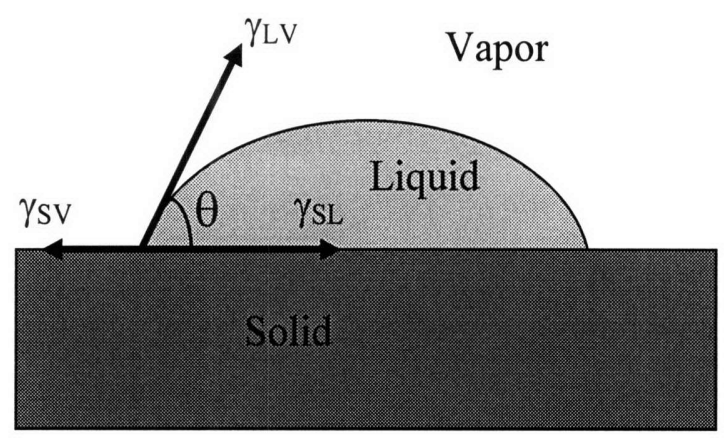

Figure 2.2 - The contact angle of a liquid on a solid resulting from the balance of surface and interfacial forces 
The contact angle can be related to the work of adhesion by combining equation 2.1 and 2.2 .

$$
W_{S L}^{\text {total }}=\gamma_{L}(1+\cos \theta)
$$

The total work of adhesion can also be partitioned into Lewis acid-base and dispersive terms $[13,14]$.

$$
W_{S L}^{\text {total }}=W_{S L}^{d}+W_{S L}^{a b}
$$

The dispersive forces, also known as Lifshitz-van der Waals forces, combine all electromagnetic interactions including oscillating temporary dipoles, permanent dipoles, and induced dipoles [15]. Fowkes [16] has shown that dispersive components of surface energy for the solid and liquid can be calculated via:

$$
W_{S L}^{\text {total }}=2\left(\gamma_{S}^{d} \gamma_{L}^{d}\right)^{\frac{1}{2}}+W_{S L}^{a b} .
$$

The acid-base term of the work of adhesion accounts for interactions between Lewis acids and Lewis bases. Similar solid and liquid surface energy components corresponding to Lewis acid $\left(\gamma^{+}\right)$and Lewis base $\left(\gamma^{-}\right)$contributions can be defined [4], such that

$$
W_{S L}^{\text {total }}=\gamma_{L}(1+\cos \theta)=2 \cdot\left[\left(\gamma_{S}^{d} \gamma_{L}^{d}\right)^{\frac{1}{2}}+\left(\gamma_{S}^{+} \gamma_{L}^{-}\right)^{\frac{1}{2}}+\left(\gamma_{S}^{-} \gamma_{L}^{+}\right)^{\frac{1}{2}}\right] .
$$

Contact angle measurement techniques can be applied to both substrate and cured polymer surfaces to yield the dispersive, Lewis acid, and Lewis base components of surface energy. These components can then be used to calculate the thermodynamic work of adhesion for substrate adhesive pairs:

$$
W_{S A}^{\text {total }}=2 \cdot\left[\left(\gamma_{S}^{d} \gamma_{A}^{d}\right)^{\frac{1}{2}}+\left(\gamma_{S}^{+} \gamma_{A}^{-}\right)^{\frac{1}{2}}+\left(\gamma_{S}^{-} \gamma_{A}^{+}\right)^{\frac{1}{2}}\right]
$$

where the subscripts $\mathrm{S}$ and A now refer to the substrate and adhesive. Thus, a prediction of $W^{a}$ can be made for an adhesive on a substrate in order to compare various systems. Since the adhesives have to be cured against air (not the substrate), the calculated thermodynamic work of adhesion is believed to be less than it would be if it were cured against the substrate [4].

The thermodynamic work of adhesion predicts fracture energy in the $\mathrm{mJ} / \mathrm{m}^{2}$ range while the fracture energy of ductile materials as measured through fracture mechanics 
tests is on the order of $\mathrm{J} / \mathrm{m}^{2}$. This large discrepancy is due to the various energy absorbing processes that occur during fracture aside from the breaking of bonds across the interface. The total energy required to separate two surfaces is referred to as the practical work of adhesion, $W^{P}$. The practical work of adhesion is a measure of the intrinsic adhesion forces (breaking of bonds) as well as the energy dissipated in other manners (e.g. plastic deformation, particle bridging, microcracking, etc.).

Although the thermodynamic work of adhesion is about three orders of magnitude less than $W^{P}$, it can still play an important role in the strength of an interface. Gent and Shultz [17] and Andrews and Kinloch [18] have shown that in elastomers:

$$
W^{P}=W^{A}(1+\Phi)
$$

where $\Phi$ is a parameter that is dependent on the thickness and viscoelastic characteristics of the adhesive, rate of crack propagation, mode of crack propagation, and temperature. Thus, the thermodynamic work of adhesion has a multiplicative effect on the practical work of adhesion. Theoretically, as the magnitude of non-intrinsic energy dissipating processes that occur during fracture decreases, $\Phi$ should approach zero.

While investigating the thermodynamic work of adhesion can provide some useful insights, the strength of an interface ultimately relies on non-intrinsic energy absorbing processes. In reality, an adhesive joint will fail at stresses well below the ultimate strength of the adhesive. This is due to the presence of defects, or cracks, in the bonding area. Therefore, a fracture mechanics approach is employed to test the adhesive properties independent of specimen size or geometry.

\subsection{Fracture Mechanics}

Engineering structures that are designed to operate under stress levels below the yield strength of the structural materials have long been observed to fail by catastrophic fracture. Often, these failures were traced back to the presence of pre-existing cracks. This stimulated the development of the discipline of fracture mechanics with the aim of quantifying the residual strength and service life of materials containing crack-like flaws. Historically, the first approach to obtain a criterion for fracture was based on energy considerations developed by Griffith [19]. Irwin [20] and Orowan [21] later provided an 
equivalent stress analysis approach that was more useful for engineering design purposes. Their approach used the stress state around a crack tip to define a critical material property known as the fracture toughness. A discussion of these two approaches and their applications to adhesive joints follows.

\subsubsection{Energy Balance Approach}

Griffith's hypothesis describes quasi-static crack propagation as the conversion of the work done, $W_{d}$, by the external force and the elastic energy stored in the bulk of the specimen, $U$, into surface free energy, $\gamma_{m}$. It may be written as a failure criterion:

$$
\frac{\partial\left(W_{d}-U\right)}{\partial a} \geq \gamma_{m} \frac{\partial A}{\partial a}
$$

where $\partial A$ is the increase in surface area associated with an increment of crack extension $\partial a$. For a crack propagating in a slab of thickness, $B$, the criterion becomes:

$$
\frac{1}{B} \cdot \frac{\partial\left(W_{d}-U\right)}{\partial a} \geq 2 \gamma_{m} \text {. }
$$

Initial work $[21,22,23]$ conducted on the fracture of metals, crosslinked rubbers, and polymers all found that the energy required to cause crack growth far exceeded twice the interfacial free energy, as predicted by Griffith. There are two main reasons for this discrepancy [1]. First, $2 \gamma_{m}$ only reflects the energy necessary to break secondary bonds, such as Van der Waals forces. Crack growth often involves overcoming stronger forces, such as primary bonds. Secondly, even in the most brittle materials, fracture always involves localized inelastic deformation (such as distributed microcracking) and/or viscoelastic dissipative processes in the highly strained region around the crack tip. These micromechanisms usually represent the main sources of energy absorption during crack growth.

To overcome this limitation, assuming energy dissipation at the crack tip occurs in a manner independent of the test geometry and manner of loading, then $2 \gamma_{m}$ may be replaced in equation 2.10 by the term $G_{c}$. The value $G_{c}$ contains contributions from all energy absorbing processes at the crack tip. It is called the critical strain energy release 
rate, and is equal to the energy required to increase the crack by a unit length in a specimen of unit width. Therefore, the fracture criterion may be rewritten as:

$$
\frac{1}{B} \cdot \frac{\partial\left(W_{d}-U\right)}{\partial a} \geq G_{c} .
$$

\subsubsection{Stress Intensity Factor Approach}

The fracture mechanics method can also be based on stress distributions around the crack tip. For the case of a homogeneous material, Irwin [20] noted that the magnitude of the crack tip stresses depends on the product of the nominal stress, $\sigma$, and the square root of the half flaw length, $a$. This product is the stress intensity factor, $K$ :

$$
K=Y \sigma \sqrt{a}
$$

where $Y$ is a geometric factor which has been computed for various geometries [24]. For the case of a crack of length $2 a$ in a uniformly stressed, infinite slab as shown in Figure 2.3a:

$$
K=\sigma \sqrt{\pi a} .
$$

The stresses around a crack tip as in Figure $2.3 \mathrm{~b}$ are:

$$
\begin{aligned}
& \sigma_{x}=\frac{K}{\sqrt{2 \pi r}}\left[\cos \frac{\theta}{2}\left(1-\sin \frac{\theta}{2} \sin \frac{3 \theta}{2}\right)\right] \\
& \sigma_{y}=\frac{K}{\sqrt{2 \pi r}}\left[\cos \frac{\theta}{2}\left(1+\sin \frac{\theta}{2} \sin \frac{3 \theta}{2}\right)\right] \\
& \tau_{x y}=\frac{K}{\sqrt{2 \pi r}}\left[\sin \frac{\theta}{2} \cos \frac{\theta}{2} \cos \frac{3 \theta}{2}\right]
\end{aligned}
$$

where $r$ is the distance from the crack tip and $\theta$ is the angle to the point from the crack plane as shown in Figure 2.3b. 


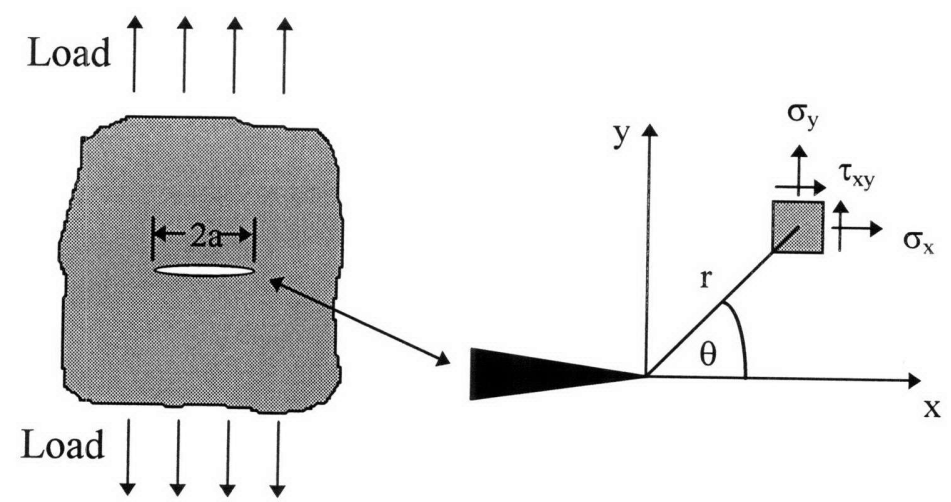

a)

b)

Figure 2.3 - a) A crack of length $2 a$ in a uniformly stressed, infinite slab

b) The stresses around a crack tip

As seen in eq. 2.14, $K$ uniquely describes the crack tip stress field; hence, it can be used as a fracture criterion:

$$
K \geq K_{c}
$$

where $K_{c}$ is a material parameter called the fracture toughness.

Both the stress intensity factor approach and the energy balance approach describe the same phenomenon. Therefore, they can be related through [20]:

$$
G=\frac{K^{2}}{E^{*}}
$$

where $E^{*}=E$ for plane stress conditions and $E^{*}=E /\left(1-v^{2}\right)$ for plane strain conditions, where $E$ is the tensile modulus, and $v$ is the Poisson ratio. It should be noted that this discussion has been based on loading in one direction. In reality, loading can occur in several modes, which will be discussed next.

\subsubsection{Modes of Loading}

A crack can be loaded in three distinct loading modes as shown in Figure 2.4. A crack where the surfaces move directly apart under a tensile load is referred to as pure mode I. Pure mode II is a shear loading where the crack planes slide or undergo in-plane shear. Tearing, or anti-plane shear, is characteristic of mode III loading. In reality, a crack may 
experience any combination of loading modes; therefore, a description of the mode of loading must be reported. The most common mixed mode loading conditions involve a combination of modes I and II. In this case, the fracture toughness may be characterized by the remote stress intensity factors, $K_{I}^{\infty}$ and $K_{I I}{ }^{\infty}$. These stress intensity factors are determined from the applied loads and geometry. From these factors, the remote phase angle can be defined as:

$$
\tan \Psi=\frac{K_{I I}^{\infty}}{K_{I}^{\infty}}
$$

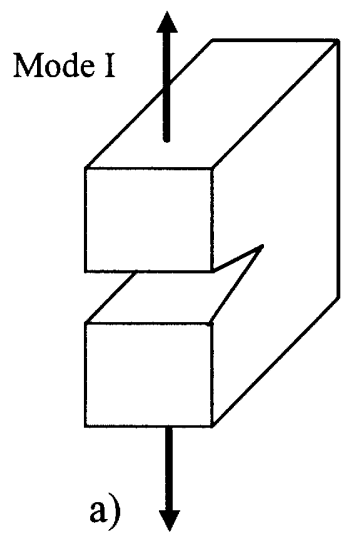

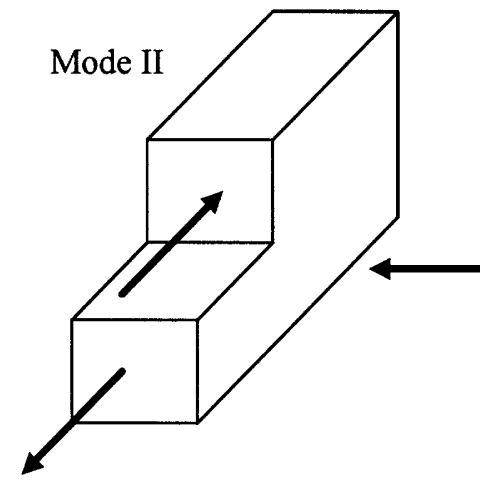

b)

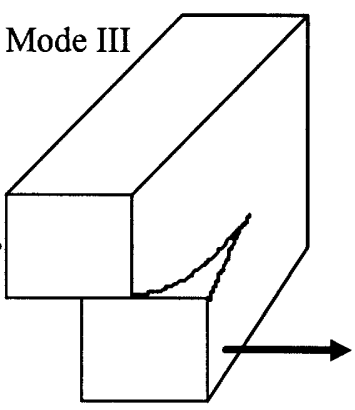

c)

Figure 2.4 - The three modes of loading; a) mode I or pure tensile, b) mode II or in-plane shear, c) mode III or anti-plane shear

The angle $\Psi$ is referred to as the loading mixity or the mixed mode angle of loading.

Like the stress intensity, the critical strain energy release rate, or fracture energy, can also be partitioned into mode I and mode II components. It is important to report the loading mixity, since $G_{c}$ has been found significantly vary with $\Psi$ [25]. When the energy balance approach is used under mixed-mode conditions, the total strain energy release rate $\left(G_{T}\right)$ is partitioned into mode I and mode II components:

$$
G_{T}=G_{I}+G_{I I}
$$

which, for the case of coplanar crack extension, do not interact [26]. Since $G$ is proportional to $K^{2}$, the mixity is also given by: 


$$
\tan \Psi=\sqrt{\frac{G_{I I}}{G_{I}}} .
$$

\subsubsection{Applications to Adhesive Joints and Interfaces}

Adhesive joints are known to fail via the propagation of pre-existing flaws; therefore, fracture mechanics has been widely used to characterize the toughness of adhesive systems [1]. In the study of adhesive joints it is important to distinguish between adhesive and cohesive failure. Crack propagation at the interface between the adhesive and the substrate is referred to as adhesive failure; whereas, cracking through the bulk of the adhesive layer is cohesive failure (see Figure 2.5). Since cohesive fracture is generally dictated by the toughness of the adhesive, not the properties of the interface, it is not considered in this study.

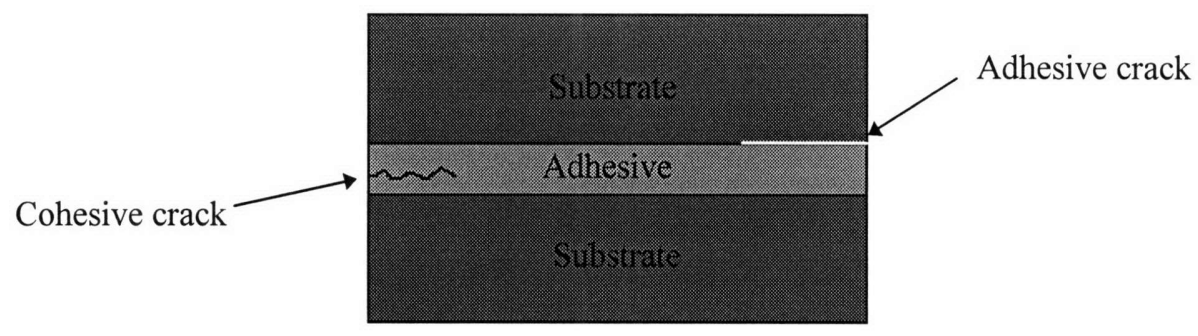

Figure 2.5 - Schematic representation of adhesive and cohesive failure

The case of a crack at an interface between two different materials has received much attention in the literature since the work by Williams [27] in 1959. This intense activity revolves around developing methods to describe the stress state at the crack tip. Due to the inhomogeneity at the bi-material interface, the traditional stress intensity factor cannot be used [28, 29, 30]. Even when the joint is subjected to purely tensile loads applied normal to the crack, the elastic mismatch at the interface will induce both tensile and shear stresses around the crack tip. Much progress has been made in modeling the interfacial crack tip stress state; however, there are still many difficulties and no 
universally accepted models [1]. Therefore, many workers have chosen to adopt the energy balance approach when studying crack growth in adhesive joints.

For an adhesive joint exhibiting bulk linear-elastic behavior, i.e. away from the crack tip region, the strain energy release rate is given by [31]:

$$
G=\frac{P^{2}}{2 B} \frac{\partial C}{\partial a}
$$

where $P$ is the applied force and $C$ is the compliance of the structure and is given by the displacement / load. As long as the adhesive layer is thin compared to the substrate, the compliance of the structure is not affected by the adhesive layer, i.e. it is equivalent to a homogeneous sample made of the substrate material. Equation 2.11 forms the basis for many calculations of $G$. In practice, $C$ is found either experimentally or numerically and is used to determine $\partial C / \partial a$.

A common geometry for adhesion tests is the double cantilever beam (DCB), shown in Figure 2.6. For thin adhesive layers [31]:

$$
\frac{\partial C}{\partial a}=\frac{8}{E_{1} B}\left(\frac{3 a^{2}}{h^{3}}+\frac{1}{h}\right)
$$

where $E_{l}$ is the tensile modulus of the substrate and $B$ and $h$ are the width and height of the substrate beams. Combining equations 2.20 and 2.21, the strain energy release rate as a function of applied load and crack length is given by:

$$
G=\frac{4 P^{2}\left(3 a^{2}+h^{2}\right)}{E_{1} B^{2} h^{3}} .
$$

Since the load is applied normal to the crack faces, the loading mixity of the DCB geometry is, for all practical purposes, close to pure Mode I, i.e. $\Psi \approx 0-5^{\circ}[32,33]$. Therefore, it is often used to calculate the mode I fracture energy,

$$
G_{I C}=\frac{4 P_{c}^{2}\left(3 a^{2}+h^{2}\right)}{E_{1} B^{2} h^{3}}
$$

where $P_{c}$ is the load at the onset of crack propagation. The above equations for the DCB geometry assume a cohesive crack. However, Ozdil and Carlsson [33] performed a numerical analysis on the DCB geometry comparing both cohesive and adhesive cracks. They found that the location of the crack did not noticeably alter the specimen 
compliance or energy release rate as compared to the homogenous case for thin adhesive layers. Therefore, data reduction techniques for the homogenous case were found to be adequate for DCB interfacial fracture specimens.

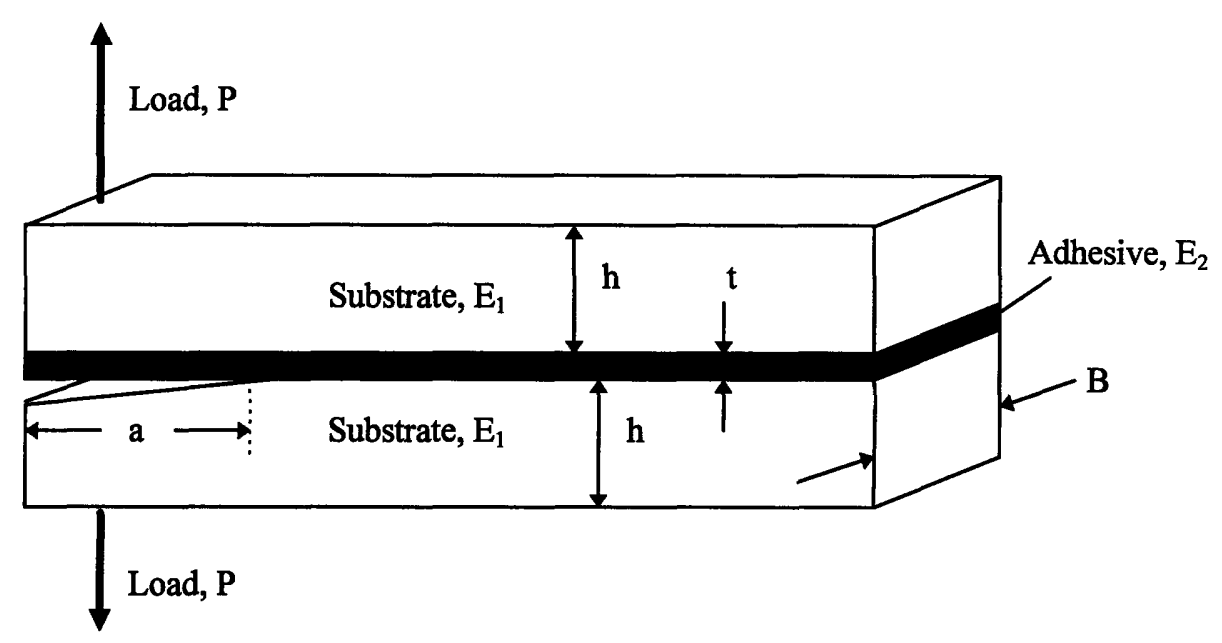

Figure 2.6 - Schematic drawing of the DCB geometry

The previous solutions for strain energy release rate ignore the presence of the adhesive layer and use the solution for a homogeneous specimen. This approach introduces minimal error for adhesive layers that are thin relative to the substrate height (i.e. $t / h<0.08$ ) [33], but becomes invalid as the thickness of the adhesive layer is increased. Recently, an exact analytical solution for the interfacial strain energy release rate of a tri-layer DCB specimen has become available [34]. The solution is based upon the following assumptions: linear elasticity (i.e. small deformations), a fully developed crack across the specimen width, plane stress conditions, only one interface failing during crack propagation (i.e. no crack-jumping between interfaces), and negligible strain energy associated with transverse shear. Strain energy release rate is calculated by determining the potential energy function (stored strain energy minus work of displacement) and differentiating with respect to crack area. For the tri-layer DCB specimen (Figure 2.6), the interfacial strain energy release rate is given by:

$$
G=\frac{6 P^{2} a^{2}}{E_{1} B^{2} h^{3}}+\frac{E_{1} P^{2} a^{2}}{6 D^{2}} \cdot\left(Y_{3}^{3}-Y_{2}^{3}\right)+\frac{E_{2} P^{2} a^{2}}{6 D^{2}} \cdot\left(Y_{1}^{3}-Y_{2}^{3}\right)
$$


where $Y_{1}=-\left(\frac{h+t}{2}\right)$,

$$
\begin{aligned}
& Y_{2}=Y_{1}+t, \\
& Y_{3}=\frac{h+t}{2}, \text { and } \\
& D=B \cdot\left[\frac{E_{2}}{3} \cdot\left(Y_{2}^{3}-Y_{1}^{3}\right)+\frac{E_{1}}{3} \cdot\left(Y_{3}^{3}-Y_{2}^{3}\right)\right] .
\end{aligned}
$$

Note that $Y_{1}, Y_{2}, Y_{3}$, and $D$ are all constants that depend upon specimen geometry and material properties only. Equation 2.24 is an exact solution for the interfacial strain energy release rate, and is valid for any adhesive layer thickness.

\subsection{Fatigue Crack Propagation}

The fracture mechanics concepts discussed so far are useful for describing the conditions of unstable crack growth under static loads. However, most engineering components experience cyclic, as well as static, loading conditions. Also, under oscillating loads, structures will fail at stress levels much lower than they can withstand under static loading [1]. This process is known as fatigue. The primary mechanism for such failure is the initiation and subsequent propagation of a crack. Therefore fracture mechanics is well suited for the study of fatigue.

\subsubsection{Analysis of Fatigue Crack Propagation Data}

Although the stress intensity factor was originally developed to define the onset of unstable crack growth, Paris and his co-workers [35] proposed that it can also be used to characterize fatigue crack propagation (FCP). Their approach related the fatigue crack growth rate to the stress intensity range, $\Delta K=K_{\max }-K_{\min }$, with a general expression of the form:

$$
\frac{d a}{d N}=f(\Delta K)=f(Y \Delta \sigma \sqrt{a})
$$

where a is the crack length, $N$ is the number of fatigue cycles, $\sigma$ is the far-field stress, and $Y$ is the geometric factor. 
Shortly after introducing fracture mechanics into the field of fatigue analysis, it was recognized [36] that the majority of the plotted growth rate vs. stress intensity range data agrees with an equation of the form:

$$
\frac{d a}{d N}=A(\Delta K)^{m}
$$

in which $A$ and $m$ are a function of material and test conditions. Equation 2.26 has come to be known as the Paris Equation. The Paris equation can also be re-expressed in terms of the strain energy release rate range, $\Delta G=G_{\max }-G_{\min }$, by

$$
\frac{d a}{d N}=B(\Delta G)^{n}
$$

where $B$ and $n$ are also parameters which depend upon material and test conditions. Even though this approach lacks a mechanistic basis, it has been successfully applied to both metallic and non-metallic materials. However, if the stress intensity, or strain energy release rate, range is sufficiently varied, the data tend to deviate from the equation asymptotically at both high and low $\Delta K$, or $\Delta G$, levels, resulting in a sigmoidal shape as shown in Figure 2.7. For the sake of clarity, the remainder of the discussion on FCP will only consider equation 2.27 and the energy balance approach, since it is more relevant to the current work. However, it should be noted that the concepts apply equally to the stress intensity factor approach.

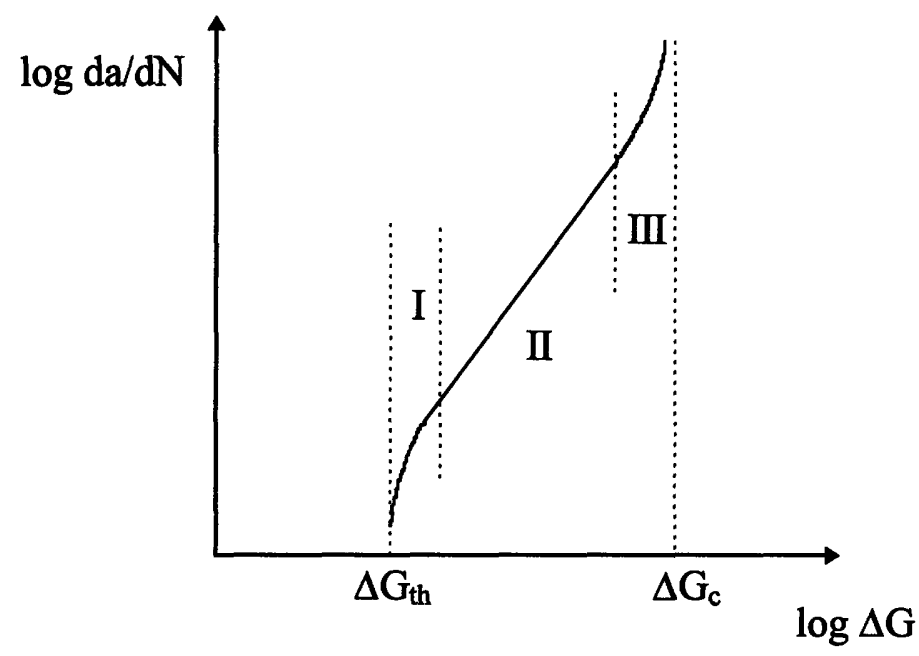

Figure 2.7 - A schematic drawing of a typical, logarithmic scaled da/dN vs. $\Delta \mathrm{G}$ plot with three distinct regimes 
Although most of the FCP data fall within the second regime, as shown in Figure 2.7, the other two regimes are equally important. At high $\Delta G$ levels (regime III), the crack begins to propagate very rapidly as $G_{\max } \rightarrow G_{c}$. At low $\Delta G$ levels (regime I), the crack growth rate deviates from the Paris law again, while approaching a threshold value, $\Delta G_{t h}$, below which the crack does not propagate. $\Delta G_{t h}$ is an important, service-life determining parameter. While $G_{c}$ describes the conditions under which static fracture will occur, $\Delta G_{t h}$ represents the limit below which no fatigue crack propagation will occur. Since FCP can ultimately lead to failure at conditions well below $G_{c}$, the threshold strain energy release rate represents a much more conservative design parameter. In the case of crack propagation at an interface, $\Delta G_{t h}$ should also be very closely related to the intrinsic adhesion forces acting across the interface since at threshold load levels, since plastic dissipative processes are minimal.

\subsubsection{Crack-Tip Shielding Mechanisms}

Oftentimes, the fatigue crack driving force at the crack tip may be lower than the applied, or "far-field," crack driving force $\Delta G$. When this occurs, the crack tip is said to be "shielded" from the applied driving force, which results in a decrease in FCP rate. Therefore, extrinsic mechanisms which promote crack-tip shielding processes can greatly enhance the FCP resistance of a material or material system.

Under monotonic loading, shielding simply results in a reduction of the crack-tip stress intensity. However, under cyclic loading, the effects become more complex and depend upon the crack growth regime [37]. Figure 2.8 illustrates some crack-tip shielding mechanism relevant to the study of fatigue at interfaces. A more complete description of shielding mechanisms may be found elsewhere [38].

Crack deflection (Figure 2.8 a) occurs when obstacles in the way of the crack tip cause the crack to deflect from the nominally mode-I plane. Once deflected, the crack tip no longer experiences the full mode-I component of the far-field load. Therefore, a decrease in FCP rate is observed in all three growth regimes [37]. In the case of crack 
growth along an interface, crack deflection can occur as the result of the interfacial roughness.
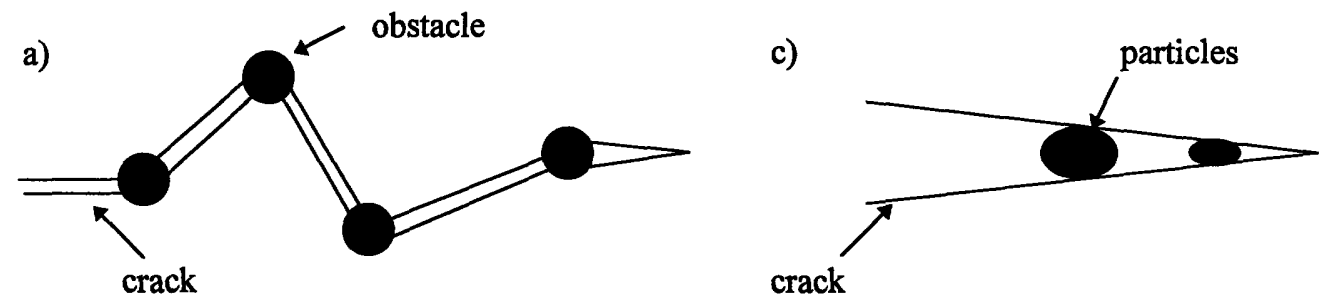

b)

d)
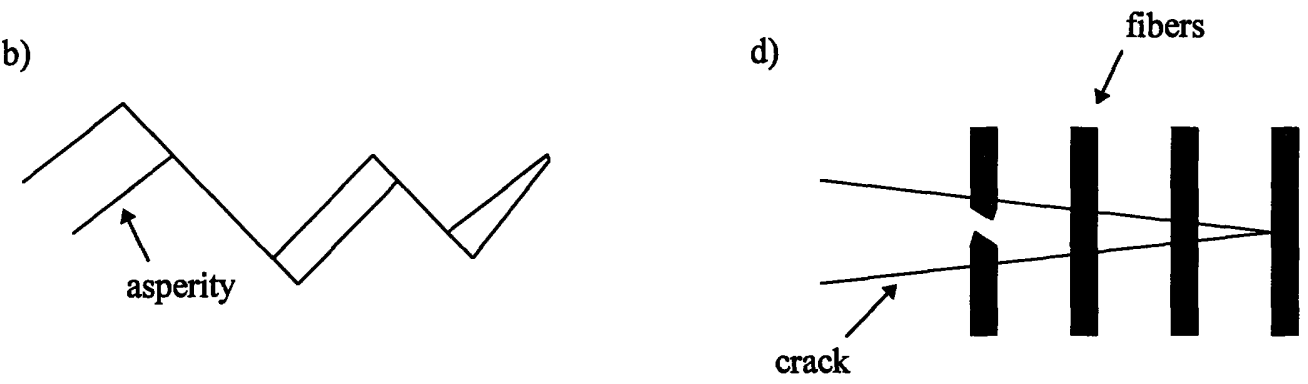

Figure 2.8 - Illustration of crack tip shielding mechanisms: a) crack deflection;

b) roughness induced crack closure; c) crack-bridging by particles; and

d) crack-bridging by fibers

The crack tip can also be shielded if the faces of the crack come into contact at some point during the fatigue cycle. Crack-tip closure can occur via several mechanisms [38], two of which are illustrated in Figure 2.8. Roughness-induced closure (Figure 2.8 b) can occur when asperities on the fracture surfaces contact or slide against each other. Particle-induced closure (Figure $2.8 \mathrm{c}$ ) can occur when foreign particles become wedged in the crack, causing the crack to close. These contact shielding mechanisms are much more effective at lower levels of driving force where the crack tip opening displacements are small. Therefore, the effects of contact shielding are dominant in the threshold regime, but not at higher growth rates.

Another FCP toughening mechanism observed in fiber reinforced polymer composites is fiber bridging (Figure $2.8 \mathrm{~d}$ ). In this mechanism, unbroken fibers span the crack wake, and inhibit crack opening. The energy required to strain and fracture the 
fibers also contributes to the overall toughening of the material system. However, unlike contact shielding and crack deflection, the effects of fiber bridging can be seen at all crack growth regimes, depending on the strength of the fiber/matrix interface [39]. Thus, the analysis of shielding mechanisms becomes more complex in multi-component material systems, especially when crack propagation occurs along an interface.

\subsection{Interfacial Fatigue Crack Propagation}

Although a large body of literature exists for interfacial fracture, very few researchers have investigated fatigue crack propagation at interfaces, with the exception of some very recent work that is discussed in section 2.4.2. However, some investigations of FCP in adhesive joints have been reported within the last 20 years. Most of this work did not deal with adhesive, or interfacial, failure; rather, it focused on cohesive crack propagation with the bulk of the adhesive layer. Nevertheless, this work provided a foundation for the current research and will be discussed next.

\subsubsection{Fatigue Crack Propagation in Adhesive Joints}

Increasing use of adhesives in the aerospace and automotive industries has spurred the development of methods for characterizing the performance of adhesives in bonded structures. Since most engineering components are subjected to cyclic loads, knowledge of the fatigue behavior of bonded joints is essential. Early work by Mostovoy and Ripling [40] established the validity of using a fracture mechanics approach for describing FCP in specimens made from epoxy-bonded aluminum substrates.

Initial investigations of epoxy/aluminum systems by Mostovoy and Ripling [40] and, later, Jablonski [41] employed the tapered double cantilever beam (TDCB) geometry, which is nominally mode I. The height of TDCB substrates is contoured such that $\partial C / \partial a$ is constant with crack extension. Therefore, the strain energy release rate is independent of crack length, and depends only on the substrate width and applied load (see eq. 2.20). Although this geometry aids in the calculation of $G$, the substrates require very precise machining. Their results showed that the power-law relationship between crack growth rate and $\Delta G$ holds [40,41], and that typical FCP behavior such as crack 
closure [41] and a fatigue crack growth threshold [40] were present. Since these adhesive systems exhibited very strong interfaces, all of the fatigue failures were observed to be cohesive in nature.

Mall and coworkers $[42,43,44]$ examined the mode I and mixed-mode FCP behavior of adhesively bonded carbon fiber composite substrates. Mode I experiments were conducted using the DCB geometry; whereas mixed-mode experiments utilized the cracked-lap shear (CLS) geometry [42]. Their results indicated that the total strain energy release rate, $\Delta G_{T}$, described the FCP behavior via a power law relationship as in eq. 2.26. A similar conclusion was reached by Lin and Liechti [45] who used four different geometries to study mixed-mode FCP in bonded aluminum systems. Both groups used ductile adhesives and reported a mix of adhesive and cohesive failure, often on the same specimen. However, neither of these groups reported the presence of a fatigue crack growth threshold.

Later work by Mall and Tan [46] found that the failure locus (adhesive vs. cohesive) was influenced by the ductility of the adhesive. Unlike the previous work on ductile adhesives where failure was mainly cohesive with a small amount of adhesive failure, brittle adhesives tended to fail either completely cohesively or completely adhesively, with no consistent pattern. A considerable reduction in the FCP resistance was observed for the samples that failed adhesively, indicating that the adhesive/metal interface was weaker than the bulk adhesive. Also, the total strain energy release rate could not be used to correlate the FCP data between DCB (mode I) and CLS (mixedmode) geometries indicating a change in mechanism with the brittle adhesives when failure occurred either at the interface or under mixed mode conditions. Again, no threshold data were reported.

Recent research has focused upon parametric studies of the properties of the adhesive joint, FCP testing conditions, and lifetime predictions; however no threshold behavior was investigated. Schmusser [47] used the CLS geometry to investigate the effect of electro-priming and bondline thickness on adhesively bonded steel joints. He also found that $G_{T}$ described the FCP data adequately. In contrast to work done with aluminum adherends, the steel joints were found to fail adhesively at the adhesive/steel 
interface for unprimed specimens and at the primer/steel interface for primed specimens. Bondline thickness was found to have a weak effect on FCP resistance, but priming increased the FCP resistance by 44-242\%. Joseph and co-workers [48] implemented the DCB geometry to study the effects of surface pre-treatment, water soak, fatigue cycle frequency, adhesive thickness, and type of adhesive. Most failures were found to be either cohesive or mixed adhesive/cohesive. Surface treatments (mercaptoester coupling agents) were found to increase the FCP resistance, even after water immersion, and generally resulted in complete cohesive failure. Fatigue frequency did not have a significant effect, but increasing bondline thickness led to improved fatigue resistance. In a recent study, Kinloch and Oseymi [49] used FCP data collected using the DCB geometry to successfully predict the lifetime of single-lap adhesive joints. This was the first time experimental fatigue data was used to model the behavior of a real adhesive joint.

\subsubsection{Fatigue Crack Propagation at Interfaces}

Although some of the work on FCP in adhesive joints reported interfacial (adhesive) failure, none intentionally and systematically examined fatigue crack propagation at the interface. Only very recently have such investigations been reported in the literature.

The first such study investigated nominally Mode I FCP at copper/glass and aluminum/alumina interfaces [50]. For both material systems, crack growth rate exhibited a power-law type dependence on $\Delta G$. Failure in the copper/glass system was found to be truly interfacial, in that no copper could be detected on the glass fracture surface and vice versa. However, in the aluminum/alumina system crack growth did not occur at the interface, but in either the ceramic or the metal, within a few microns of the interface. For both systems, crack growth occurred under cyclic loading at levels well below those required for fast fracture or static fatigue (stress-corrosion). By implanting voids in the copper/glass interface, the authors were able to demonstrate a crack-tip shielding effect induced by crack bridging of bulged metal film segments across the crack. Specimens with implanted voids exhibited crack growth rates many orders of magnitude below those of void-free specimens. Therefore, the crack-tip 
shielding/bridging effect was shown to be a potent toughening mechanism for interfacial fatigue crack growth.

Ritter and co-workers [51] have examined static and fatigue crack propagation at polymer/glass interfaces. Using a four-point flexure geometry, they established that crack propagation could be related to strain energy release rate via a power-law function. Although there was considerable scatter in the data, threshold crack propagation values were reached. FCP at the polymer/glass interface occurred at energy release rates that were two orders of magnitude lower than those required for bulk FCP in epoxy-based adhesives, indicating a lack of energy dissipating processes during interfacial FCP. Although successful, this method relies on monitoring the crack length optically through the glass substrate. Thus, its usefulness only extends to systems having transparent substrates. The same technique was applied to epoxy/PMMA interfaces [52]. However, due to considerable scatter in the data, crack growth rate could not be correlated to $\Delta G$, and no evidence of a fatigue mechanism could be detected.

An experimental technique has also been developed for studying mixed-mode crack growth at solder/copper interfaces [53]. Using the flexural peel geometry, mixed mode loadings of $30^{\circ}<\Psi<60^{\circ}$ were obtained. Investigations into the effect of modemix [54] and interface roughness [55] revealed that crack face sliding was a significant mechanism during interfacial FCP. Interface roughness was found to play an important role in determining the crack growth threshold $\left(\Delta G_{t h}\right)$ where crack face sliding was dominant, but had less of an impact at higher growth rates where larger crack openings prevented crack face sliding. Increasing the phase angle of the load-mix was found to increase both the $\Delta G_{t h}$ and the $\mathrm{FCP}$ resistance at higher growth rates.

\subsubsection{Purpose of this Investigation}

Thus, a limited body of knowledge now exists for FCP at polymer/ceramic, polymer/polymer, metal/metal, and metal/ceramic interfaces. However, polymer/metal interfaces remain, for the most part, uninvestigated. As mentioned previously, the integrity of polymer/metal interfaces under cycling loading is of critical importance in microelectronic packaging. Interfacial fatigue crack propagation seems to be a viable 
method for assessing interfacial adhesion, but no one has yet applied it to microelectronic packaging materials. Therefore, it is the intent of this work to develop a method for studying fatigue crack propagation at polymer/metal interfaces, gain an understanding of the mechanisms of interfacial crack propagation in such systems, and determine the implications for microelectronic packaging performance. 


\section{Chapter 3}

\section{Experimental}

\subsection{Material Systems}

The specimens used in this study consisted of metal substrates in the form of cantilever beams bonded together with a thin layer of polymer encapsulant. Since the goal of this work was to simulate the actual heat slug/encapsulant interface found in the microelectronic package, test specimens were designed and processed as closely to package specifications as possible.

\subsubsection{Substrates}

Copper beam test specimens were acquired from the same vendor who manufactures the package heat slugs. The substrate base metal was OFHC copper $\left(E=120 \mathrm{GPa}, \sigma_{y}=260\right.$ $\mathrm{MPa}, \alpha=16.7 \cdot 10^{-6} /{ }^{\circ} \mathrm{C}$ ). The bonding surfaces were electroplated to a nominal plating thickness of approximately three micrometers. Two metal plating processes were investigated: dull, clear nickel and luster copper. To investigate the effect of surface roughness, one lot of each type of plated specimens was subjected to a "blast" treatment after plating to induce a macroscopically rough surface. Specimen beam dimensions, given in Figure 3.1, were chosen such that yielding did not occur in the copper during double cantilever beam (DCB) testing. The DCB specimens were also made excessively thick to reduce beam deflection to negligible levels. 


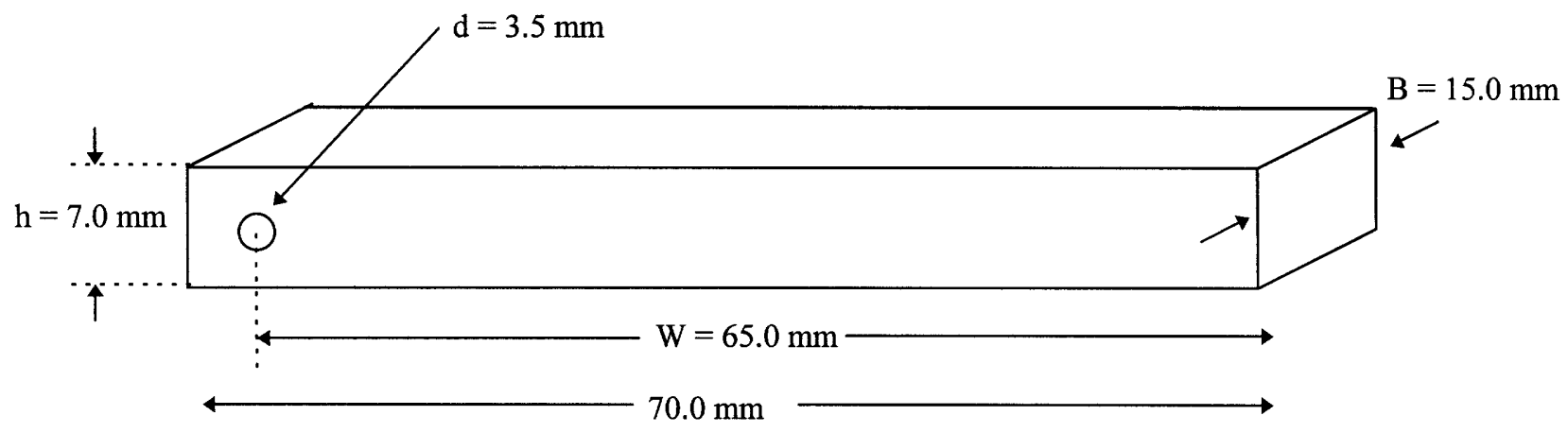

Figure 3.1 - Illustration of the double cantilever beam (DCB) substrate geometry, indicating critical dimensions

\subsubsection{Polymer Encapsulants}

Three candidate encapsulants were used in the screening portion of this study. The materials were procured through Intel Corp., Chandler, AZ, from confidential vendors, and will be referred to as materials A, B, and C. Encapsulants B and C were both single component liquid systems. Whereas, material A came in a solid pellet which was melted into a liquid prior to curing. All three were highly filled materials, containing $70-80 \mathrm{wt} \%$ silica particles. Table 3.1 summarizes the basic chemistry, filler loading, elastic modulus and recommended cure schedule for each encapsulant.

Table 3.1 - Encapsulant chemistry, filler loading, elastic modulus, and recommended cure schedules

\begin{tabular}{|c|c|c|c|c|}
\hline Encapsulant & $\begin{array}{c}\text { Basic } \\
\text { chemistry }\end{array}$ & $\begin{array}{c}\text { Filler loading } \\
\text { (silica particles) }\end{array}$ & $\begin{array}{c}\text { Elastic } \\
\text { modulus (GPa) }\end{array}$ & $\begin{array}{c}\text { Recommended } \\
\text { cure schedule }\end{array}$ \\
\hline A & $\begin{array}{c}\text { crystalline } \\
\text { epoxy }\end{array}$ & $70-80 \mathrm{wt} \%$ & 8.0 & $\begin{array}{c}125^{\circ} \mathrm{C} \text { for } 15 \\
\text { min., } 150^{\circ} \mathrm{C} \text { for } \\
2 \mathrm{hr} .\end{array}$ \\
\hline B & $\begin{array}{c}\text { phenol novalac } \\
\text { epoxy }\end{array}$ & $70-80 \mathrm{wt} \%$ & 9.5 & $165^{\circ} \mathrm{C}$ for $3 \mathrm{hr}$. \\
\hline C & $\begin{array}{c}\text { anhydride } \\
\text { epoxy }\end{array}$ & $70-80 \mathrm{wt} \%$ & 9.0 & $\begin{array}{c}110^{\circ} \mathrm{C} \text { for } 80 \\
\min ., 165^{\circ} \mathrm{C} \text { for } \\
3 \mathrm{hr} .\end{array}$ \\
\hline
\end{tabular}




\subsection{Substrate / Polymer Surface Characterization}

Several techniques were used to characterize the physical and chemical properties of the various interface materials. Contact angle goniometry was used to determine the surface energies of the metal substrates and encapsulants cured against air. Laser profilometry and SEM were used to quantify the roughness of the substrates.

\subsubsection{Contact Angle Goniometry}

Polymer encapsulants test specimens were drawn on glass slides using a cleaned metal blade. They were then cured in an air-circulating oven according to the recommended cure profiles given in Table 3.1. The metal substrates were tested as-received from the supplier.

A relatively new procedure was used to calculate the thermodynamic work of adhesion for the various polymer/substrate pairs. This technique, put forward by R.J. Good and coworkers $[13,14,15]$, utilizes the "three liquid procedure" to measure the surface energy of the interface materials, including acid, base, and dispersive components. The surface energy data are then used to calculate the thermodynamic work of adhesion of the substrate/polymer pairs.

The three liquid procedure utilizes three liquids, one apolar and two polar, with known acidity and basicity to probe the surface of interest. Contact angles of the three liquids are measured, and calculations reveal the complementary acid, base, and apolar (dispersive) nature of the surface. Applying equation 2.6 to the contact angles formed by liquids 1 (apolar), 2 (polar), and 3 (polar) yields the following set of simultaneous equations.

$$
\begin{gathered}
4 \gamma_{S}^{d}=\gamma_{L 1}^{d}\left(1+\cos \theta_{1}\right)^{2} \\
W_{S L 2}^{\text {Total }}=\gamma_{L 2}\left(1+\cos \theta_{2}\right)=2 \cdot\left[\left(\gamma_{S}^{d} \gamma_{L 2}^{d}\right)^{\frac{1}{2}}+\left(\gamma_{S}^{+} \gamma_{L 2}^{-}\right)^{\frac{1}{2}}+\left(\gamma_{S}^{-} \gamma_{L 2}^{+}\right)^{\frac{1}{2}}\right] \\
W_{S L 3}^{\text {Total }}=\gamma_{L 3}\left(1+\cos \theta_{3}\right)=2 \cdot\left[\left(\gamma_{S}^{d} \gamma_{L 3}^{d}\right)^{\frac{1}{2}}+\left(\gamma_{S}^{+} \gamma_{L 3}^{-}\right)^{\frac{1}{2}}+\left(\gamma_{S}^{-} \gamma_{L 3}^{+}\right)^{\frac{1}{2}}\right]
\end{gathered}
$$


Since the acid, base, and dispersive surface energy components of the three liquids are known, the corresponding components of the surface material are found by simultaneously solving equations $3.1-3.3$. The calculated surface energy components can then be used to calculate the thermodynamic work of adhesion for substrate-adhesive pairs using equation 2.7 .

The three liquids used in this study were diiodomethane (apolar), deionized water (polar), and glycerol (polar). The surface energy parameters for these liquids may be found in Table 3.2 .

Table 3.2 - Surface energy parameters for the three liquids used in this study [6]

\begin{tabular}{|l|l|l|l|}
\hline Liquid & $\gamma^{\mathbf{d}}, \mathbf{m J} / \mathbf{m}^{2}$ & $\gamma^{+}, \mathbf{m J} / \mathbf{m}^{2}$ & $\gamma^{-}, \mathbf{m J} / \mathbf{m}^{2}$ \\
\hline Diiodomethane & 50.8 & 0.0 & 0.0 \\
\hline Deionized water & 21.8 & 25.5 & 25.5 \\
\hline Glycerol & 34.0 & 3.92 & 57.4 \\
\hline
\end{tabular}

Contact angles were measured using an automated video contact angle goniometer developed by Connelly Applied Research (Nazareth, PA). This instrument advances the liquid with a computer controlled syringe pump, stores video images from a long-range microscope, and determines the contact angle using a proprietary, sub-pixel interpolation method. A given drop was advanced seven times at a pumping rate of $0.5 \mu \mathrm{l} / \mathrm{sec}$. An initial image of the drop was captured at the instant before the advance occurred. The computer then stopped the growth of the drop for $60 \mathrm{sec}$. and obtained a second image of the drop at meta-stability. Only the second image was used in this study. This process continued, and after seven image pairs had been stored, the contact angles were measured for both sides of each drop. The contact angle data were then exported to a spread sheet for calculation of the surface parameters and thermodynamic work of adhesion.

\subsubsection{Laser Profilometry}

The surface roughness of the DCB substrates was measured using a UBM laser profilometer. Two scans, one in the x-direction and one in the y-direction, were acquired at three different locations of each specimen to yield a total of six measurements per 
specimen. Each scan traversed a distance of $2 \mathrm{~mm}$. The data from each scan is stored as a height vs. scanning distance profile and is filtered and corrected for surface tilt prior to data analysis. Several roughness parameters are calculated by the instrument, but only two are used in this study. The first, $R_{q}$, is defined as the root mean square of the individual height measurements, $y_{i}$.

$$
R_{q}=\sqrt{\frac{1}{n} \sum_{i=1}^{n} y_{i}^{2}}
$$

The second parameter, $S$, is defined as the average of the individual distances between local peaks in the profile $\left(D_{i}\right)$.

$$
S=\frac{1}{n} \sum_{i=1}^{n} D_{i}
$$

For the purpose of comparison, a relative roughness parameter $R$ was defined:

$$
R=\frac{R_{q}}{S}
$$

to account for both peak height and peak-to-peak spacing.

\subsubsection{SEM}

Scanning electron microscopy (SEM) was used to provide both plan and cross sectional views of the substrate surface roughness. The cross section samples were cut on a diamond wheel, polished, and sputter coated with a thin layer of gold (15 sec. sputter time). No gold coating was used on the plan view samples. A Cambridge S360 SEM was used to obtain images of varying magnification under the following conditions: an accelerating voltage of $15-20 \mathrm{kV}$, a working distance of $19-28 \mathrm{~mm}$, and no tilt.

\subsection{Specimen Preparation}

The DCB specimens used in this study were fabricated using the following general method. One substrate was sprayed with a dry lubricant mold release agent over the first $24.5 \mathrm{~mm}$ of one end to create a precrack along the substrate-polymer interface. Another substrate was selected, and the two were placed in a specially designed mold, illustrated in Figure 3.2, such that the bonding surfaces were vertical and facing each other. With 
one mold sidebar fixed, the other sidebar was slid into contact with the ends of the substrates and fixed into place using screws threaded into the baseplate. This process aligned the bonding surfaces of the substrates. Next, one substrate was aligned and fixed in place using the top clamp. Two $10 \mathrm{mil}(25.4 \mu \mathrm{m})$ shims were then inserted at either end of the bonding area on the clamped substrate, and the free substrate was brought into contact with the shims. The bottom clamps were used to secure the free substrate, and the shims were removed from the bonding area. The substrates were now aligned and held in place with a bond line thickness of 10 mils.

The mold assembly was then transferred to a hot plate for adhesive dispensing. The mold was heated to a temperature of approximately $80^{\circ} \mathrm{C}$, as monitored by a thermocouple. The encapsulant was dispensed from a syringe along the bond line and flowed into the bond region. The dispensing continued until the bonding region was filled with material. A final fillet of encapsulant was dispensed onto the bond line to feed any shrinkage during cure (see Figure 3.3). Finally, the mold was transferred to an aircirculating oven, and the specimens were cured according to the appropriate cure profile (see Table 3.1).

Once the cure was complete, the specimens were removed from the mold. Any excess encapsulant was removed with sandpaper. The specimens were then sent to an independent contractor (Ronald Hall, San Francisco, CA) for attachment of the KrakGage $^{\circledR}$. The Krak-Gage ${ }^{\circledR}$ was mounted such that the mouth of the gage was coincident with the end of the precrack region. 


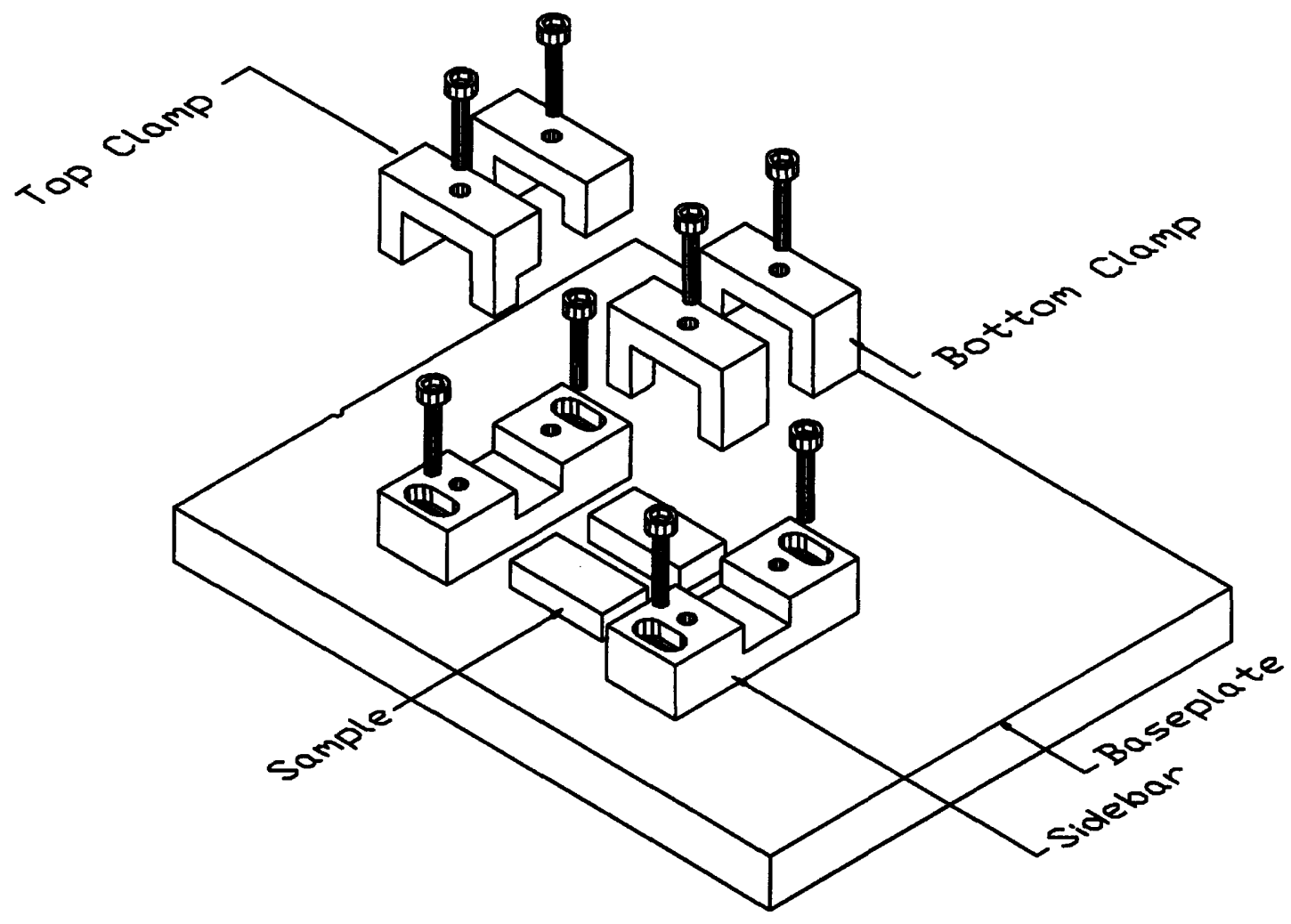

Figure 3.2 - Illustration of the mold used for fabricating DCB specimens (Note: not drawn to scale.)

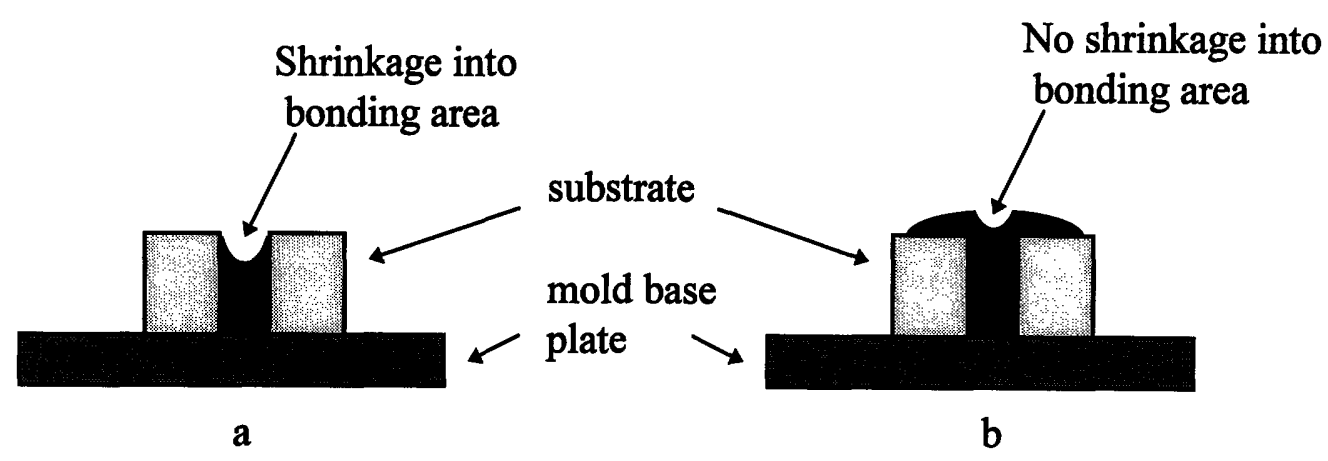

Figure 3.3 - Illustration of typical cure shrinkage, a) without a fillet and (b) with a fillet (note: not drawn to scale) 


\subsection{Fatigue Crack Propagation Experiments}

\subsubsection{Precracking}

The dry lubricant mold release agent was found to create an area of weak adhesion, not a true precrack. Therefore, fatigue precracking was necessary to introduce a sharp, interfacial crack. To facilitate precracking the specimen was notched using a diamondimpregnated wire saw ( 8 mil diameter wire). All encapsulant ahead of the loading axis was removed. Then, two cuts were made from either side of the bond line to produce a Chevron "v-notch" type cross-sectional profile (see Figure 3.4). The cut was made such that a crack of approximately $0.2 \mathrm{~mm}$ was registered in the Krak-Gage ${ }^{\circledR}$. Aluminum loading blocks were then bonded to the top and bottom of the substrate with a fast-curing cyanoacrylate adhesive.

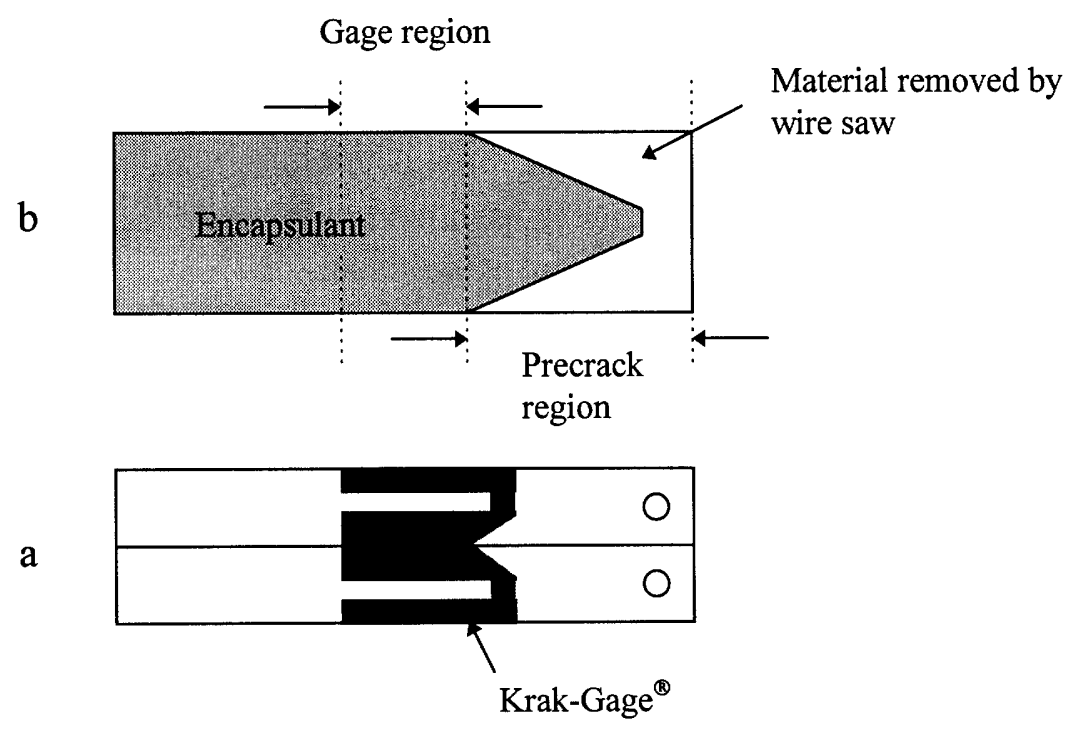

Figure 3.4 - a) Side view of DCB specimen showing Krak-Gage ${ }^{\circledR}$ location b) Top-down "cut-away" view of DCB specimen showing the precrack and gage regions

Fatigue Crack Propagation (FCP) tests were conducted on an MTS 858.02 servohydraulic test system equipped with an MTS 458 controller. A Krak-Gage ${ }^{\circledR} /$ FRACTOMAT $^{\circledR}$ system (Hartrun Corporation, St. Augustine, FL) was used to monitor crack length. The fatigue testing was automated through the use of a personal computer running CrackWatch ${ }^{\circledR}$ software developed by Interactive Software Controls, Stanford, 
CA. All tests were run under load control in the $245 \mathrm{~N}$ range of a $2450 \mathrm{~N}$ load cell. The load signal was monitored via a digital oscilloscope.

Fatigue precracking was performed in constant load amplitude $(\Delta P)$ mode under the following conditions: sinusoidal waveform, frequency of $10 \mathrm{~Hz}$, and ratio of minimum load to maximum load $(R)$ of 0.1 . $\Delta P$ was gradually increased every few hours until $0.1 \mathrm{~mm}$ of crack growth was registered in the gage. In general, the final $\triangle P$ of the precracking procedure was on the order of $50-75 \%$ of the $\Delta P$ required to fracture the specimen.

Once a crack was detected in the gage, a constant $\Delta K$ control mode was selected on the software. The control software calculated $K$, assuming a homogenous specimen, i.e. neglecting the adhesive layer in the calculation of $K$. The non-dimensional relationship between $K$ and load $(P)$ for the DCB geometry is given by [56]

$$
\frac{K h^{1 / 2}}{P}=\sqrt{12}\left(\frac{a}{h}+0.673\right)+\sqrt{\frac{2 h}{\pi a}}-\left(0.815\left(\frac{a}{h}\right)^{0.619}+0.429\right)^{-1}
$$

where $a=$ crack length, and

$h=$ half height of the DCB specimen (see also Figure 3.1).

Equation 3.11 applies for all values of $a / h$, with $c / h>2$, where $c$ is the length of the uncracked ligament (i.e. $W-a$ ). The specimen was fatigued at a constant $\Delta K$ until an additional $0.5 \mathrm{~mm}$ of crack growth was achieved, to ensure that the crack tip was well within the gage section, and that the crack growth was stable. $\Delta K$ was chosen such that the load levels were equal to or greater than the loads experienced during precracking in order to avoid load interactions.

\subsubsection{Fatigue Crack Growth Testing}

The test was then placed in computer-controlled decreasing $\Delta \mathrm{K}$ mode. The frequency and $\mathrm{R}$ ratio remained unchanged at $10 \mathrm{~Hz}$ and 0.1 . The software gradually reduced the load according to a load-shedding scheme of

$$
\Delta K=\Delta K_{0} \exp \left[C^{*}\left(a-a_{0}\right)\right]
$$

where $\Delta K=$ instantaneous value of stress intensity factor range $\left(K_{\max }-K_{\min }\right)$, 
$a=$ instantaneous value of crack length,

$\Delta K_{0}=$ initial value of stress intensity range,

$a_{0}=$ initial value of crack length, and

$C^{*}=$ normalized stress intensity gradient $((d K / d a) / K)$,

by setting $C^{*}$ to -0.1 per mm of crack extension. The crack growth rate ( $d a / d N$, where $N$ is cycles) was calculated by the software according to an incremental polynomial procedure. In this procedure, an interval of crack extension is defined. The software then fits a quadratic polynomial to the $a$ vs. $N$ data over the interval, and calculates the growth rate from the derivative at the mid-point of the interval. The interval length was set to small values (e.g. $0.1 \mathrm{~mm}$ ) at slow growth rates (e.g. $10^{-6} \mathrm{~mm} /$ cycle) and large values (e.g. $0.2 \mathrm{~mm}$ ) at high growth rates (e.g. $10^{-4} \mathrm{~mm} /$ cycle) to provide a consistent number of data points over the range of tested $\Delta K$.

After the crack growth rate decreased to $\sim 10^{-7} \mathrm{~mm} /$ cycle, the test was continued under increasing $\Delta K$ conditions by setting $C^{*}$ to +0.1 per mm of crack extension. The initial value of $\Delta K$ for the increasing portion was set equal to the starting $\Delta K$ from the decreasing portion of the test. Data was collected until a crack growth rate of $10^{-3}$ $\mathrm{mm} / \mathrm{cycle}$ was reached, or until the crack had propagated through the gage region.

\subsubsection{Data Analysis}

The load vs. crack length data recorded by the software were used to calculate the strain energy release rate $(G)$. The maximum $\left(P_{\max }\right)$ and minimum $\left(P_{\min }\right)$ loads were calculated from the recorded load amplitude $(\triangle P)$ using

$$
P_{\max }=\frac{\Delta P}{1-R}
$$

and

$$
P_{\min }=\frac{R(\Delta P)}{1-R} .
$$

The applied strain energy release rate range $\left(\Delta G=G_{\max }-G_{\min }\right)$ was then calculated in accordance with ASTM D3433 [57] by 


$$
\Delta G=\frac{4\left(P_{\max }^{2}-P_{\min }^{2}\right)\left(3 a^{2}+h^{2}\right)}{E_{1} B^{2} h^{3}}
$$

where $a=$ crack length,

$h=$ DCB specimen half-height,

$B=\mathrm{DCB}$ specimen thickness, and

$E_{1}=$ Young's modulus of the DCB substrate material.

Note that equation 3.11 represents the homogenous strain energy release rate which is valid only for thin adhesive layers. Strain energy release rates were also calculated from the exact solution given in Section 2.2.4, equation 2.24. The exact interfacial applied strain energy release rate range is given by:

$$
\Delta G=\left(P_{\max }^{2}-P_{\min }^{2}\right)\left[\frac{6 a^{2}}{E_{1} B^{2} h^{3}}+\frac{E_{1} a^{2}}{6 D^{2}} \cdot\left(Y_{3}^{3}-Y_{2}^{3}\right)+\frac{E_{2} a^{2}}{6 D^{2}} \cdot\left(Y_{1}^{3}-Y_{2}^{3}\right)\right]
$$

where $Y_{1}=-\left(\frac{h+t}{2}\right)$,

$$
\begin{aligned}
& Y_{2}=Y_{1}+t, \\
& Y_{3}=\frac{h+t}{2}, \text { and } \\
& D=B \cdot\left[\frac{E_{2}}{3} \cdot\left(Y_{2}^{3}-Y_{1}^{3}\right)+\frac{E_{1}}{3} \cdot\left(Y_{3}^{3}-Y_{2}^{3}\right)\right] .
\end{aligned}
$$

For the material systems investigated here, the difference between the homogenous strain energy release rate and the exact interfacial strain energy release rate was very small (see Appendix). Therefore, either solution could be used, but to be consistent, the data reported in Chapter 4 were calculated using the exact solution. Interfacial crack growth rate data were then plotted vs. the applied strain energy release rate range. 


\section{Chapter 4}

\section{Results and Discussion}

\subsection{Surface Energy / Thermodynamic Work of Adhesion}

The pseudo-equilibrium advancing contact angle data for the metallic substrates and polymeric adhesives for each liquid are shown in Table 4.1. Since all values of surface energy and work of adhesion are derived from the measured contact angles, it is important to examine these data closely.

Table 4.1 - Pseudo-equilibrium advancing contact angles of the three liquids on metallic substrates and adhesives (figures in parenthesis represent the standard deviations)

\begin{tabular}{|llll|}
\hline Substrate/Adhesive & Diiodomethane & D.I. Water & Glycerol \\
\hline Nickel & $51.26( \pm 2.45)$ & $90.87( \pm 3.35)$ & $93.13( \pm 3.54)$ \\
Nickel - blast & $56.39( \pm 4.45)$ & $109.56( \pm 4.23)$ & $91.01( \pm 5.40)$ \\
Copper & $54.40( \pm 1.98)$ & $90.61( \pm 3.81)$ & $86.90( \pm 1.61)$ \\
Copper - blast & $52.56( \pm 3.03)$ & $101.61( \pm 8.38)$ & $102.77( \pm 4.07)$ \\
\hline Material A & $63.93( \pm 2.82)$ & $93.91( \pm 4.29)$ & $94.84( \pm 3.82)$ \\
Material B & $53.87( \pm 2.80)$ & $94.78( \pm 3.40)$ & $99.13( \pm 5.05)$ \\
Material C & $45.29( \pm 2.69)$ & $87.56( \pm 3.22)$ & $86.59( \pm 5.77)$ \\
\hline
\end{tabular}

Judging from the measured contact angles, it did not appear that the technique was sensitive enough to discriminate between the as-plated nickel and copper surfaces. The average contact angles for water were nearly identical, and if plus/minus one standard deviation error ranges were attached to the average contact angles for the other two liquids, the ranges overlapped. Larger differences in the contact angles on the blast- 
treated substrates were observed, but the considerable scatter in the measurements made the significance of the differences questionable.

Another noteworthy observation was that the scatter (as measured by the standard deviation) of the contact angle measurements was noticeably larger for the blast-treated substrates when compared to the as-plated case. Contact angle measurement techniques generally rely on having a flat, smooth surface. Since the blast treatment roughened the substrate surface, that contact angle measurements could have been affected. Wenzel [58] has shown that surface roughness can change the apparent advancing contact angle observed on a rough surface $\left(\theta_{f}\right)$, compared to that observed on a smooth surface $\left(\theta_{s}\right)$. This change in contact angle may be related by:

$$
\cos \theta_{f}=r_{f} \cos \theta_{s}
$$

where $r_{f}$ is the roughness factor. Mechanistically, this change is caused by diffusion of the liquid into the pores and surface irregularities of the solid. If $\theta_{s}$ on a smooth surface is less than $90^{\circ}$, then roughening the surface will result in $\theta_{f}$ being even smaller. This increases the degree of wetting, and results in an apparent increase in surface energy. However, if $\theta_{s}$ is greater than $90^{\circ}$, the rougher surface will exhibit an even larger $\theta_{f}$, which implies less wetting and a lower apparent surface energy. Therefore, even though the surface chemistry has not changed, roughening can affect the measured contact angle and calculated free energy.

For both the $\mathrm{Cu}$ and $\mathrm{Ni}$ substrates, roughening did not have a significant impact on the contact angle of diiodomethane. The contact angle of glycerol on Ni was similarly unaffected. However, for glycerol on $\mathrm{Cu}$ and water on both $\mathrm{Ni}$ and $\mathrm{Cu}$, the average contact angle increased after the blast treatment. This was consistent with Wenzel's theory since the contact angle on the smooth surface was approximately $90^{\circ}$. However, if it were solely a surface roughness effect, then the change in contact angle should have been seen with all three liquids. Therefore, it was possible that the blast treatment modified the surface chemistry as well as the surface roughness. Since there was no way to separate these effects, it was unclear whether this technique was useful for examining rough surfaces. 
Greater differences seen among the average contact angles for the three polymer adhesives, especially with diiodomethane. The polymer surfaces were very smooth, so roughness effects were not present.

The calculated total surface energies and surface energy components are given in Table 4.2. Based on the measured contact angles, the as-plated Ni had the highest overall surface energy, indicating a very active surface. All of the substrates were found to be more basic than acidic in nature. Interestingly, the blast treated $\mathrm{Ni}$ was found to be almost completely apolar. There are two possible explanations for this. First, it could be an artifact of errors made in measuring the contact angle caused by surface roughness. Second, the blast process could have modified the surface chemistry of the $\mathrm{Ni}$, eliminating all acid and base sites - which seemed highly unlikely. Due to the potential for error in measuring the contact angle, the surface energies should be used with caution.

Table 4.2 - Total surface energies with dispersive and polar components $\left(\mathrm{mJ} / \mathrm{m}^{2}\right)$

\begin{tabular}{|l|c|c|c|c|}
\hline Substrate/Adhesive & $\gamma^{\text {fotal }}$ & $\gamma^{d}$ & $\gamma^{+}$ & $\dot{y}$ \\
\hline Nickel & 41.02 & 33.57 & 1.56 & 8.93 \\
Nickel - blast & 30.67 & 30.65 & 0.00 & 0.08 \\
Copper & 34.36 & 31.79 & 0.28 & 5.97 \\
Copper - blast & 40.01 & 32.84 & 2.82 & 4.57 \\
\hline Material A & 30.81 & 26.32 & 0.65 & 7.73 \\
Material B & 41.38 & 32.09 & 2.48 & 8.68 \\
Material C & 42.20 & 36.86 & 0.88 & 8.08 \\
\hline
\end{tabular}

Materials $\mathrm{C}$ and $\mathrm{B}$ exhibited similar overall surface energies, but the surface of material A seemed much less active. All of the adhesives were more basic than acidic in nature, with material B displaying the highest overall acidity. However, the adhesive specimens tested may not represent the adhesives in actual joints. First, the adhesives were cured against air. Second, since the measurements only probed the surface of the specimen, any low-molecular weight additives (i.e. plasticizers, waxes, de-foaming agents, etc.) that diffused to the surface could affect the contact angle measurements. In a previous study of 20 molding compound formulations, lubricating waxes on the surface molding compound were found to completely obscure contact angle measurements [59]. 
Based on the calculated surface energies, the thermodynamic work of adhesion for each substrate/adhesive pair was computed (see Table 4.3). For each substrate, material $\mathrm{C}$ had the highest work of adhesion, followed by material B, then A. For each adhesive, the $\mathrm{Ni}$ and $\mathrm{Cu}$ blast surfaces exhibited the highest values of work of adhesion. Nickel blast had the lowest, and as-plated copper fell in-between. Therefore, one should expect the best adhesion from material $\mathrm{C}$ on as-plated $\mathrm{Ni}$ and the worst adhesion from material $\mathrm{A}$ on blast treated $\mathrm{Ni}$.

Table 4.3 - Work of adhesion $\left(\mathrm{mJ} / \mathrm{m}^{2}\right)$ for adhesives on various substrates

\begin{tabular}{|l|ccc|}
\hline Substrate & Material A & $\begin{array}{c}\text { Adhesive } \\
\text { Material B }\end{array}$ & Material C \\
\hline Nickel & 71.21 & 82.41 & 83.06 \\
Nickel - blast & 57.26 & 63.61 & 67.75 \\
Copper & 64.73 & 74.69 & 76.05 \\
Copper - blast & 71.58 & 81.55 & 83.14 \\
\hline
\end{tabular}

\subsection{Substrate Surface Roughness}

The results of the laser profilometry measurements are given in Table 4.4. The spacing between local peaks was found to be similar, but the root mean square peak height differed greatly among the four substrates. According to the calculated non-dimensional roughness parameter, $R$, the substrates, in order from the smoothest to the roughest were copper, nickel, blast-treated nickel, and blast-treated copper.

Table 4.4 - Substrate surface roughness parameters

\begin{tabular}{|c|c|c|c|}
\hline Substrate & $\boldsymbol{R}_{q}(\boldsymbol{\mu} \mathbf{m})$ & $\boldsymbol{S}(\boldsymbol{\mu} \mathbf{m})$ & $\boldsymbol{R}$ \\
\hline Nickel & 0.670 & 0.030 & 22.3 \\
Nickel - blast & 2.80 & 0.036 & 77.8 \\
Copper & 0.331 & 0.032 & 10.3 \\
Copper - blast & 2.98 & 0.033 & 90.3 \\
\hline
\end{tabular}

SEM micrographs showing the morphology (plan view) of the various substrates used in this study can be seen in Figures 4.1.-4.2. In the as-plated condition, the copper 
surface appears much smoother than the nickel, which is consistent with the profilometry results. The most striking feature of the blast-treated surfaces is the more damaged appearance. The interfacial roughness can be seen quite well in cross-sectional SEM micrographs of actual specimens made with material B (Figures 4.3-4.4). All of the micrographs show the very high silica filler content in the encapsulant. The roughening of the nickel surface resulted in regularly spaced, rounded bumps. However, the blasting process left a highly damaged copper surface, with large asperities. The magnification of Figure 4.4 was almost too high to make out the details of the blasted copper surface. Figure 4.5 shows the entire thickness of the bond at $150 \mathrm{x}$ for both the as-plated and blasted conditions. The difference was clearly visible at this magnification. 


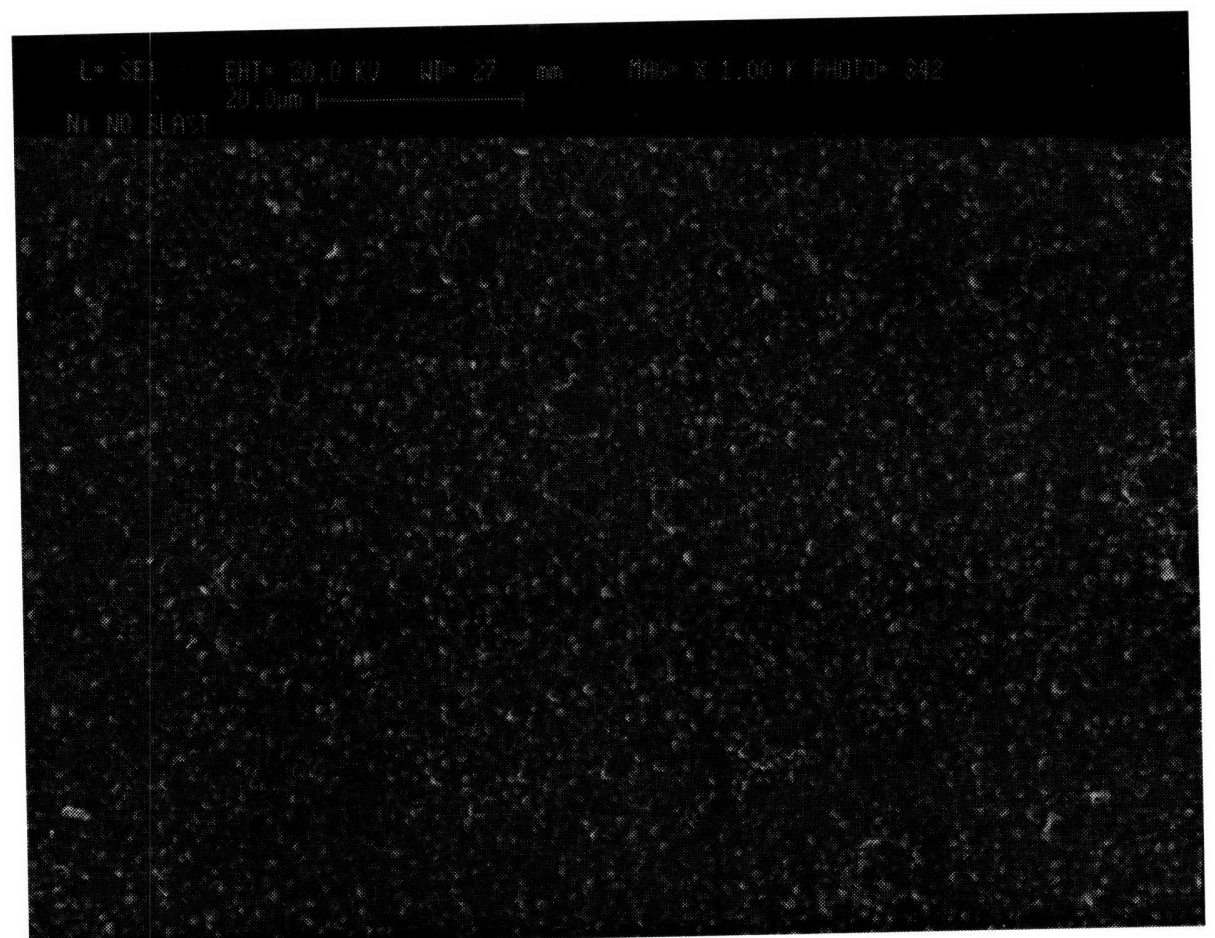

a

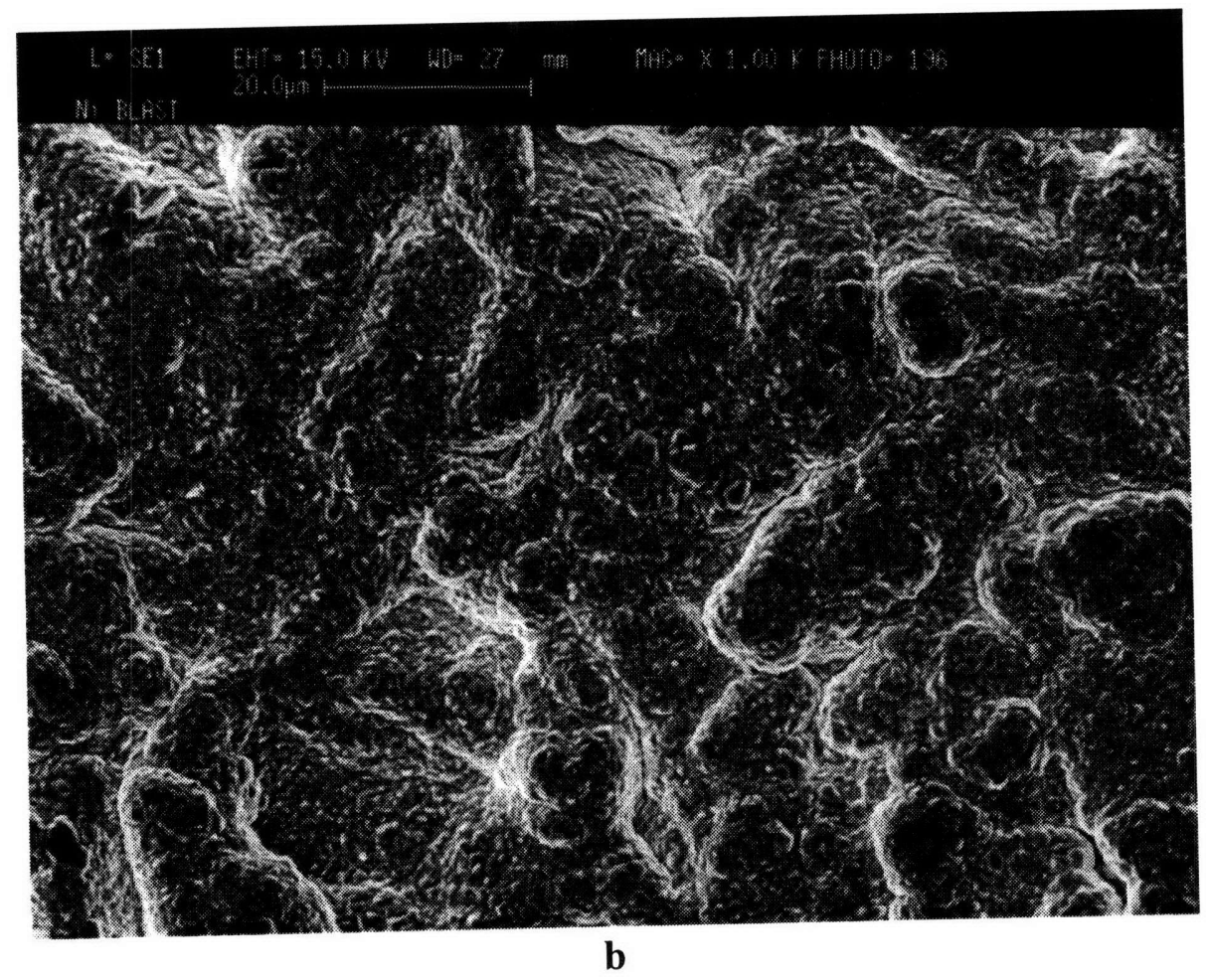

Figure 4.1 - SEM micrographs (1000x) of the nickel plated substrates in the a) as plated and b) blast-treated conditions 


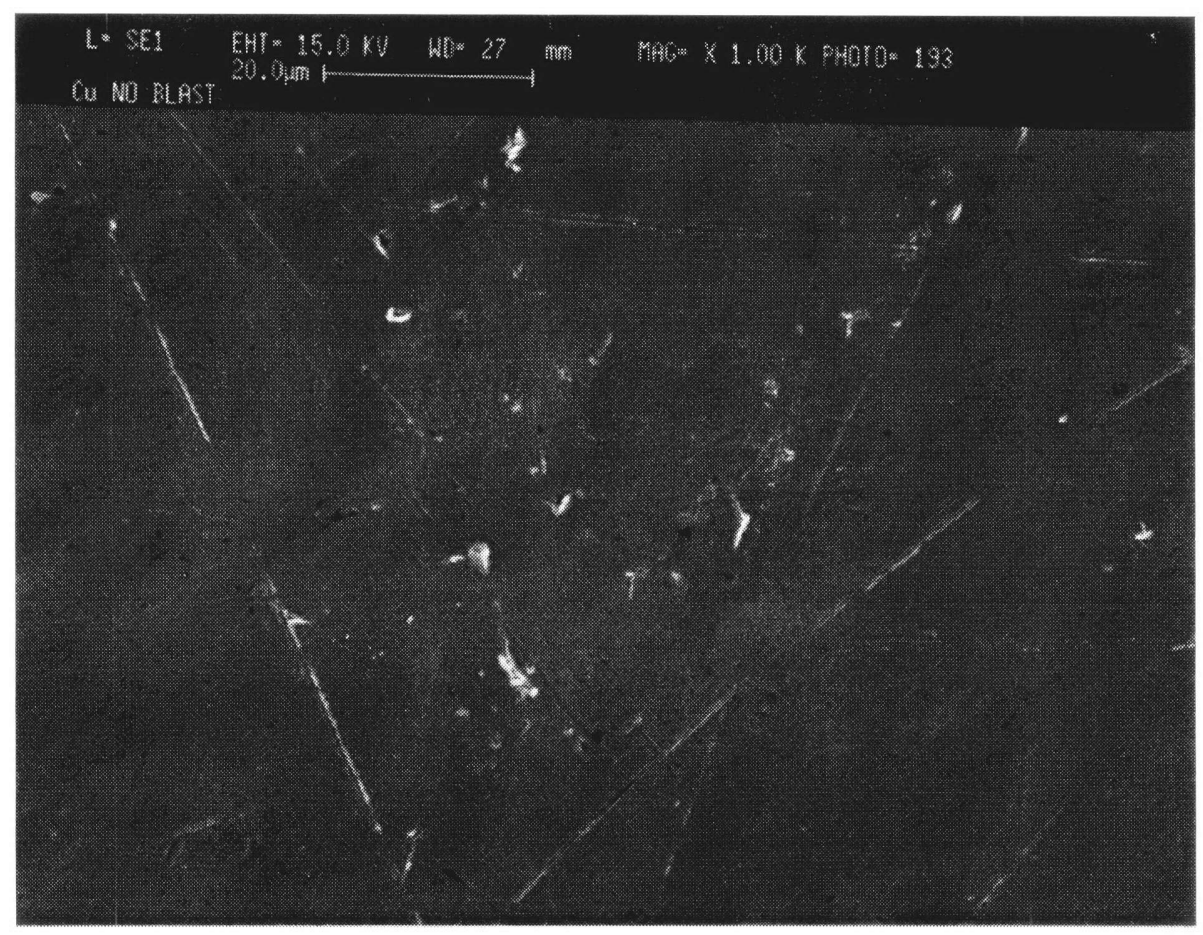

a

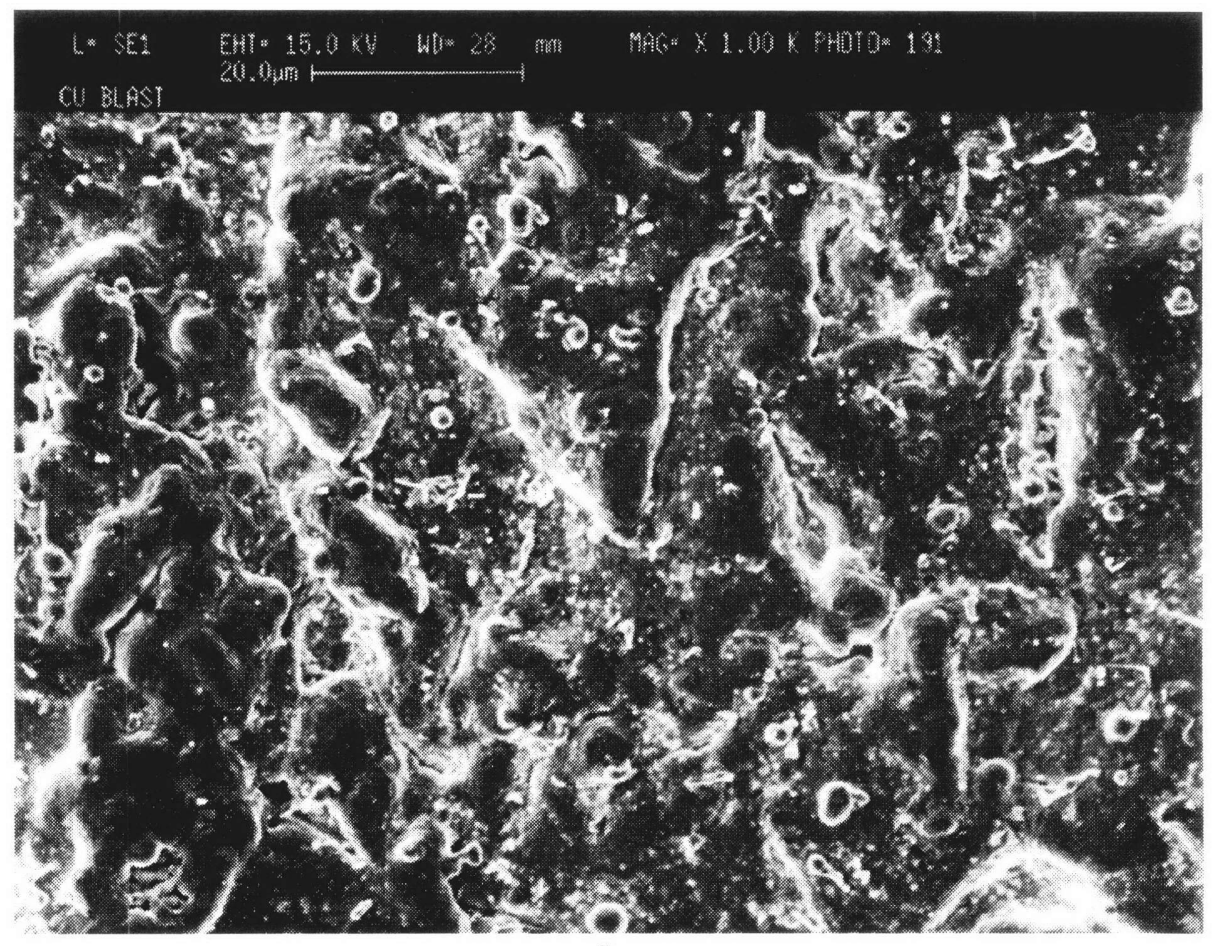

b

Figure 4.2 - SEM micrographs (1000x) of the copper plated substrates in the a) as plated and b) blast-treated conditions 


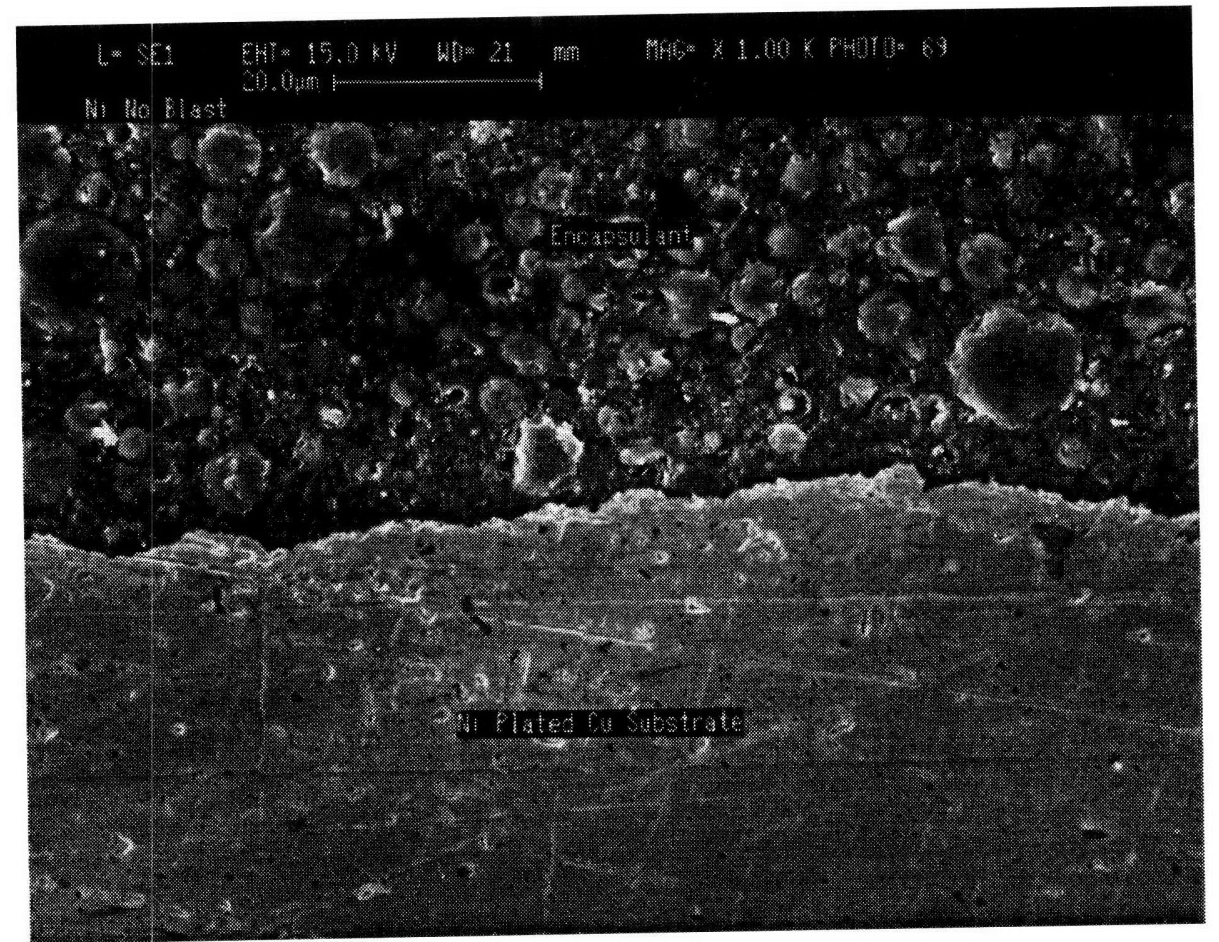

a

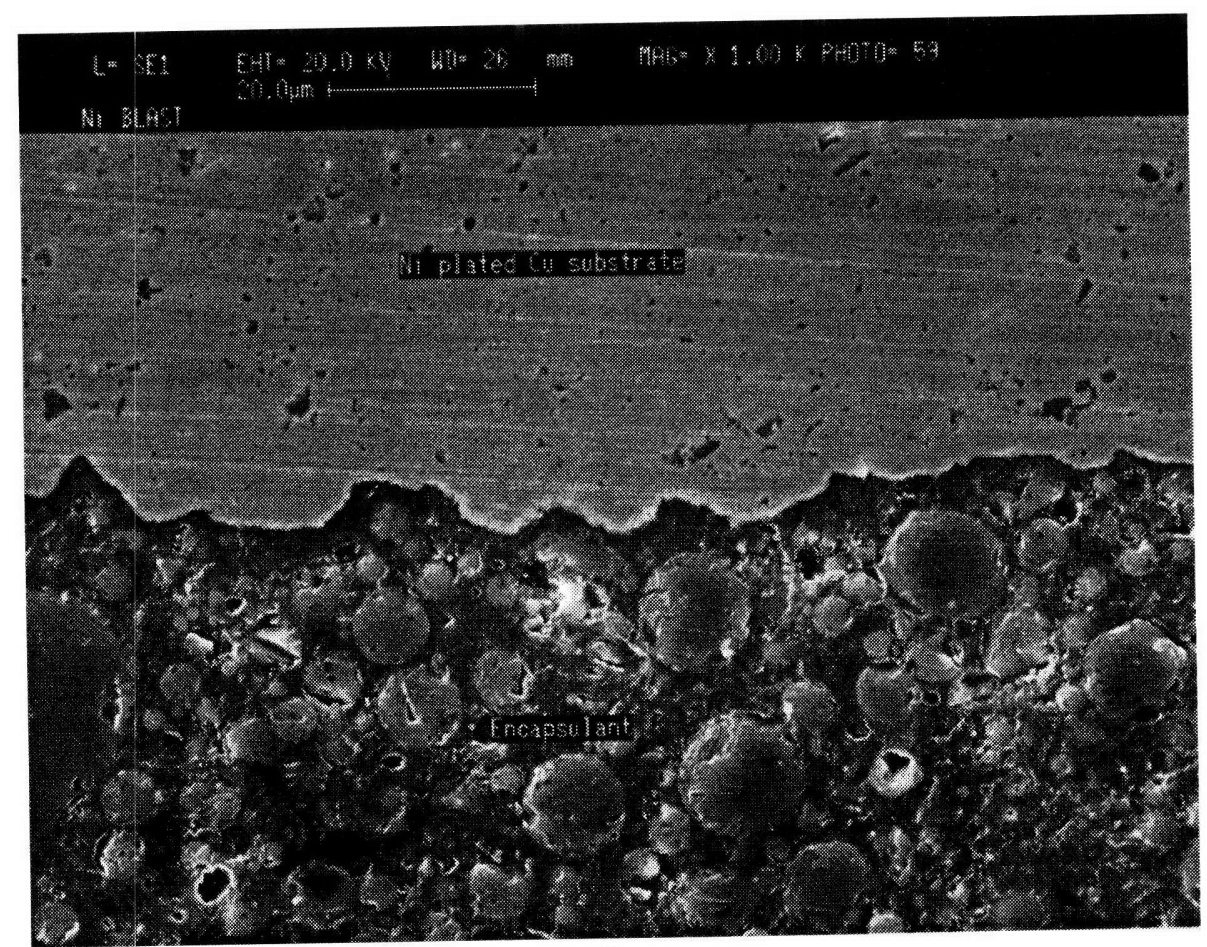

b

Figure 4.3 - SEM micrographs (1000x) of the encapsulant/nickel interface in specimens made from a) as plated and b) blast-treated substrates 


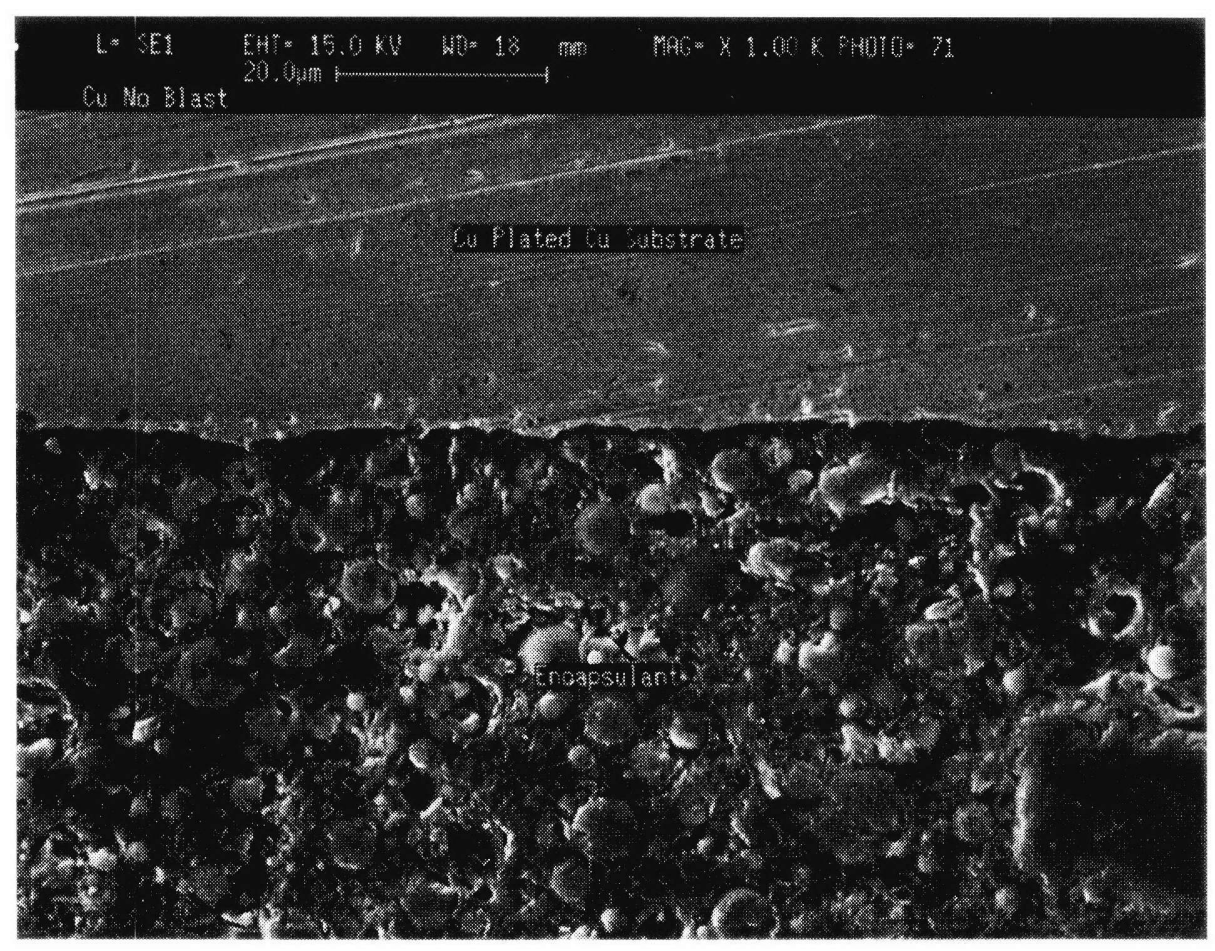

a

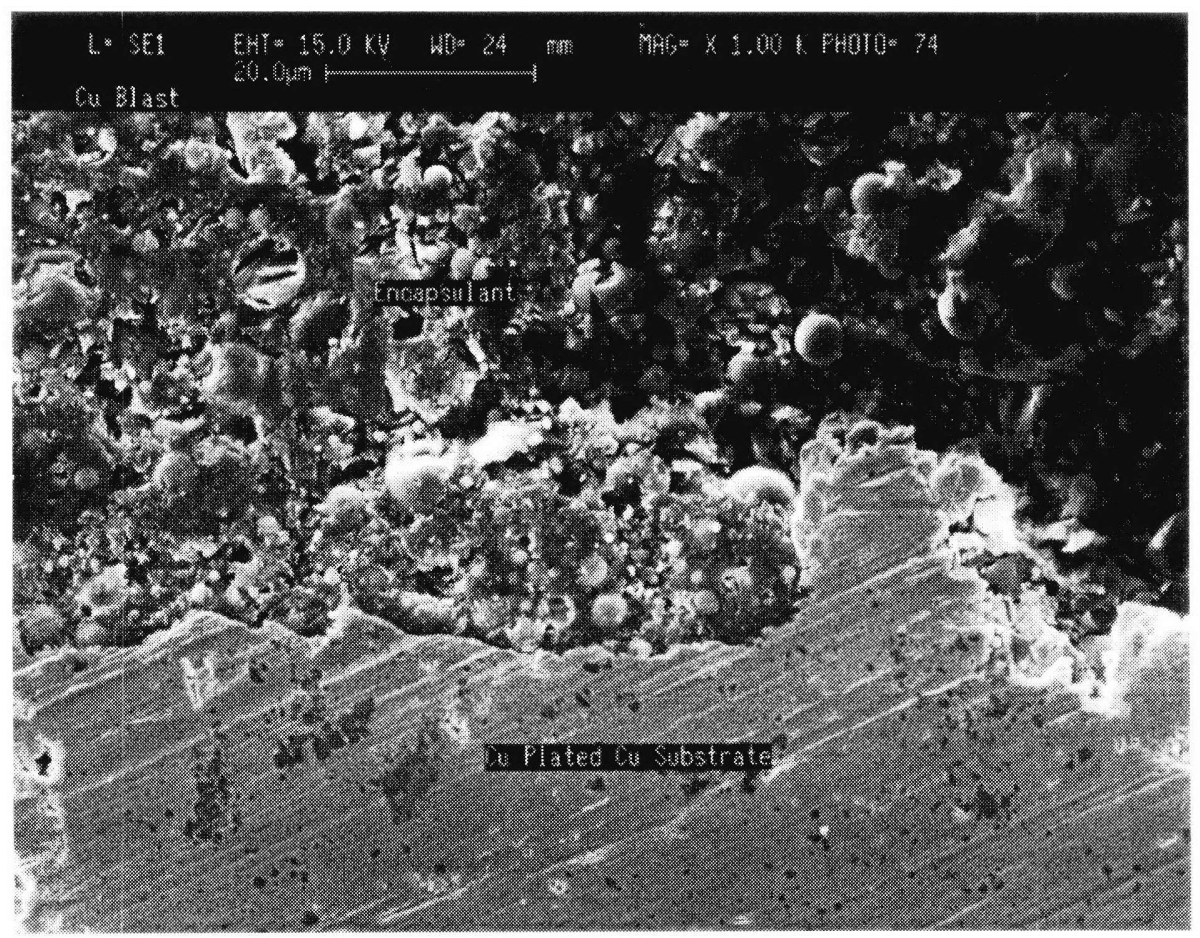

b

Figure 4.4 - SEM micrographs (1000x) of the encapsulant/copper interface in specimens made from a) as plated and b) blast-treated substrates 


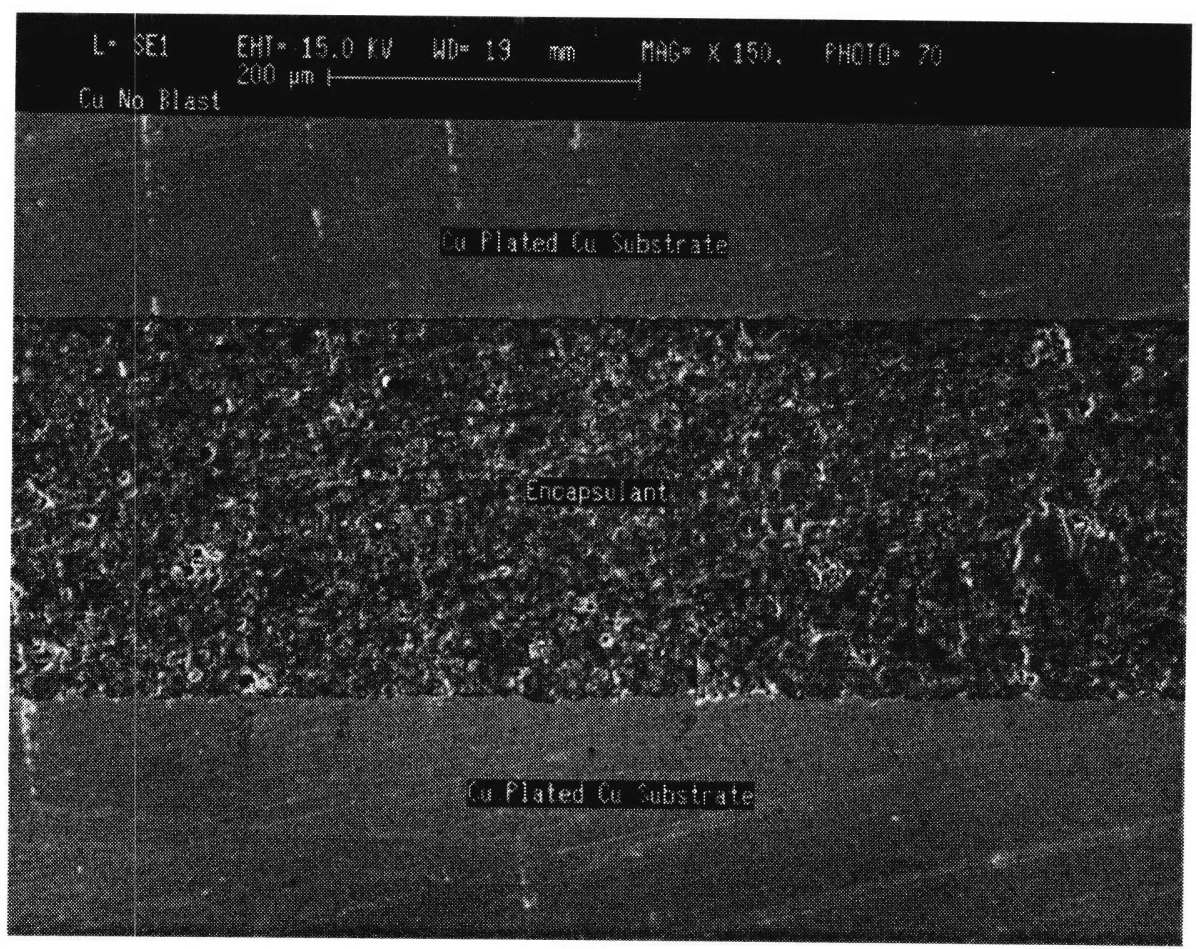

a

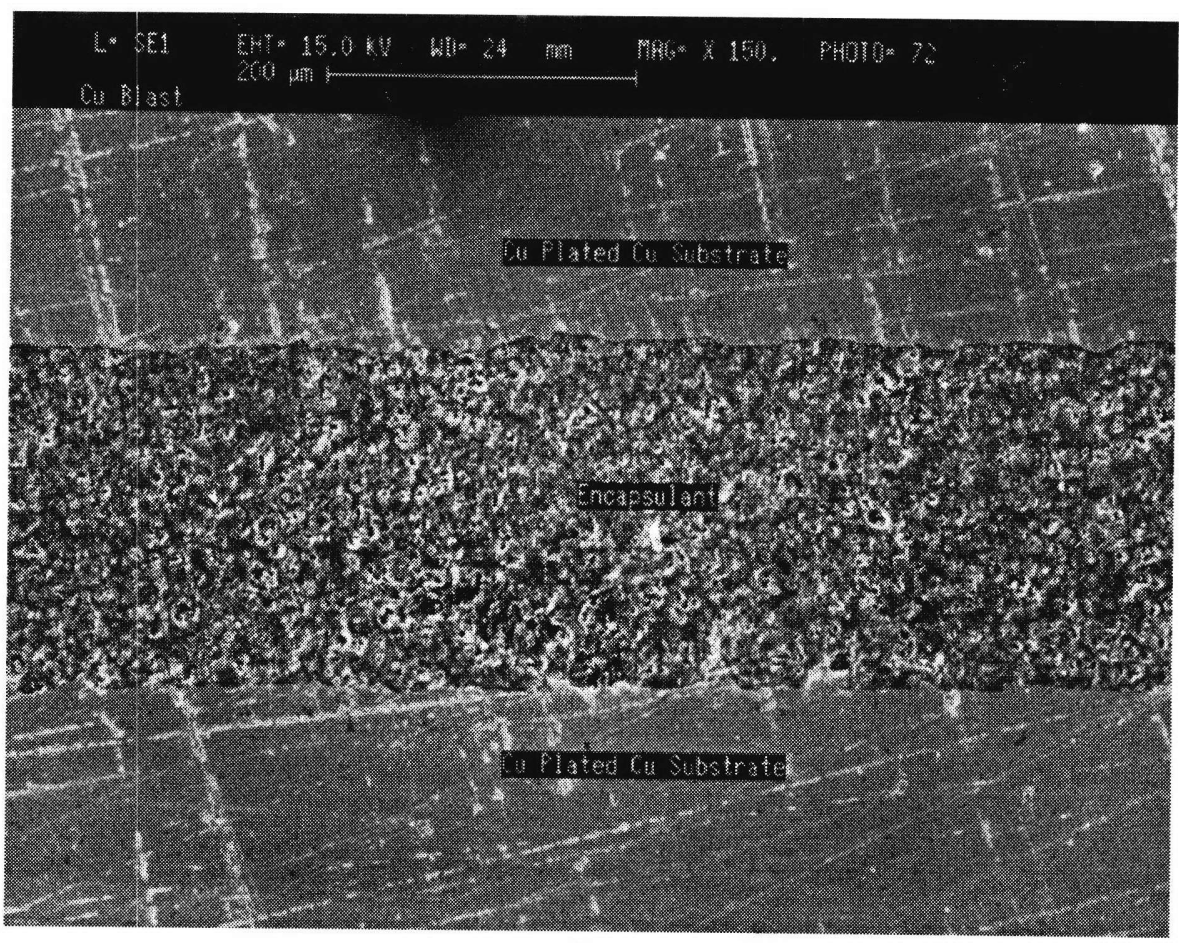

b

Figure 4.5 - SEM micrographs (150x) of the bonding region of specimens made from a) as plated and b) blast-treated copper substrates 


\subsection{Package-level Reliability}

Scanning acoustic microscopy (SAM) was used to detect delamination at the encapsulant/heat slug interface following 1000 cycles of temperature cycle " $\mathrm{B}$ " $\left(-55^{\circ}\right.$ $\left.125^{\circ} \mathrm{C}\right)$. The results are summarized in Table 4.5. Packages built using encapsulant $\mathrm{C}$ showed complete delamination following reliability testing. Materials B and A fared much better, displaying less than $10 \%$ and no delamination, respectively. Based on these results, the intrinsic adhesion at the encapsulant/nickel interface should be least for material $\mathrm{C}$ and greatest for material A, with material B slightly less than A. Note that this trend is opposite the trend predicted by the work of adhesion as calculated by contact angle measurements. Therefore, the experimentally determined work of adhesion does not seem to be an accurate predictor of package performance.

Table 4.5 - Delamination results from package-level reliability testing

\begin{tabular}{|c|c|}
\hline Candidate Encapsulant & $\begin{array}{c}\text { Percent Delaminated Area at the } \\
\text { Encapsulant / Heat Slug Interface }\end{array}$ \\
\hline Material A & $0 \%$ \\
Material B & $<10 \%$ \\
Material C & $100 \%$ \\
\hline
\end{tabular}

\subsection{Fatigue Crack Propagation Experiments}

Strain energy release rates $(G)$ were calculated using both the homogenous solution (eq. 3.11) and the exact solution (eq. 2.24). In all cases the homogenous solution overestimated the exact strain energy release rate by one to three percent. This difference was not considered very significant, but could become more significant as the thickness of the adhesive layer is increased. Nonetheless, all of the strain energy release rate data reported in this chapter were calculated using the exact solution described in section 2.2.4. 


\subsubsection{Failure Mode}

Since subcritical crack growth can occur under static loading conditions, it was necessary to verify that the crack growth mechanism was associated with the cyclic loading, not the maximum stress. Therefore, a test was interrupted during crack propagation in the Paris regime and held at maximum load. After 30 minutes, cyclic loading was resumed. No crack growth occurred during the hold period (see Figure 4.6). Therefore, the subcritical crack growth was due to the cyclic loading.

\section{Crack lenght vs. time showing the effect of a hold} at maximum load

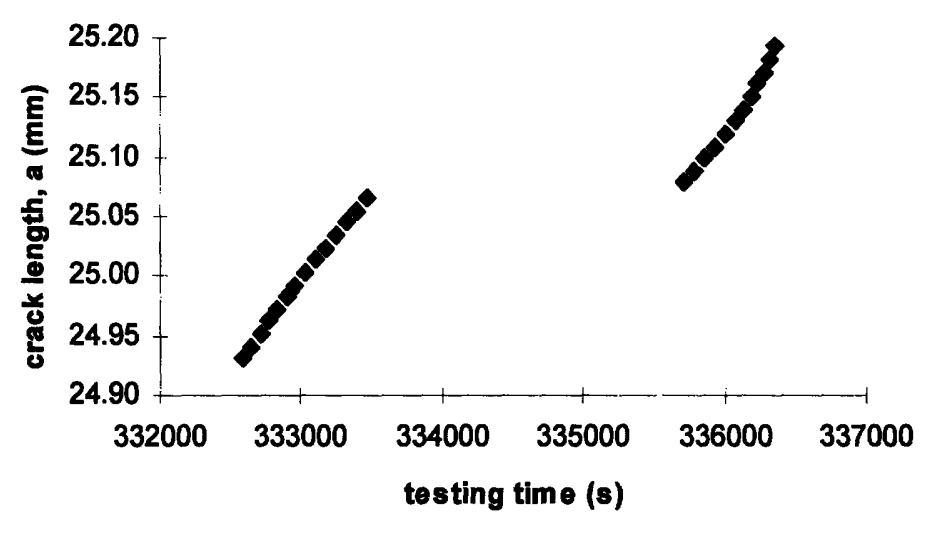

Figure 4.6 - Graph of crack length vs. time showing that no crack growth occurred during a 30 minute hold at maximum load

SEM examination of the fractured surfaces proved that fatigue crack growth progressed along the upper polymer/metal interface. Figure 4.7 shows the crack path for a specimen made from blast-treated copper substrates. Note that crack propagation occurred along the encapsulant/copper interface. A photograph of the two fatigue fracture surfaces of a typical specimen is shown in Figure 4.8. No encapsulant could be detected optically on any of the metal fracture surfaces; therefore all of the failures were considered adhesive in nature. Note that the fatigue crack growth region only extended from $2.5-3.5 \mathrm{~cm}$ on the scale next to the specimen. The fracture surfaces were then examined via SEM, and the results are discussed in section 4.5. In summary, it was verified that all samples failed adhesively due to the action of a cyclic fatigue mechanism. This process will be referred to as interfacial fatigue crack propagation. 


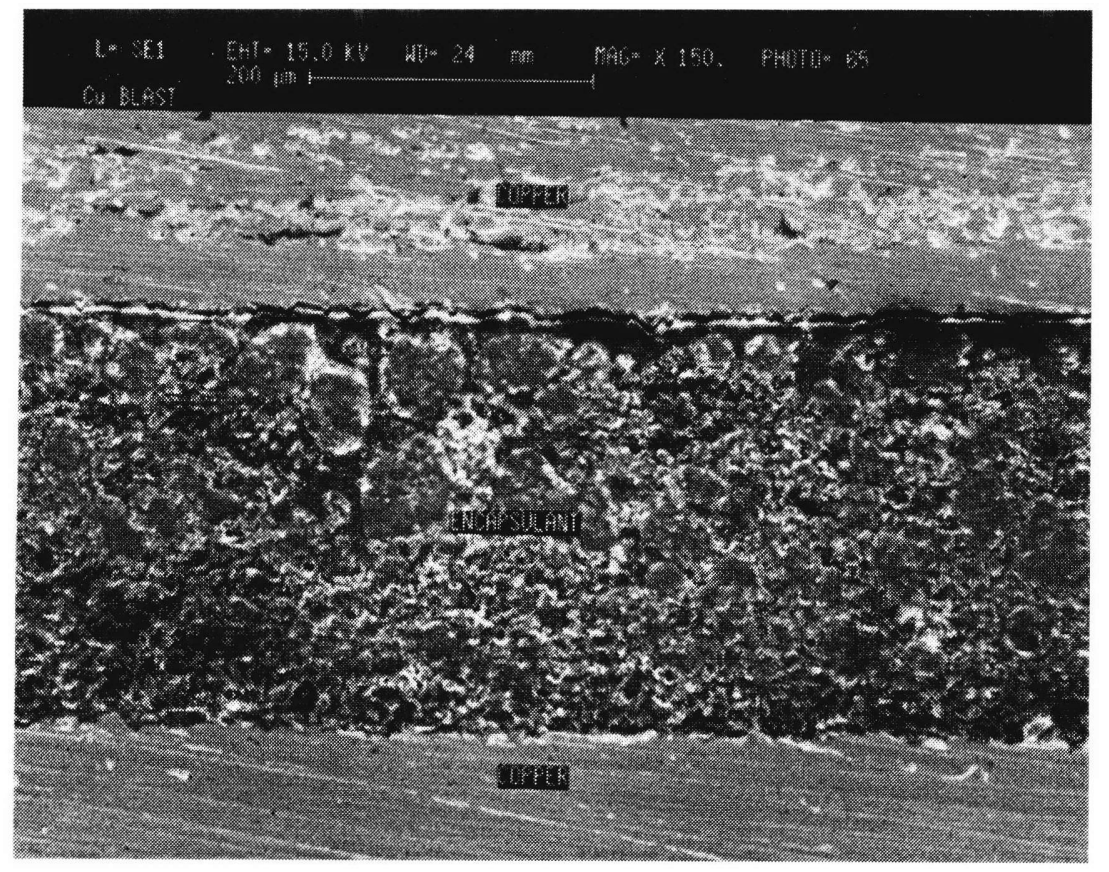

Figure 4.7 - SEM micrograph (150x) showing the crack path along the top encapsulant/copper interface

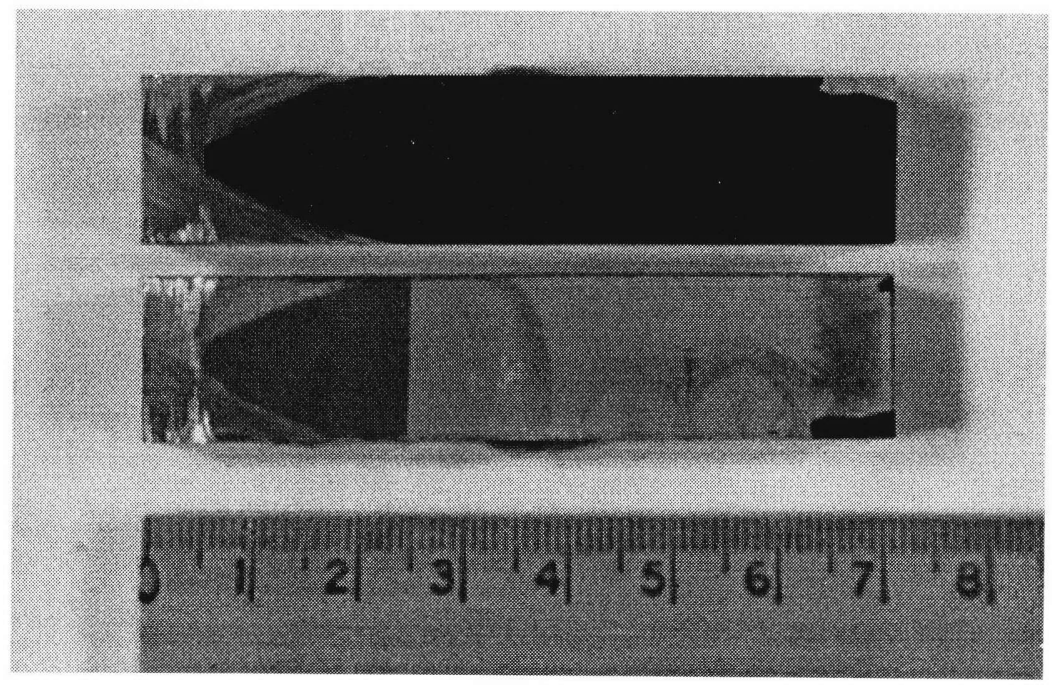

Figure 4.8 - Photograph of typical DCB fracture surfaces 


\subsubsection{Encapsulant Comparison}

The purpose of this phase of the experiment was to determine the interfacial fatigue crack propagation resistance of three different encapsulant materials bonded to nickel substrates in the as-plated condition. The same encapsulants were used to build test packages and were subjected to reliability stressing. Based on the FCP experimental results, conclusions about the fatigue lives of the various systems were made. These conclusions were then compared to the package-level results to see whether interfacial FCP data represented the in-service performance of the materials.

Experimental Results:

Interfacial FCP data were successfully collected on the three candidate encapsulant materials. The crack growth curves are shown in Figure 4.9. All three material systems exhibited a well-defined Paris regime, consistent with equation 2.26. A linear regression was used to calculate, $n$, the slope of the FCP curve in the Paris regime. Near threshold crack growth rates were reached for materials B and C. $\Delta G_{t h}$ was not reached for material $\mathrm{A}$, due to the large amount of testing time spent in the Paris regime. The crack propagated through the entire gage before threshold could be reached. However, based on the trends of the FCP curves, it can be assumed that materials B and A should have similar thresholds, significantly above that of material C. For the purpose of comparison, $\Delta G^{*}$ was defined as the strain energy release rate range required to propagate a crack at a rate of $10^{-4} \mathrm{~mm} / \mathrm{cycle}$. All of these key parameters $\left(n, \Delta G_{t h}, \Delta G^{*}\right)$ are summarized in Table 4.6.

Table 4.6 - Summary of key interfacial FCP parameters for candidate encapsulants on as-plated nickel

\begin{tabular}{|c|c|c|c|}
\hline Encapsulant & $\Delta G_{t h}\left(\mathrm{~J} / \mathbf{m}^{2}\right)$ & $\begin{array}{c}n, \text { slope of linear } \\
\text { portion of } \log d a / d N \\
\text { vs. } \log \Delta G \text { curve }\end{array}$ & $\begin{array}{c}\Delta G^{*}\left(\mathbf{J} / \mathbf{m}^{2}\right) \text { at a } \\
\text { crack growth rate } \\
\text { of } 10^{-4} \mathbf{~ m m} / \text { cycle }\end{array}$ \\
\hline Material A & $\sim 4.0$ & 3.4 & 14.5 \\
\hline Material B & 4.0 & 5.0 & 7.9 \\
\hline Material C & 2.0 & 9.3 & 3.1 \\
\hline
\end{tabular}


Encapsulant on Ni - Interfacial FCP Comparison

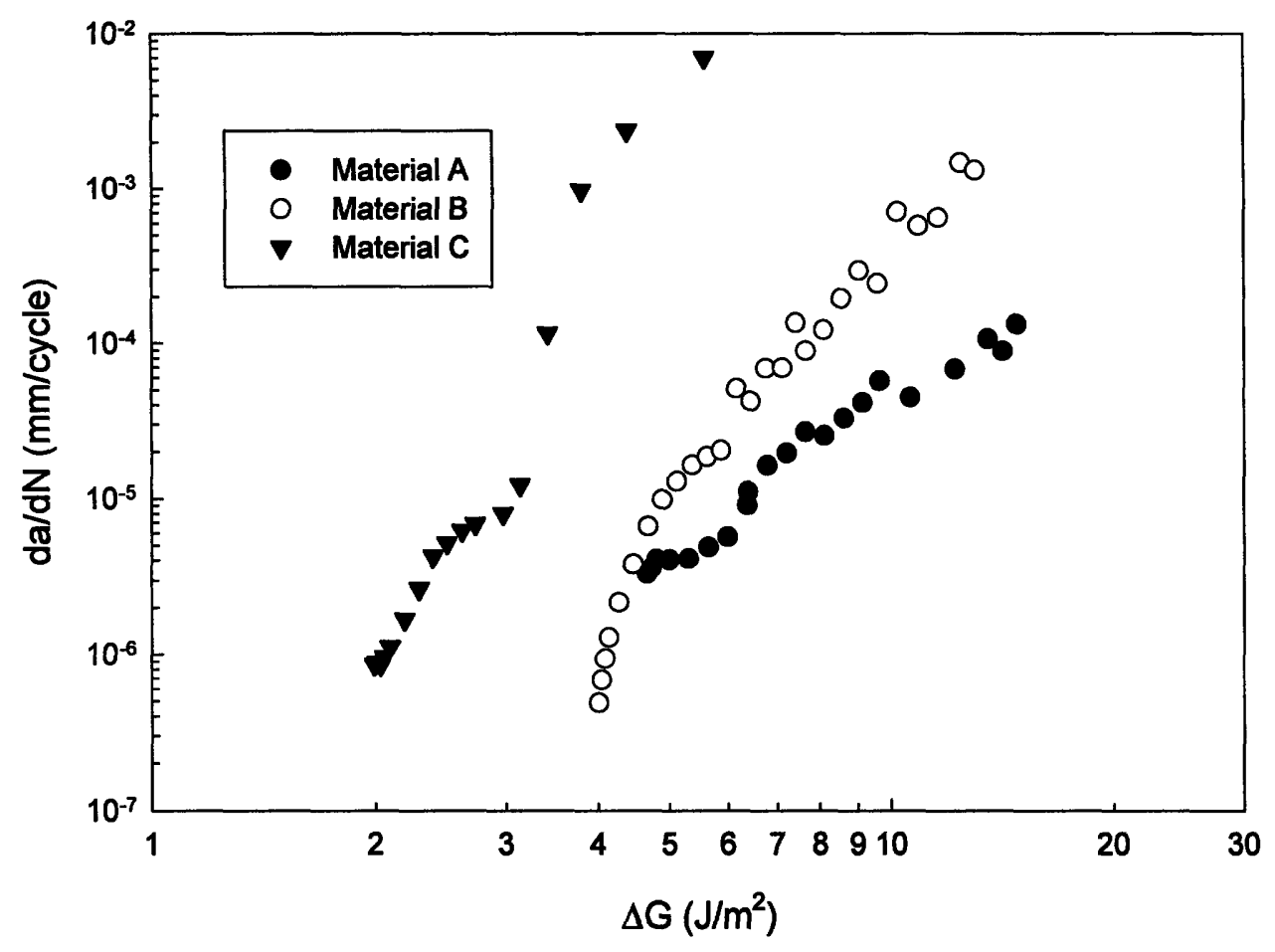

Figure 4.9 - Interfacial FCP data for three candidate encapsulants bonded to as-plated nickel

Among the three materials tested, material $\mathrm{C}$ clearly exhibited the worst interfacial FCP resistance in terms of both threshold strain energy release rate and crack growth rate sensitivity to changes in driving force $(n)$. The brittleness of this system was demonstrated by the relatively large value of the slope in the Paris regime. The entire range of tested $\Delta G$ only extended from $2-5 \mathrm{~J} / \mathrm{m}^{2}$, compared to a range of $4-14 \mathrm{~J} / \mathrm{m}^{2}$ in the case of materials B or A. Materials B and A exhibited similar near threshold behavior, but material B displayed a much higher slope in the Paris regime. The crack growth rate in the material $\mathrm{A} /$ nickel system was therefore much less sensitive to changes in $\Delta G$. If the linear trend is extended into the regime of unstable crack growth (as $G_{\max } \rightarrow$ $G_{c}$ ), material A would exhibit a fracture toughness approximately twice that of material B. 


\section{Fatigue-Life Implications:}

The FCP characteristics of these systems have many implications for the service lives of the interfaces under cyclic loading. Assuming that a crack-like flaw exists, the service life of the component is determined by the time required for the crack to grow to a critical length, at which point it propagates catastrophically. If the loading conditions at the crack tip result in a stress state below the FCP threshold, then the service life is essentially infinite; no failure will occur via a fatigue mechanism. If the FCP threshold is exceeded, then the fatigue-life estimate is based on the integration of equation 2.26. Recognizing that $\Delta G$ is a function of the crack length, equation 2.26 becomes:

$$
\frac{d a}{d N}=B[\Delta G(a)]^{n}
$$

Thus, as the crack length increases, $G$ also increases, which leads to an even higher growth rate. Thus, the number of cycles to failure, $N_{f}$, is found by integration:

$$
\int_{f}^{N_{f}} B d N=\int_{a_{i}}^{a_{c}} \frac{d a}{[\Delta G(a)]^{n}}
$$

where $a_{i}$ is the initial crack length and $a_{c}$ is the critical crack length. Therefore, $N_{f}$ is given by:

$$
N_{f}=\frac{1}{B} \int_{a_{i}}^{a_{c}} \frac{d a}{[\Delta G(a)]^{n}}
$$

which is highly dependent on $n$, the slope of the FCP curve in the Paris regime.

The low threshold of the material $\mathrm{C}$ system indicated that a crack will begin to propagate under loading conditions that would not cause crack propagation in either the material B or A systems. Furthermore, the steep slope in the Paris regime implied that the crack, once propagating, will grow to a critical length very rapidly, leading to fast fracture. It was interesting to note that the strain energy release rate range necessary to cause unstable crack growth along the material $\mathrm{C} /$ nickel interface was on the order of the threshold in both the material A and B systems. Since the A and B systems had similar thresholds, crack growth will occur under similar conditions for the two materials. However, since material A exhibited a smaller $n$, it should have a longer fatigue life. As seen in Figure 4.9, at the $\Delta G$ necessary for the onset of unstable crack growth in material $B$, the crack growth rate in material A was still stable and on the order of $10^{-4} \mathrm{~mm} / \mathrm{cycle}$. 
Correlation with Package-Level Testing:

The interfacial FCP results were in very good agreement with the package-level temperature cycling tests. After 1000 cycles, material $\mathrm{C}$ was observed to completely delaminate from the nickel heat slug surface, material B exhibited slight delamination, and material A showed no delamination. This suggested that the loading conditions were severe enough to induce noticeable crack growth in material B, but not in material A. Since the FCP results predicted that cracks would grow more slowly at the material A interface, this was a reasonable result. Furthermore, if the package experienced conditions severe enough to cause crack growth at the material B interface, the FCP data indicates that material $\mathrm{C}$ should have failed completely, which it did. Therefore, the interfacial FCP data appeared to be a good predictor of delamination performance of actual package structures. Had the temperature cycling been extended beyond 1000 cycles, it would have been interesting to see if the trend predicted by the FCP data continued for the material B and material A packages. However, due to demands on the reliability testing facilities, this was not possible.

Correlation with Work of Adhesion:

Based on the work of adhesion as calculated from contact angle measurements, one would expect material $\mathrm{C}$ to exhibit the best adhesion, material $\mathrm{A}$ the worst, and material B in between. The trend found from interfacial FCP experiments was opposite the trend predicted by work of adhesion. Therefore, the work of adhesion does not seem to be a reliable indicator of interfacial FCP resistance. There are two possible reasons for this. First, the surface energies calculated from the contact angle measurements on cured polymer specimens may not be representative of the material in the adhesive joint. This was discussed in Section 4.1. A second possibility is that there are other energydissipating processes that occur during FCP that obscure the effect of work of adhesion. Since the observed threshold strain energy release rates were on the order of $1 \mathrm{~J} / \mathrm{m}^{2}$, and the calculated $W^{a}$ was on the order of $0.1 \mathrm{~J} / \mathrm{m}^{2}$, this is a very likely explanation also. Comparison with Bulk FCP and Fracture Behavior:

The interfacial FCP data reported here indicate that the fatigue resistance of the encapsulant/metal interface is much less than the fatigue resistance of either the bulk 
encapsulant or the bulk metal. Figure 4.10 shows the FCP curves for the material B/asplated nickel interface, a bulk encapsulant, a typical aluminum alloy, and a typical steel. The FCP resistance of the metal/polymer interface is an order of magnitude less than the bulk encapsulant, and two orders of magnitude less than a typical metal. This indicates that in these polymer/metal components, the interface will fail via a FCP mechanism long before either of the bulk materials. Furthermore, previous work on the mixed mode fracture of polymer/metal interfaces found in microelectronic packaging reported the mode I fracture toughness to be on the order of $100 \mathrm{~J} / \mathrm{m}^{2}$ [4]. Therefore, interfacial fatigue crack propagation occurred at $G$ levels one to two orders of magnitude below those required for static fracture.

FCP Comparison - bulk metals, bulk encapsulant, and encapsulant/Ni interface

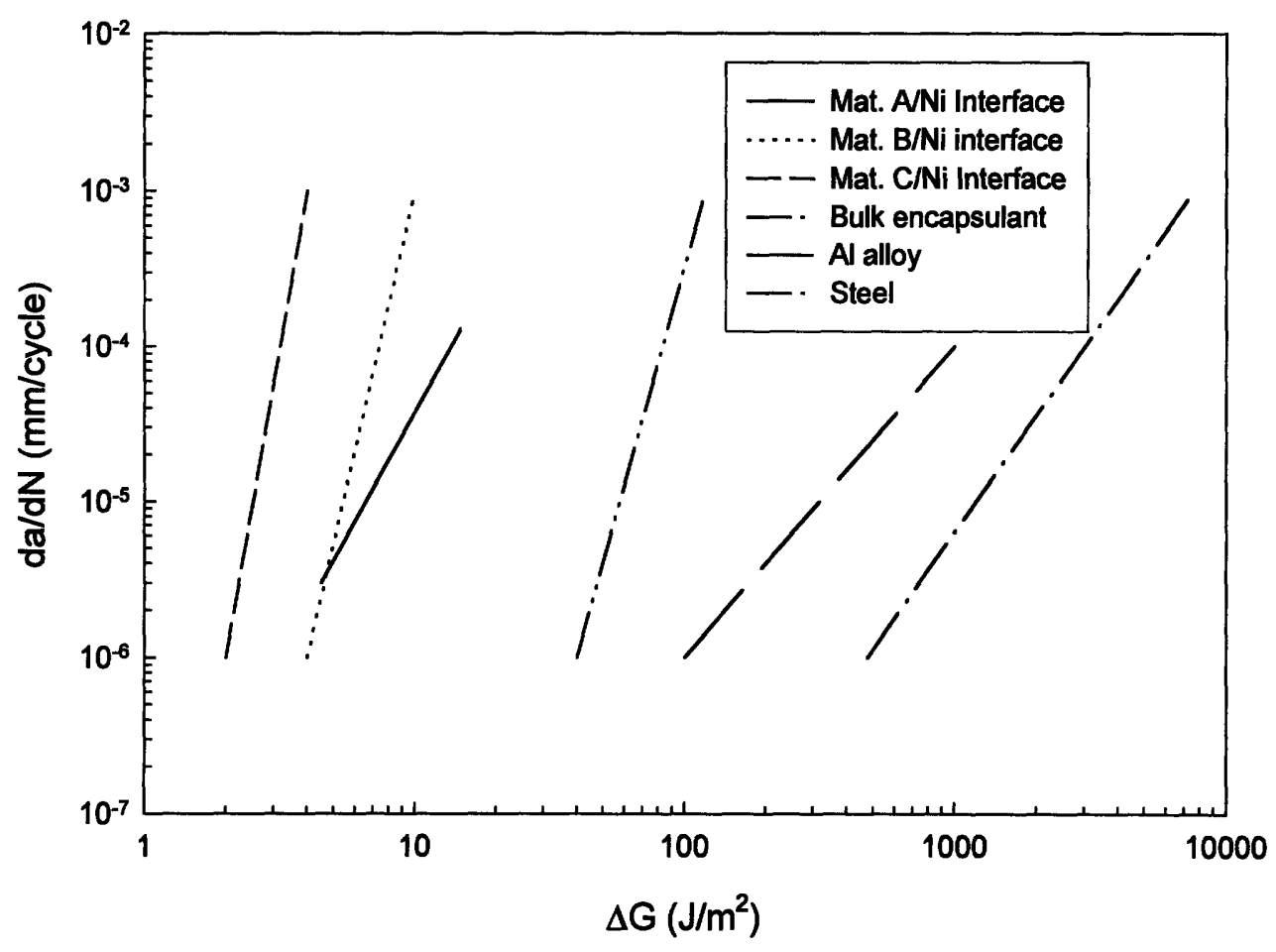

Figure 4.10 - FCP trends for encapsualnt/metal interfaces, bulk encapsulant [3], and bulk metals [38] 


\subsubsection{Effect of Substrate Plating Metal and Surface Roughness}

The purpose of this phase of the experimental work was to alter the properties of the interface (surface roughness and plating metal) in order to gain an understanding of interfacial fatigue crack propagation mechanisms. The experimental results will be presented first, followed by a discussion of the correlation of FCP data with thermodynamic work of adhesion.

Experimental Results:

Interfacial FCP data were successfully collected on specimens made from material $\mathrm{B}$ encapsulant on $\mathrm{Ni}$ as-plated, $\mathrm{Ni}$ blast-treated, $\mathrm{Cu}$ as-plated, and $\mathrm{Cu}$ blast-treated substrates. The crack growth curves may be seen in Figure 4.11 and 4.12. Again, all four material systems exhibited a Paris regime, and a fatigue crack propagation threshold. The key FCP parameters defined in section 4.3.2 were also calculated for these material systems and are shown in Table 4.7.

Table 4.7 - Summary of key interfacial FCP parameters for Material B encapsulant on various substrates

\begin{tabular}{|c|c|c|c|c|}
\hline Substrate & $\Delta G_{t h}\left(\mathrm{~J} / \mathrm{m}^{2}\right)$ & $\begin{array}{c}n \text {, slope of } \\
\text { linear portion } \\
\text { of } \log d a / d N \text { vs. } \\
\log \Delta G \text { curve }\end{array}$ & $\begin{array}{c}\Delta G^{*}\left(\mathrm{~J} / \mathrm{m}^{2}\right) \text { at a } \\
\text { crack growth } \\
\text { rate of } 10^{-4} \\
\text { mm/cycle }\end{array}$ & $\begin{array}{c}\text { Relative } \\
\text { roughness of } \\
\text { substrate, } R\end{array}$ \\
\hline $\begin{array}{c}\text { Nickel as- } \\
\text { plated }\end{array}$ & 4.0 & 5.0 & 7.9 & 22.3 \\
\hline $\begin{array}{c}\text { Nickel blast- } \\
\text { treated }\end{array}$ & 5.2 & 4.2 & 14.6 & 77.8 \\
\hline $\begin{array}{c}\text { Copper as- } \\
\text { plated }\end{array}$ & 3.2 & 3.5 & 9.5 & 10.3 \\
\hline $\begin{array}{c}\text { Copper blast- } \\
\text { treated }\end{array}$ & 6.2 & 4.3 & 15.0 & 90.3 \\
\hline
\end{tabular}


Material $\mathrm{B}$ on $\mathrm{Ni}$ - as-plated vs. blasted

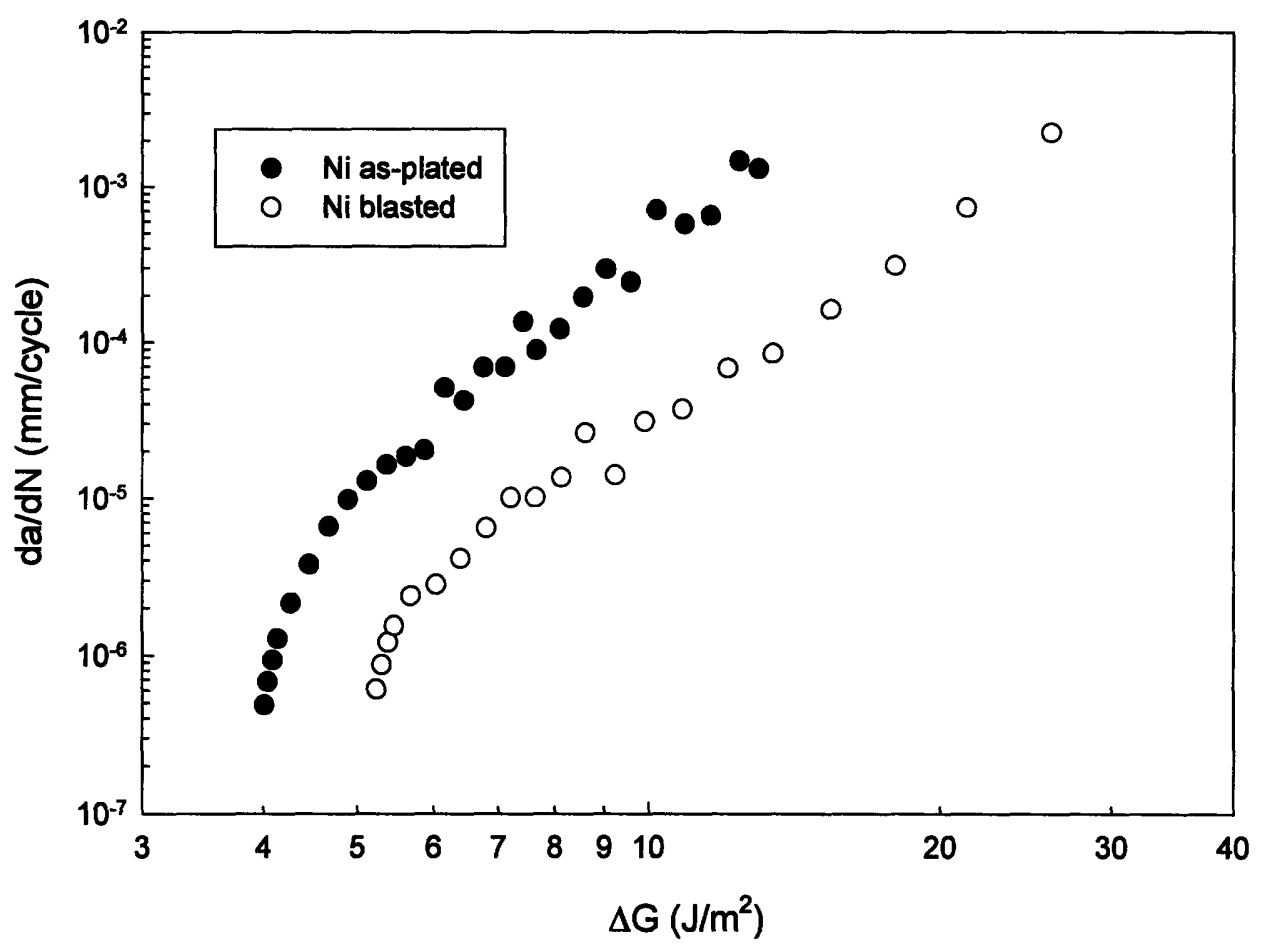

Figure 4.11 - Interfacial FCP data for Material B encapsulant bonded to as-plated and blast-treated nickel

For each plating metal, roughening the surface increased the overall interfacial fatigue crack propagation resistance, both at threshold levels, and at higher crack growth rates. For nickel, inducing more roughness not only increased the $\Delta G_{t h}$, it led to a flatter slope in the Paris regime. This would indicate that the toughening mechanism was not confined to the threshold region, but was active in all FCP regimes. For the copper specimens, the rougher interface exhibited a much larger $\Delta G_{t h}$, but the difference in growth rates at higher levels of $\Delta G$ became less. That is, the FCP resistances at high growth rates were similar. This indicated that the toughening mechanism(s) was more dominant at lower growth rates for the copper systems. These implications will be discussed in section 4.5. 
Material $\mathrm{B}$ on $\mathrm{Cu}$ - as-plated vs. blasted

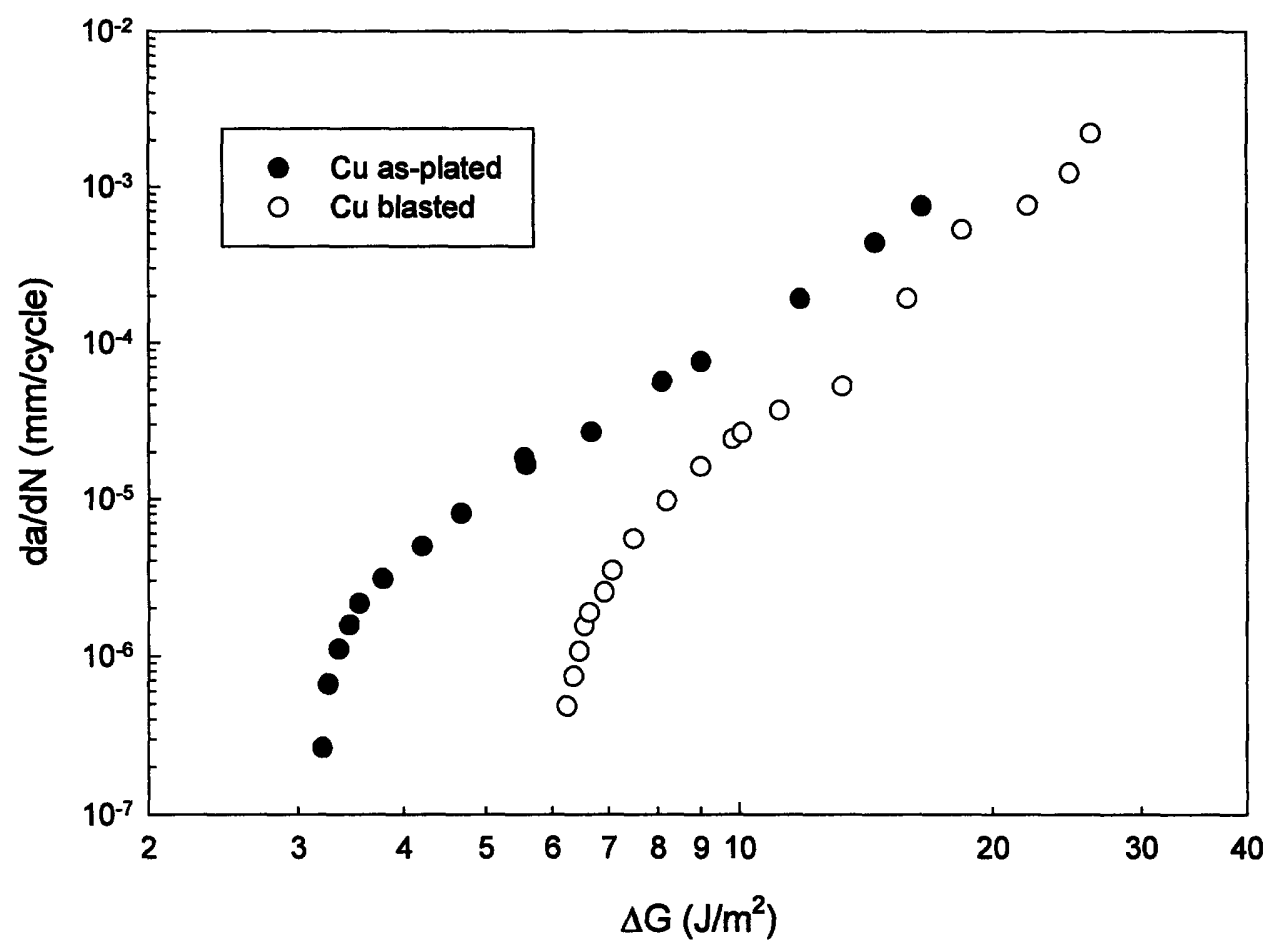

Figure 4.12 - Interfacial FCP data for Material B encapsulant bonded to as-plated and blast-treated copper

\section{Correlation with Work of Adhesion:}

The work of adhesion did not reliably predict interfacial FCP resistance.

However, there was some limited success. For the as-plated substrates, the work of adhesion for nickel $\left(82.41 \mathrm{~mJ} / \mathrm{m}^{2}\right)$ was slightly higher than for copper $\left(74.69 \mathrm{~mJ} / \mathrm{m}^{2}\right)$. The same trend was observed in threshold strain energy release rate. However, the work of adhesion for the blasted nickel system was well below the other three, but the blasted nickel system's FCP resistance was actually higher. This supports the notion that the contact angle measurement technique is not well-suited for examining rough surfaces. Since there was some correlation between FCP resistance and $W^{a}$ for the smoother 
surfaces, the contact angle measurement technique may still be useful, but only for probing very smooth surfaces.

\subsubsection{Fractography}

As mentioned previously, all of the fatigue specimens were found to fail adhesively at the encapsulant/metal interface. Figure 4.13 shows the metal fatigue-fracture surface for material $B$ on the blasted nickel substrate. Even at a magnification of $1250 \mathrm{x}$, no encapsulant could be detected on the metal surface. Similar results were found for the other material systems.

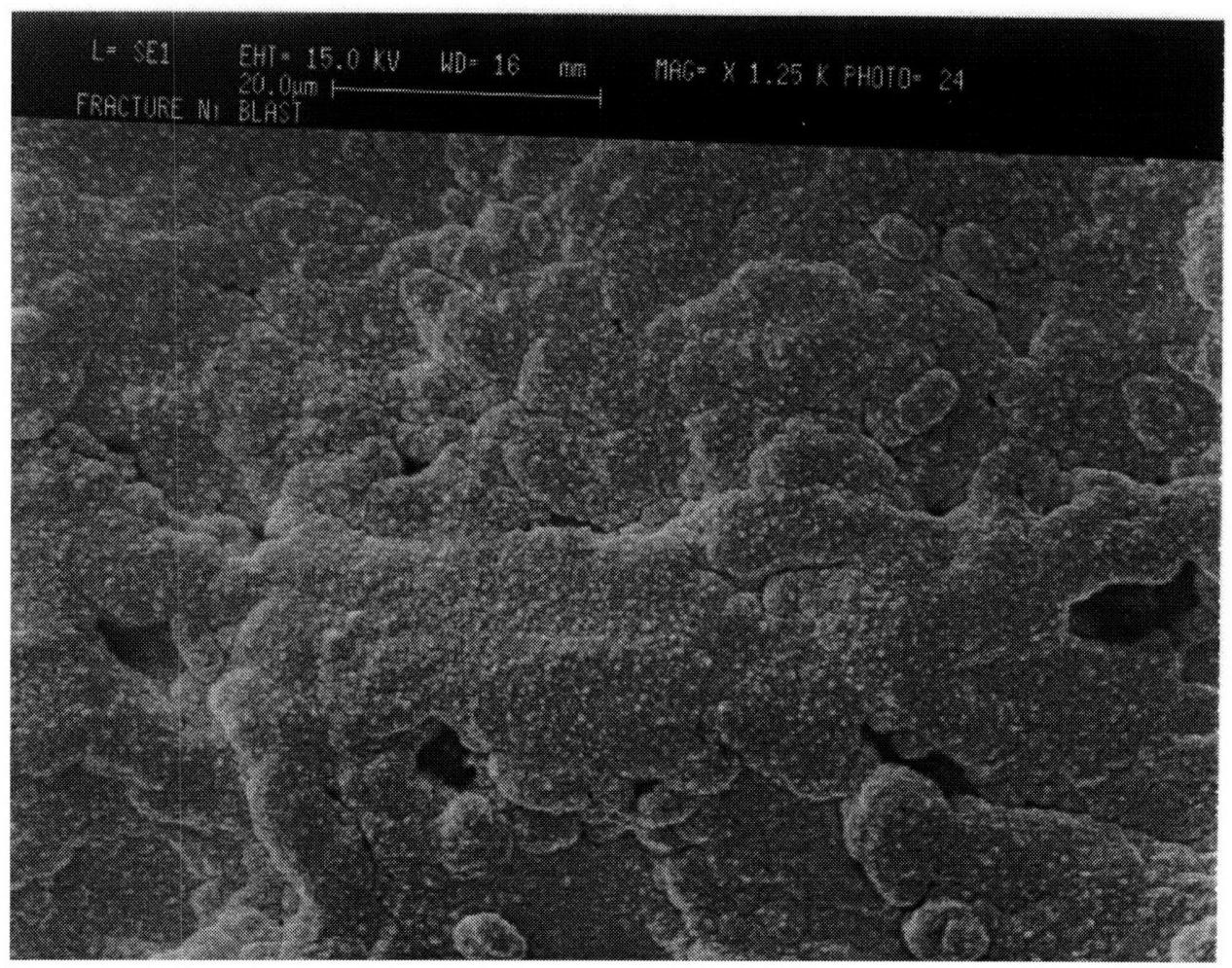

Figure 4.13 - SEM micrograph (1250x) showing metal fatigue-fracture surface for material $B$ on blasted nickel substrate

Microscopic analysis of the encapsulant fatigue-fracture surfaces was difficult for two reasons. First, the presence of such a large volume fraction of filler particles on the fractured encapsulant surface tended to obscure any detail. The same problem was encountered during analysis of the fatigue-fracture surfaces of bulk encapsulant FCP 
specimens in a previous study [3]. Figure 4.14 shows the encapsulant fatigue-fracture surface of a specimen made from material B on as-plated copper. Note the lack of detail on the fracture surface and the absence of typical fatigue-fracture features, such as striations. No fatigue striations were observed on any of the fatigue-fracture surfaces.

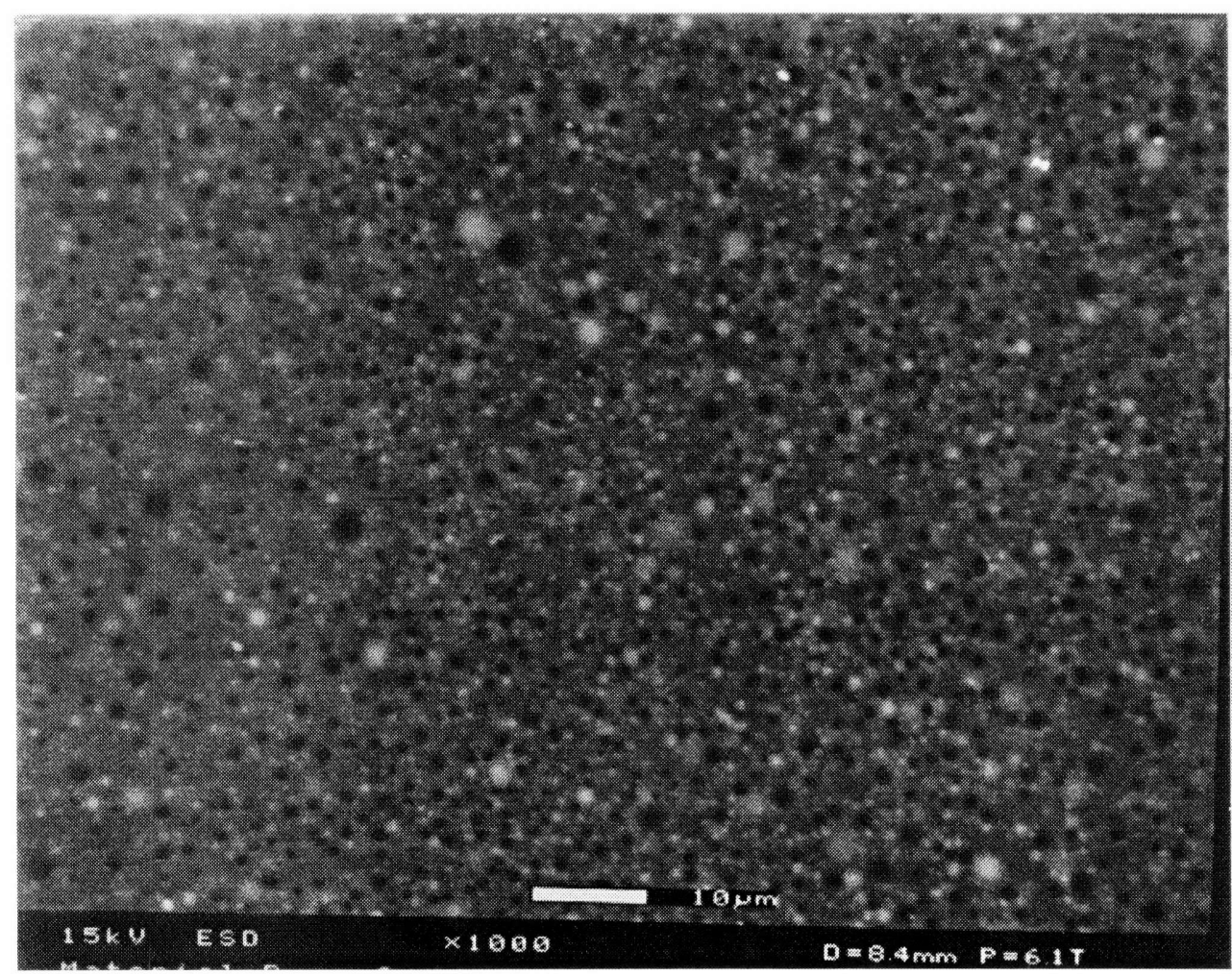

Figure 4.14 - SEM micrograph (1250x) showing encapsulant fatigue-fracture surface for material B on as-plated copper substrate

Second, due to the intimate contact of the encapsulant with the metal, the encapsulant fracture surfaces were basically "mirror images" of the metal substrate surfaces. With the exception of the as-plated copper specimens all of the metal substrate surfaces were very rough. Therefore, any detail on the encapsulant fatigue-fracture surface was also obscured by the topography of the metal plating. Figure 4.15 shows the encapsulant fatigue-fracture surface of a specimen made from material B on blast-treated nickel. The only discernible detail is the topography of the metal surface to which the encapsulant was bonded. 


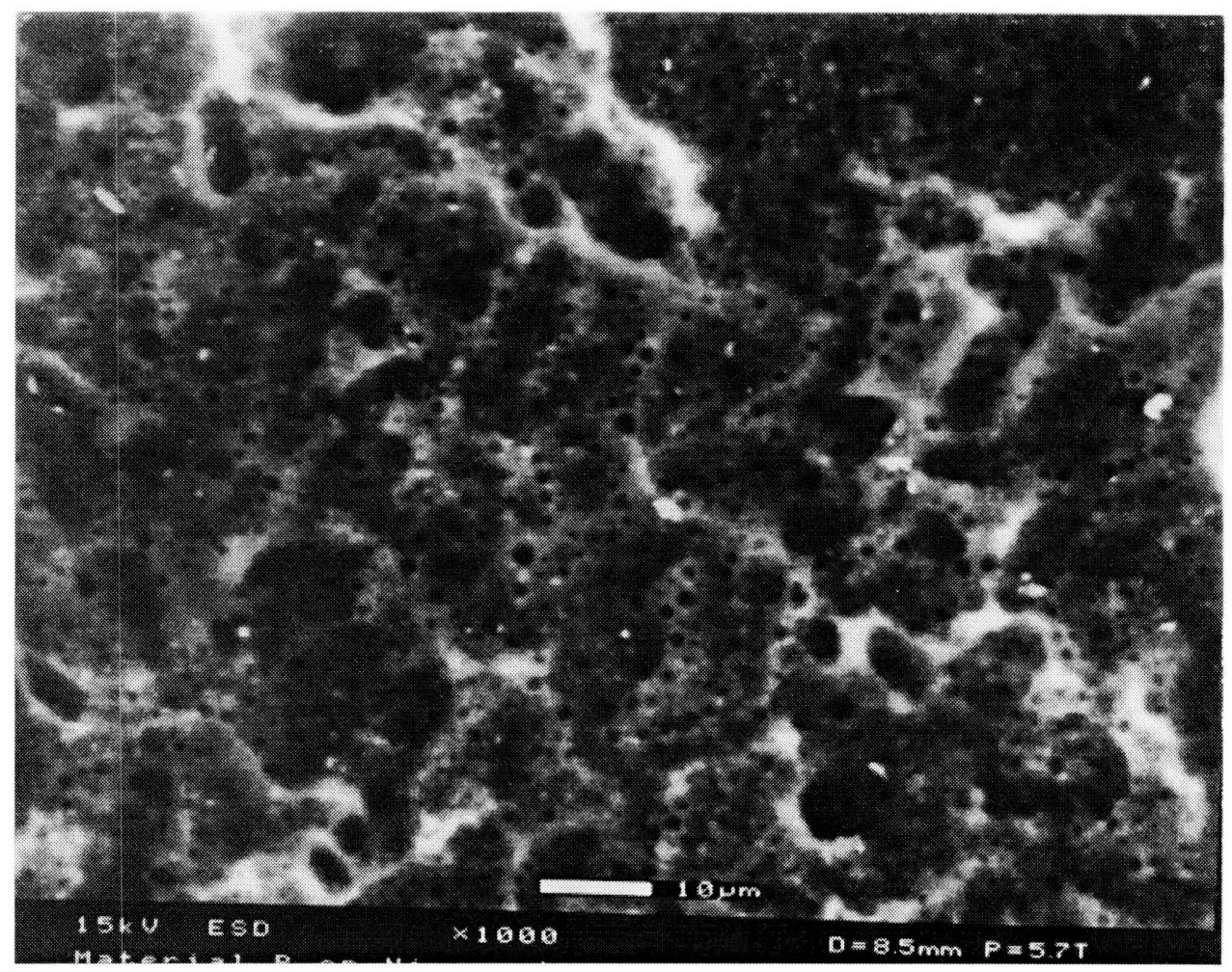

Figure 4.15 - SEM micrograph (1000x) showing encapsulant fatigue-fracture surface for material B on blasted nickel substrate

The only other interesting feature observed was on the fatigue-fracture encapsulant surface from material A bonded to as-plated nickel substrate. Metal particles were found embedded in the encapsulant surface (see Figure 4.16). This suggested that unlike all the other specimens, where crack propagation involved only debonding at the interface, crack propagation in this specimen involved some fracturing of metal asperities embedded in the polymer material. This explains the very high interfacial FCP resistance of the material system. 


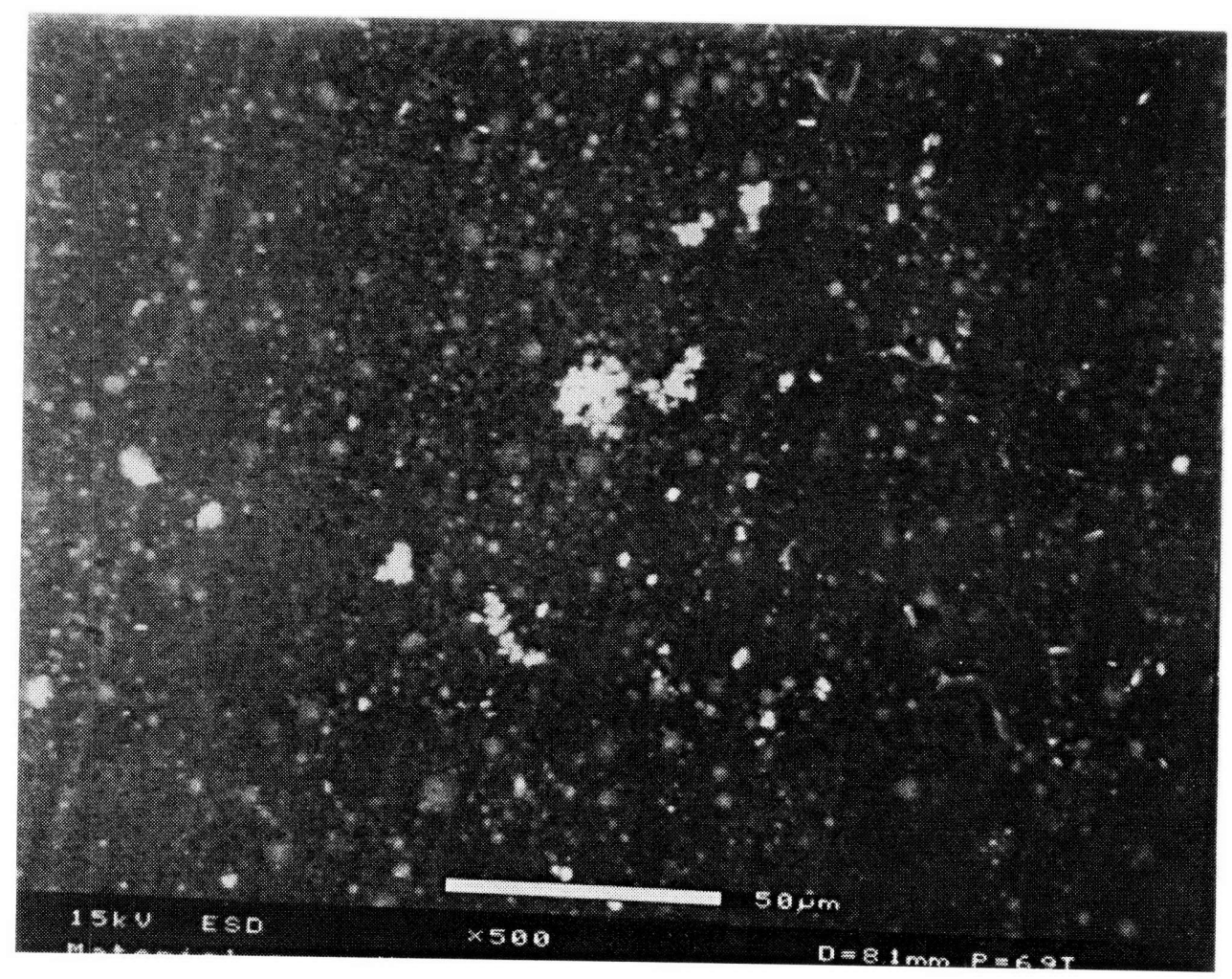

Figure 4.16 - SEM micrograph (500x) showing encapsulant fatigue-fracture surface for material A on as-plated nickel substrate

\subsection{Shielding Mechanisms}

On the basis of the results obtained from FCP experiments and subsequent fractography, one can postulate some shielding mechanisms associated with crack growth at an interface. The mechanisms postulated here include interfacial crack deflection, roughness induced crack closure, and bridging by metal asperities.

\subsubsection{Interfacial Crack Deflection}

For both the copper and nickel systems, increasing the interface roughness resulted in improved FCP resistance in all crack growth rate regimes. As mentioned in section 2.3.2, one shielding mechanism that has been observed to have this type of effect in bulk materials is crack deflection. In bulk materials, the crack may be deflected from a planar growth profile by impurities, second phases, or other microstructural features. An 
interfacial crack is constrained to propagate along the interface; therefore, any nonplanarity or roughness in the interface will result in crack-tip shielding.

Suresh [60] has modeled the effect of crack-tip deflection on fatigue driving force, assuming the geometry shown in figure 4.17 . The effective fatigue driving force $\left(\Delta k_{\text {eff }}\right)$ can be related to the applied stress intensity range $\left(\Delta K_{I}\right)$ through

$$
\Delta k_{e f f}=\frac{D \cos ^{2}(\theta / 2)+S}{D+S} \Delta K_{I}
$$

where $\theta$ is the deflection angle, $D$ is the deflected distance, and $S$ is the undeflected distance. Thus, the shielding effect is minimal when $D / S$ is small, but becomes more pronounced when $D / S$ gets larger. Recognizing that the parameter $D / S$ is similar to the relative roughness parameter $R$ (defined in section 3.2.2), then the shielding effect should increase with $R$. Qualitatively, this trend was observed in both the $\mathrm{Ni}$ and $\mathrm{Cu}$ systems. Suresh also analyzed the effect of closure via a parameter $\chi$, which represents the ratio of in-plane displacement to normal displacement. Larger values of $\chi$ imply greater levels of closure. The effective fatigue driving force, when deflection and closure are present is given by:

$$
\Delta k_{e f f}=\left\{\frac{D \cos ^{2}(\theta / 2)+S}{D+S}\right\} \cdot\left\{1-\left[\frac{\chi \tan \theta}{1+\chi \tan \theta}\right]^{1 / 2}\right\} \Delta K_{I} .
$$

Deflections in crack path also cause apparent changes in crack propagation rates [60]. The crack growth rate of a linear crack, represented by $(d a / d N)_{L}$, may be related to the average propagation rate of a tilted crack, at equal values of $\Delta k_{\text {eff }}$ by:

$$
\left(\frac{d a}{d N}\right)=\left\{\frac{D \cos \theta+S}{D+S}\right\}\left(\frac{d a}{d N}\right)_{L}
$$

when crack length is measured along the Mode I direction. 


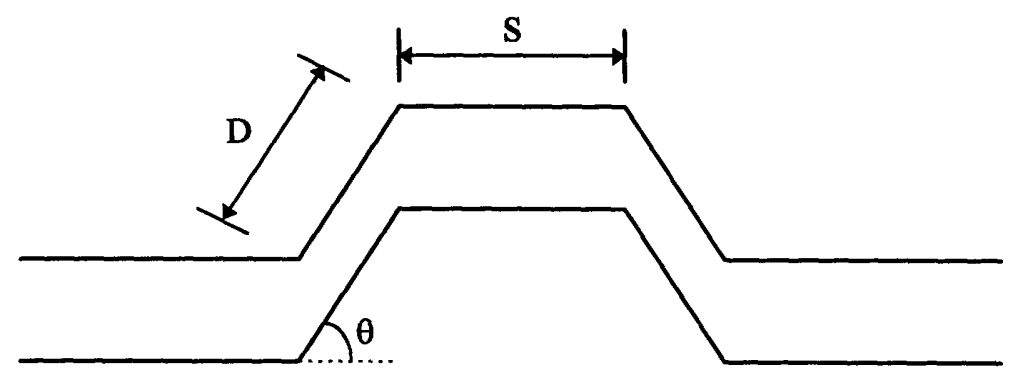

a

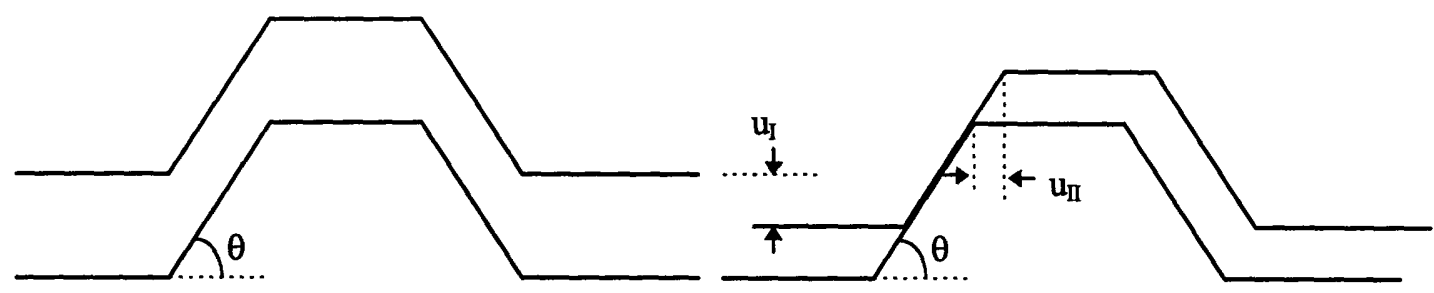

b

Figure 4.17 - a) Schematic drawing of a crack with periodic tilts

b) Schematic drawing of a deflected crack in fully opened condition at the peak load of the fatigue cycle (on the left) and relative mismatch between the fracture surfaces at the point of first contact during unloading (on the right)

Thus, given FCP data for an ideally straight crack $(\theta=0, D / D+S=0)$, equations 4.6 and 4.7 can be used to predict the measured FCP response under various conditions of deflection and closure. Among all the material systems tested, as-plated copper had the smoothest interface; hence, it will be considered to be a "straight" crack for the purpose of this analysis. Figure 4.18 shows the predicted crack propagation rates along the encapsulant-copper interface under various conditions of deflection (as quantified by $D / D+S$ ) and closure (represented by $\chi$ ). An average $\theta=45^{\circ}$ was assumed. It can be seen that increasing the values of the parameters $D / D+S$ and $\chi$ shift the fatigue crack growth curve to the right of that corresponding to the un-deflected crack, leading to an apparent increase in FCP resistance. 
The predictions based on the data from as-plated copper interfaces can be used to analyze the data from the rougher, blast-treated copper specimens. Figure 4.19 shows the FCP curves for the as-plated and blast-treated copper specimens, along with predicted FCP curves for $\chi=0.05$ and 0.10 , assuming $\theta=45^{\circ}$ and $D / D+S=0.75$. For high growth rates, the data from the blast-treated specimen approach the prediction for a deflected crack in the absence of closure. At lower growth rates, the data approach the prediction for a deflected crack with slight closure, given by $\chi=0.05$. Since crack-tip opening displacements are large at high growth rates, closure is not expected. However, at nearthreshold growth rates, crack-tip opening displacements are very small, and closure is more likely. These trends are clearly seen when the experimental data is compared to the model predictions. These trends are also similar to trends seen for near threshold fatigue crack growth in aluminum alloys [38]. For aluminum alloys, $\chi$ was found to be approximately $0.1-0.2$. It was also found that the maximum increase in closure occurred at small $\chi$. Further increases in $\chi$ lead to smaller increases in closure. These trends can also be seen in Figure 4.18. 
Deflection and Closure Effects for $D / D+S=0.5$

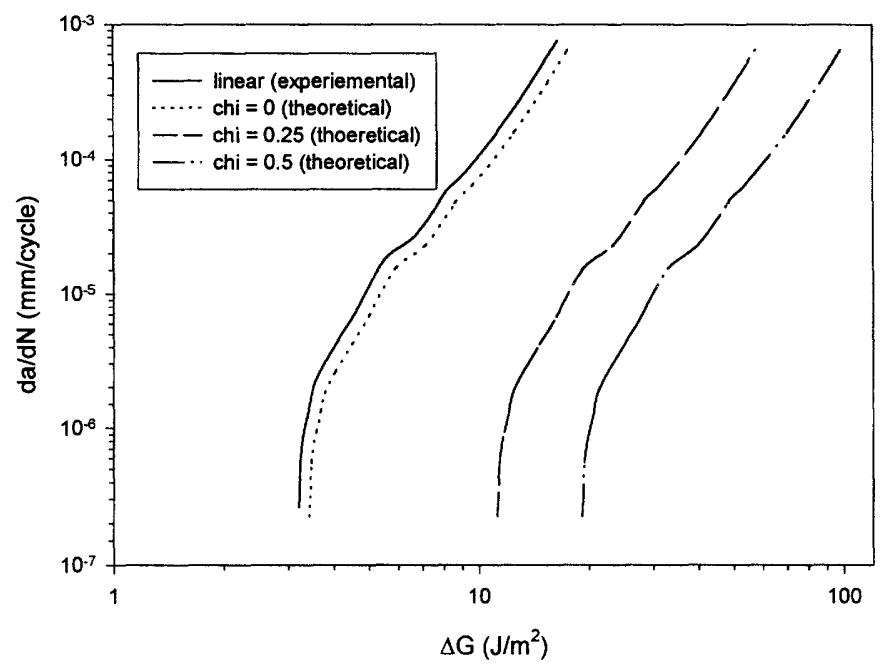

a

Deflection and Closure Effects for $\mathrm{D} / \mathrm{D}+\mathrm{S}=0.75$

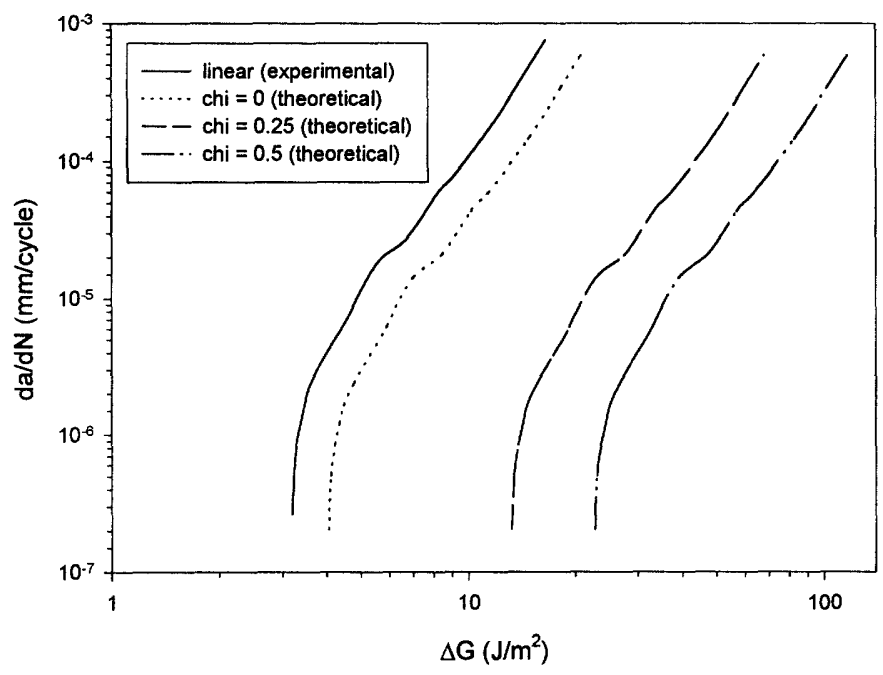

b

Figure 4.18 - Predicted FCP rates for deflected cracks as functions of the closure factor $\chi$ for $\theta=45^{\circ}$ and $D / D+S$ values of (a) 0.5 and (b) 0.75 


\section{Deflection and Closure Predictions vs. Experiment \\ (for $D / D+S=0.75$ )}

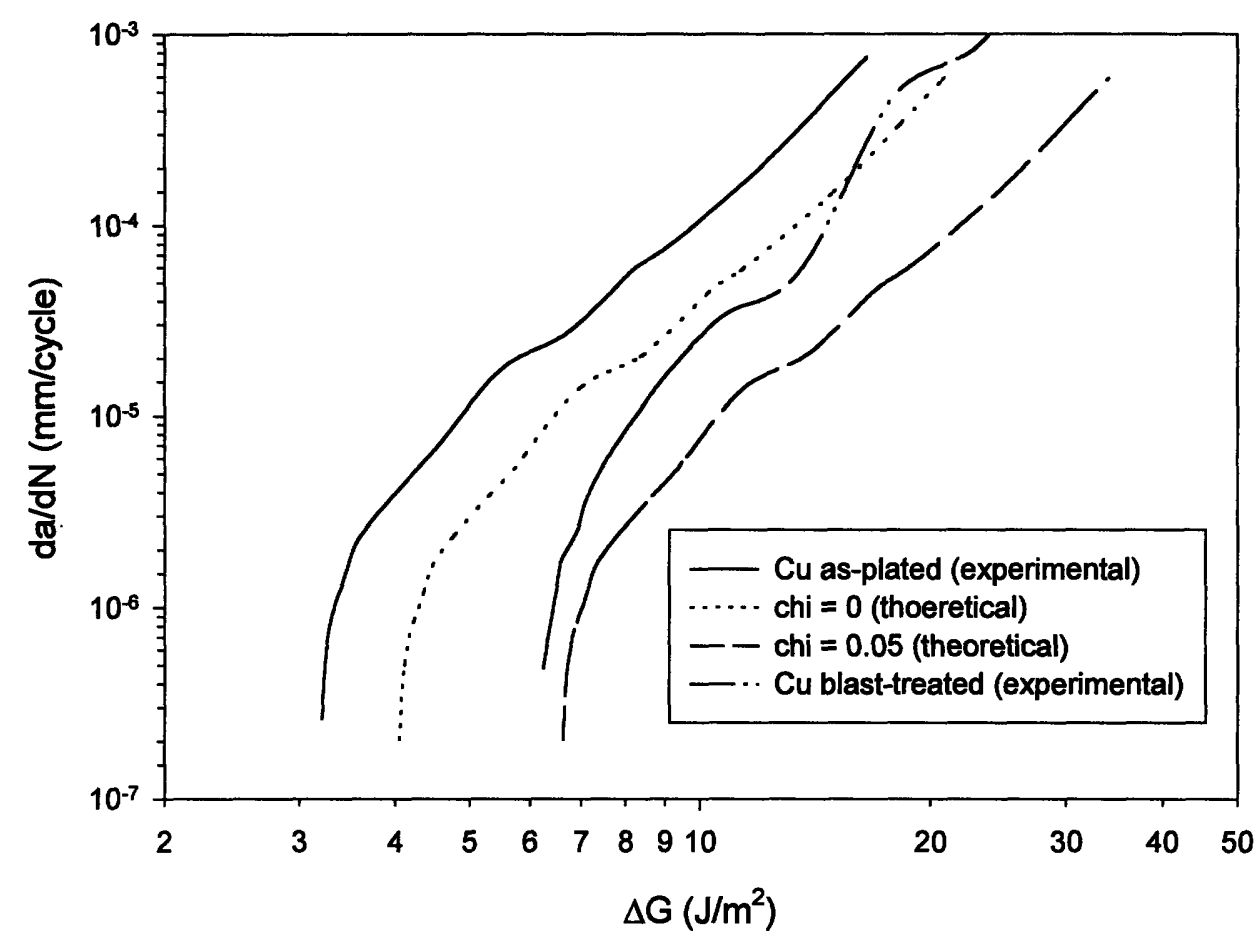

Figure 4.19 - Predicted FCP rates for deflected cracks as functions of the closure factor $\chi$ for $\theta=45^{\circ}$ and $D / D+S=0.75$ along with data for the as-plated and blast-treated copper-encapsulant interfaces

\subsubsection{Roughness-induced Closure}

Shielding due to crack deflection is active in all growth regimes. This has the effect of shifting the entire crack growth curve to higher levels of $\Delta G$, which is consistent with the data for material B on as-plated vs. blast-treated nickel. However, the data for the asplated vs. blast-treated copper specimens show a greater difference in FCP resistance near threshold, but less difference at higher growth rates. Therefore, another shielding mechanism which is more dominant at lower growth rates must also be present.

One possible mechanism to explain enhanced crack-tip shielding at low growth rates is roughness-induced closure. In a study of the effects of non-planarity on 
interfacial fracture resistance, Evans and Hutchinson [61] noted that frictional contact and locking of crack surface facets could shield the crack tip and modify the energy release rate. Figure 4.20 shows three possible conditions for a crack along a faceted interface. At large values of crack growth driving force, the crack will be open everywhere, and closure will not occur. However, as the driving force decreases, the crack opening displacement becomes smaller, and contact and crack face sliding become more likely. Under certain conditions, frictional locking can occur, preventing further propagation of the crack tip [61].

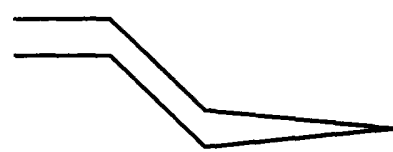

a

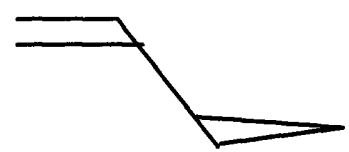

b

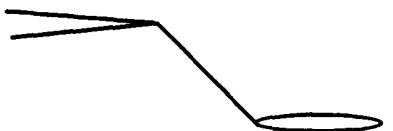

c

Figure 4.20 - Three conditions for a crack along a faceted interface:

a) open everywhere, b) contact with sliding, c) frictional locking

Neglecting friction, Evans and Hutchinson developed a contact zone model for predicting the extent of crack shielding for an idealized, undulating interface [61]. They determined that the contact zone was determined by a material parameter, $\zeta$, given by:

$$
\zeta=\frac{E H}{G_{0}}
$$

where $E$ is the modulus of the substrate material, $H$ is the undulation height, and $G_{0}$ is the intrinsic interface toughness. The transition from no contact to full contact was found to occur over a relatively small range of $\zeta$ between $\sim 10^{-2}$ (no contact) and $\sim 10$ (full contact). It is interesting to note that the difference in root mean square peak height, $R_{q}$ (defined in section 3.2.2), between as-plated copper and blast-treated copper is approximately an order of magnitude. Thus if $R_{q}$ is taken to be approximately equivalent to $H$, then an estimate of $\zeta$ can be made. For the interfaces studied, $\zeta$ ranged from approximately 80 (for the as-plated copper specimen) to 800 (for the blast-treated copper specimen). These 
values fell into the regime, defined by Evans and Hutchinson, where roughness-induced shielding and full contact are expected to occur. Furthermore, the extent of closure and shielding are expected to increase with $\zeta$. These roughness-induced closure trends are observed in the near-threshold FCP data.

\subsubsection{Asperity Bridging}

Fractography of the encapsulant fatigue-fracture surface of material A bonded to as-plated nickel revealed metal particles embedded in the polymer. Thus, it is possible that as the crack front approached and advanced past these embedded asperities, the asperities could have bridged the crack tip, much like fiber bridging in fiber-reinforced composites.

Figure 4.21 illustrates the hypothesized asperity-bridging mechanism. In effect, the metal asperity could have bridged the crack, remaining intact even after the crack tip had passed. Eventually, the asperity would deform to the point of fracture and break. The plastic deformation and subsequent fracture of the metal would absorb much energy during the crack propagation process, acting as a toughening mechanism. Fiber bridging in fiber-reinforced composites has been found to shift the FCP curve to the right towards higher driving forces, and decrease the slope of the curve in the Paris regime [39]. A similar trend is seen when the FCP curve of material A (asperity bridging) is compared to material B (no asperity bridging) in Figure 4.8. 

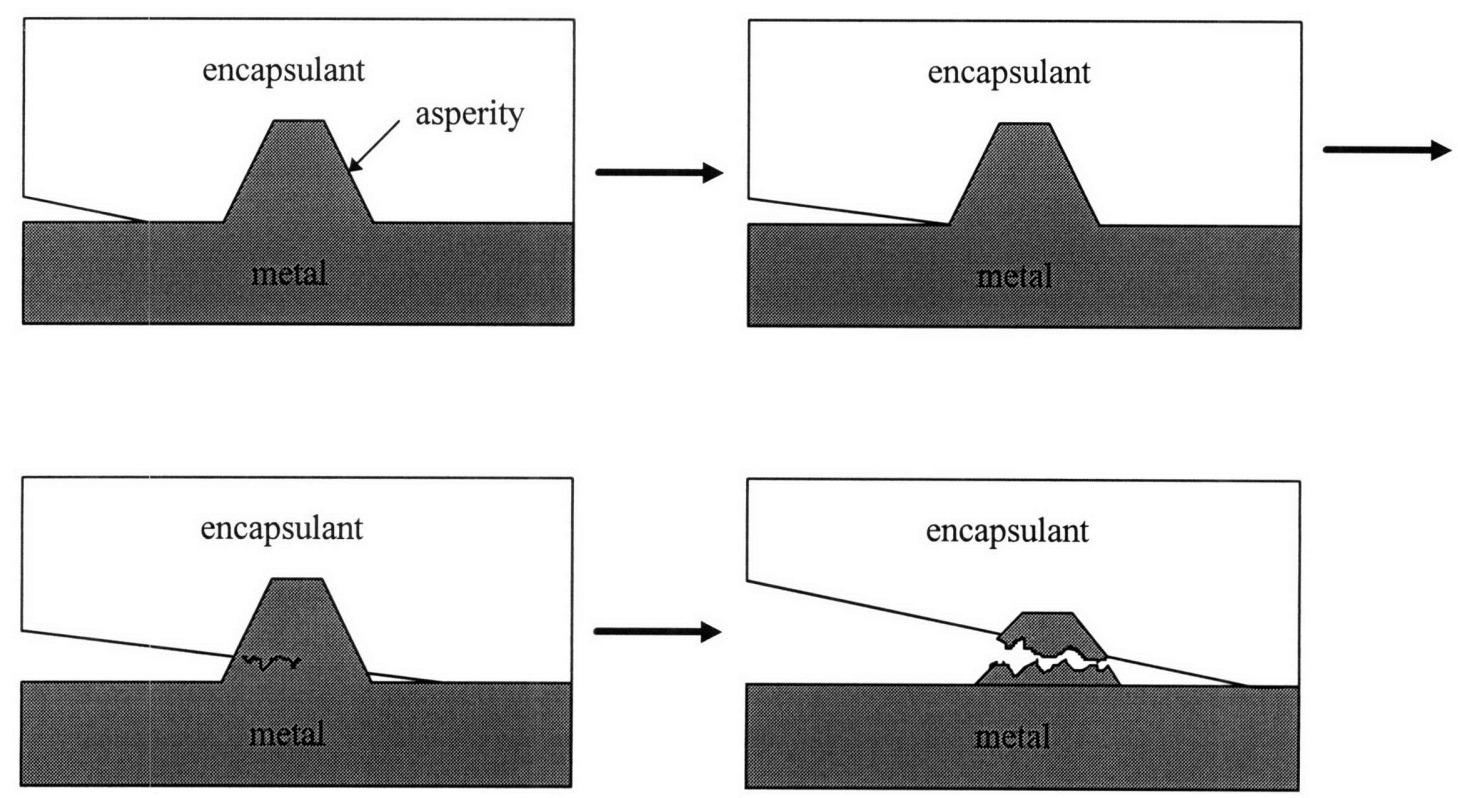

Figure 4.21 - Illustration of the proposed asperity bridging process 


\section{Chapter 5}

\section{Conclusions}

\subsection{Contact Angle Goniometry}

The three-liquid probe method was evaluated for probing the surface energy of metals and filled polymers and determining the thermodynamic work of adhesion for metal/polymer adhesive systems. Based on this work, the following conclusions can be made:

- Scatter in the contact angle measurements made discrimination between the various metallic surfaces difficult. Discrimination between the polymer encapsulant surfaces was much better, but there were doubts as to whether the encapsulant surface cured against air was representative of the surface when bonded to metal.

- Scatter in the contact angle measurements for the rougher metallic surfaces indicated that the high surface roughness could have increased contact angle measurements, thereby influencing subsequent calculations of surface energy.

- The thermodynamic work of adhesion of encapsulant/metal systems as calculated from the components of surface energy did not correlate to package level delamination results, and is therefore not a reliable predictor of interfacial adhesion.

\subsection{Interfacial Fatigue Crack Propagation Testing}

A test method was developed to study subcritical crack propagation along polymer/metal interfaces using nominally Mode I double cantilever beam sandwich test specimens. The results of this experimental work led to the following conclusions: 
- Cyclic crack growth rate vs. strain energy release rate range ( $d a / d N$ vs. $\Delta G$ ) data were collected for fatigue crack propagation along polymer/metal interfaces. The crack growth rate was found to have a power-law dependence on $\Delta G$, in agreement with relationships found for bulk fatigue crack propagation in metals, ceramics, and polymers.

- Fatigue crack propagation was found to occur at $G$ levels orders of magnitude below typical mode I fracture energies for polymer/metal interfaces. The polymer/metal interfaces were also found to exhibit a fatigue crack growth threshold $\left(\Delta G_{t h}\right)$.

- The experimental technique was able to discriminate between three encapsulant materials bonded to nickel, and the FCP resistance of each system correlated to levels of interfacial delamination observed after package-level reliability testing. Thus, interfacial FCP resistance was shown to be a good indicator of material performance in microelectronic packaging applications.

- By testing specimens made from substrates with different plating metals and different levels of macroscopic roughness, it was shown that interfacial FCP resistance increased with substrate surface roughness, both at threshold, and at higher crack growth rates.

- Interfacial FCP resistance was not correlated to the work of adhesion as calculated from contact angle measurements. This was due to either: 1) error in the contact angle measurements or incorrect methodology for calculating $W^{a}$, or 2) the other energy dissipating processes that occurred during FCP obscured the effect of $W^{a}$.

\subsection{Interfacial Crack-tip Shielding Mechanisms}

Based on the collected fatigue crack propagation data and subsequent fractography, three interfacial crack-tip shielding mechanisms were proposed:

- Crack deflection along the interface provided crack-tip shielding in all regimes of crack growth. This was supported by the data showing increasing FCP resistance with interface roughness.

- Roughness-induced closure was another source of crack-tip shielding, especially at low growth rates when crack opening displacements were small. This was supported 
by the data showing enhanced FCP resistance at low growth rates for the encapsulant/copper interfaces, where the difference in interface roughness is greatest between the as-plated and blast-treated specimens.

- A crack-tip shielding mechanism involving crack-bridging by metal asperities was proposed to explain the enhanced FCP resistance of material A on as-plated nickel. This notion was supported by the presence of metal particles embedded in the encapsulant fatigue-fracture surface.

\subsection{Recommendations for Future Work}

Although the three-liquid probe contact angle method did not prove to be useful in this study, it may still have applications. Because surface roughness influences the contact angle, the technique should probably be restricted to the study of very smooth surfaces. Since it is so sensitive to the properties of the surface, it would be useful for detecting contamination, comparing cleaning processes, etc. The technique is not recommended for calculating the work of adhesion of polymer/metal systems for the purpose of predicting in-service delamination.

Interfacial fatigue crack propagation testing was shown to be useful for predicting delamination at the encapsulant/heat slug interface. The groundwork has been established for studying other interfaces of interest. The next logical step is to characterize other polymer-metal interfaces identified as needing materials development. Plans are in place for this to be the next phase of this research program.

Besides thermomechanical stresses, microelectronic package interfaces must also withstand moisture-related stressing. Polymer/metal interfacial adhesion is known to degrade when exposed to very humid environments [1]. Therefore, the fatigue crack propagation resistance of the interface in the presence of moisture would be of great interest. The results from such an experiment could be used to determine the extent of environmentally assisted cracking versus cracking due to mechanical loading. One would also expect the mechanism of crack propagation to change under such conditions.

All of the experiments in this study were conducted under nominally mode I loading conditions. However, studies of interfacial fracture often involve testing over a 
range of mode mixities. The test method presented here could easily be modified to determine the mixed-mode FCP characteristics of polymer metal interfaces by varying the ratio of the heights of the two substrate beams [62]. In fact, the results of some experiments dealing with mixed-mode FCP along epoxy/aluminum interfaces have recently been reported [63]. Testing over a range of mode mixities could help elucidate crack propagation mechanisms and provide for more accurate simulations of actual loading conditions. 


\section{Appendix}

Figure A.1 shows the percent error between $\Delta G$ calculations using the homogenous solution (equation 3.11) and the exact solution (equation 3.12) as a function of dimensionless crack length $(a / W)$. The homogeneous solution consistently overestimated the strain energy release rate range. Furthermore, the magnitude of the error was larger for short crack lengths and diminished as $a / W$ increased. For all of the data collected in this study, the error was $\sim 2-4 \%$, so the choice of using the homogeneous or exact solution was not critical. However, if the thickness of the adhesive layer were increased, one would expect the error to increase, which would necessitate using the exact solution.

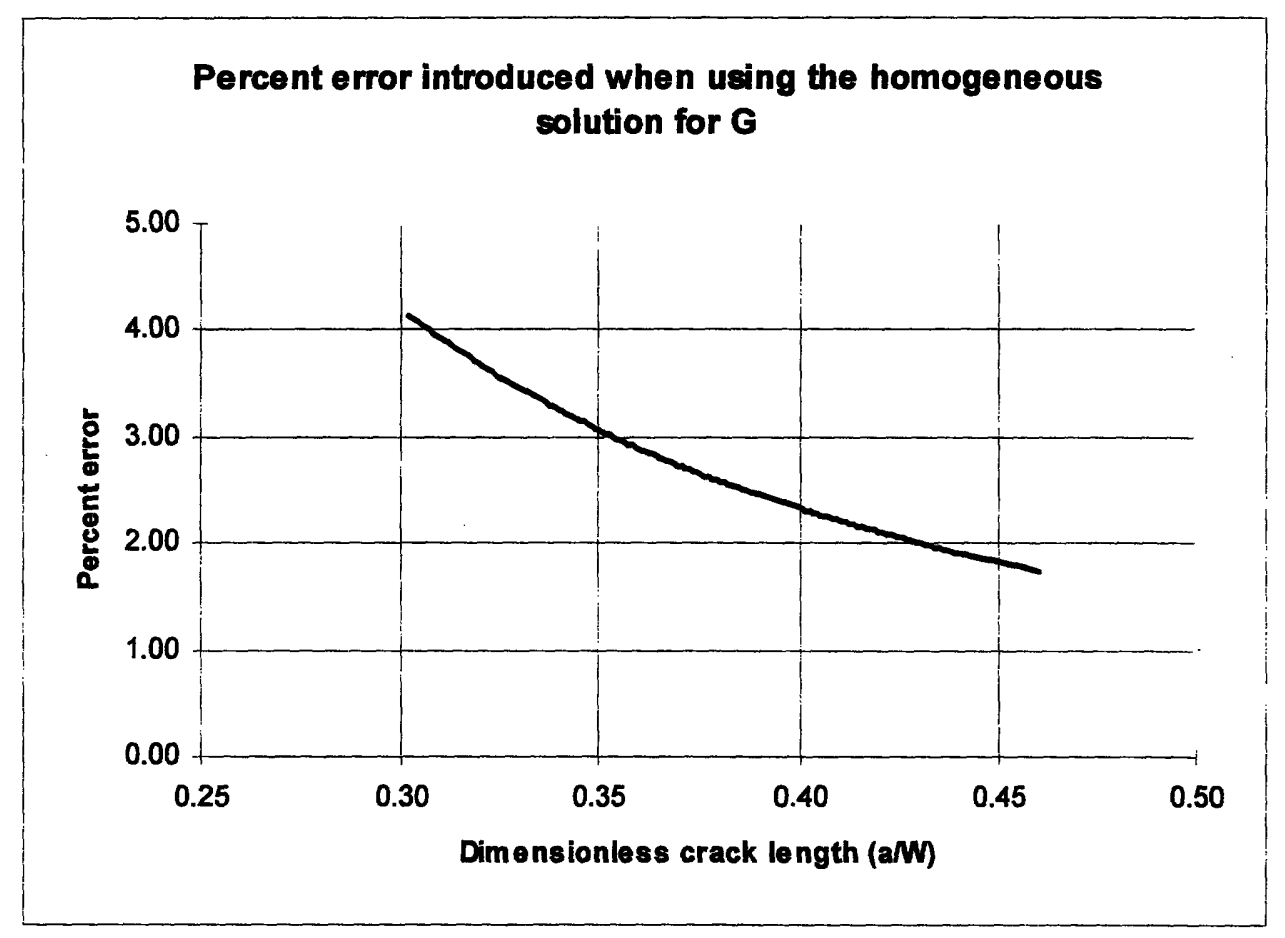

Figure A.1 - Percent error between $\Delta G$ calculations using the homogenous solution (equation 3.11) and the exact solution (equation 3.12) as a function of dimensionless crack length $(a / W)$ 


\section{Bibliography}

[1] Kinloch, A.J, Adhesion and Adhesives - Science and Technology, Chapman and Hall, 1987.

[2] R.D. Adams, "Failure Strength Tests and Their Limitations," Engineering Materials Handbook, Vol. 2; Adhesives and Sealants, ASM International, Metals Park, OH, pp. 325-334.

[3] Azimi, H.A. and Wakharkar V., Intel Internal Report, 1995.

[4] Bradendburger, P.D., Mixed-Mode Fracture of Organic Chip Attachment Adhesives, MS Thesis, Lehigh University, Bethlehem, PA, 1995.

[5] S. Liu, Y. Mei, and T.Y. Wu, IEEE Transactions on Components, Packaging and Manufacturing Technology - Part A, Vol. 18, No. 3, 1995, pp. 618-625.

[6] S. Mall and K.T. Yun, J. Adhesion, Vol. 23, 1987, pp. 215-231.

[7] S. Mall and W.S. Johnson in "Debonding Characteristics of Adhesively Bonded Woven Kevlar Composites," Adhesively Bonded Joints: Testing, Analysis, and Design, ASTM STP 981, W.S. Johnson, Ed. ASTM, Philadelphia, 1988, pp. 194-206.

[8] S. Mall and W.S. Johnson in "Characterization of Mode I and Mixed-mode Failure of Adhesive Bonds Between Composite Adherends," Composite Materials: Testing and Design (Seventh Conference), ASTM STP 893, J.M. Whitney, Ed., ASTM, Philadelphia, 1986, pp. 322-334.

[9] D.A. Jablonski, J. Adhesion, Vol. 11, 1980, pp. 124-133.

[10] R. Joseph, et. al. J. Adhesion, Vol. 41, 1993, pp. 169-187.

[11] A.J. Kinloch and S.O. Osiyemi, J. Adhesion, Vol. 43, 1993, pp. 79-90.

[12] D.W. Schmuesser, J. Adhesion, Vol. 36, 1991, pp. 1-23.

[13] T.B. Llyod, Colloids and Surfaces A, Vol. 93, 1994, pp. 25-37.

[14] R.J. Good, J. Adhesion Sci. Technol., Vol. 6, 1992, pp. 1269-1302.

[15] C.J. Van Oss, et al., Chem. Rev., Vol. 88., 1988, pp. 927.

[16] F.M. Fowkes, Surface and Interfacial Aspects of Biomedical Polymers, J.D. Andrade, Ed., Plenum , NY, 1985. 
[17] A.N. Gent and J. Shultz, Journal of Adhesion, Vol. 3, 1972, pp. 281.

[18] E.H. Andrews and A.J. Kinloch, Proc. R. Soc. London, Series A, Vol. 332, 1973, pp. 385-401.

[19] A.A. Griffith, Phil. Trans. Roy. Soc. London, Series A, Vol. A221, 1920, pp. 163.

[20] G.R. Irwin, Fracturing of Metals, ASM, Cleveland, OH, 1948, pp. 147.

[21] E. Orowan, Fatigue and Fracture of Metals, MIT Press, Cambridge, MA, 1950, pp. 139.

[22] R.S. Rivlin and A.G. Thomas, J. Polymer Sci., Vol. 10, 1953, pp. 291.

[23] J.P. Berry, J. Polymer Sci., Vol. 50, 1961, pp. 107.

[24] T.L. Anderson, Fracture Mechanics, CRC Press, Boca Raton, FL, 1995.

[25] F. Xiao, et al. J. Mat. Sci., Vol. 28, 1993, pp. 5620-5629.

[26] M. Charalambides, A.J. Kinloch, Y. Wang, and J.G. Williams, International Journal of Fracture, Vol. 54, 1992, pp. 269-291.

[27] M.L. Williams, Bull. Seismol. Soc. America, Vol. 49, 1959, pp. 199-204.

[28] A.H. England, Journal of Applied Mechanics, Vol. 44, 1965, pp. 400-402.

[29] F. Erdogan, Journal of Applied Mechanics, Vol. 44, 1965, pp. 403-410.

[30] J.R. Rice, Journal of Applied Mechanics, Vol. 55, 1988, pp. 98-103.

[31] Mostovoy, et al., Journal of Materials, Vol. 2, 1967, pp. 661-681.

[32] A.G. Evans and B.J. Dalgleish, Materials Science and Engineering, A162, 1993, pp. $1-13$.

[33] F. Ozdil and L.A. Carlsson, Engineering Fracture Mechanics, Vol. 41, 1992, pp. 475-485.

[34] F. Gaudette, Ph.D. Thesis in progress, M.I.T., Cambridge, MA, 1996.

[35] P.C. Paris, M.P. Gomez, and W.E. Anderson, The Trend in Engineering, Vol. 13, 1961, p. 9. 
[36] P.C. Paris and F. Erdogan, J. Basic Eng. Trans., ASME, Series D, Vol. 85, 1963, p. 528

[37] R.O. Ritchie, Mat: Sci. and Eng., A103, Vol. 15, 1988.

[38] S. Suresh, Fatigue of Materials, Cambridge University Press, 1991.

[39] K.S. Chan and D.L. Davidson, Proc. of the Eng. Foundation, Int. Conf., Santa Barbara, CA 1991, p.325.

[40] S. Mostovoy and E. J. Ripling, in Adhesion Science and Technology, L.H. Lee, Ed., (Plenum Press, New York, 1975, p. 514.

[41] D.A. Jablonski, J. Adhesion, Vol. 11, 1980, pp. 124-133.

[42] S. Mall and W.S. Johnson in "Characterization of Mode I and Mixed-mode Failure of Adhesive Bonds Between Composite Adherends," Composite Materials: Testing and Design (Seventh Conference), ASTM STP 893, J.M. Whitney, Ed., ASTM, Philadelphia, 1986, pp.322-334.

[43] S. Mall and K.T. Yun, J. Adhesion, Vol. 23, 1987, pp. 215-231.

[44] S. Mall and W.S. Johnson in "Debonding Characteristics of Adhesively Bonded Woven Kevlar Composites," Adhesively Bonded Joints: Testing, Analysis, and Design, ASTM STP 981, W.S. Johnson, Ed. ASTM, Philadelphia, 1988, pp. 194-206.

[45] C. Lin and K.M. Liechti, J. Adhesion, Vol. 21, 1987, pp. 1-24.

[46] S. Mall and K.T. Yun, J. Adhesion, Vol. 23, 1987, pp. 215-231.

[47] D. W. Schmusser, J. Adhesion, Vol. 36, 1991, pp. 1-23.

[48] R. Joseph, J.P. Bell, A.J. McEvily, J.L. Liang, J. Adhesion, Vol. 41, 1993, pp. 169187.

[49] A.J. Kinloch and S.O. Osiyemi, J. Adhesion, Vol. 43, 1993, pp. 79-90.

[50] R.M. Cannon, B.J. Dalgleish, R.H. Dauskardt, T.S. Oh, and R.O.Ritchie, Acta Metalla. Mater., Vol. 39, 1991, pp. 2145-2156.

[51] J.E. Ritter, W. Grayeski, and T.J. Lardner, "Crack Propagation in Polymer/Glass Interfaces Under Monotonic and Cyclic Loading," presented at the 1995 ASME International Mechanical Engineering Congress and Exposition, San Francisco, CA, Nov. 12-17, 1995. 
[52] J.E. Ritter, to be published in Application of Fracture Mechanics in Electronic Packaging and Materials, ASME, 1996.

[53] D. Yao, Z. Zhang, and J.K. Shang, "Experimental Technique for Studying Mixedmode Crack Growth in Solder Joints," presented at the 1995 ASME International Mechanical Engineering Congress and Exposition, San Francisco, CA, Nov. 12-17, 1995.

[54] D. Yao and J.K. Shang, "Effect of Load-mix on Fatigue Crack Growth in Sn-Pb Solder Joint," presented at the 1995 ASME International Mechanical Engineering Congress and Exposition, San Francisco, CA, Nov. 12-17, 1995.

[55] J.K. Shang and D. Yao, "Effect of Interface Roughness on Fatigue Crack Growth in $\mathrm{Sn}-\mathrm{Pb}$ Solder Joint," presented at the 1995 ASME International Mechanical Engineering Congress and Exposition, San Francisco, CA, Nov. 12-17, 1995.

[56] R.M.L. Foote and V.T. Buchwald, International Journal of Fracture, Vol. 29, 1985, pp. 125-134.

[57] Annual Book of ASTM Standards, American Society for Testing and Materials, Philadelphia, PA, 1990, p. 226.

[58] R.N. Wenzel, Ind. Eng. Chem., Vol. 28, 1936, p. 988.

[59] K. Van, Effect of IC Mold Compound Formulation Variables on Package Interfacial Adhesion, MS Thesis, M.I.T., Cambridge, MA, 1992.

[60] S. Suresh, Metallurgical Transactions A, Vol. 16A, 1985, pp. 249-260.

[61] A.G. Evans and J.W. Hutchinson, Acta Metall., Vol. 37, 1989, pp. 909-916.

[62] J.W. Smith, et al., J. Mat. Sci., Vol. 28, 1993, pp. 4234-4244.

[63] J.K. Shang, "Interface Fatigue-Crack Growth in Layered Materials," Proceedings of the Sixth International Fatigue Congress, Berlin, Germany, May 6-10, 1996, pp. 43-54. 